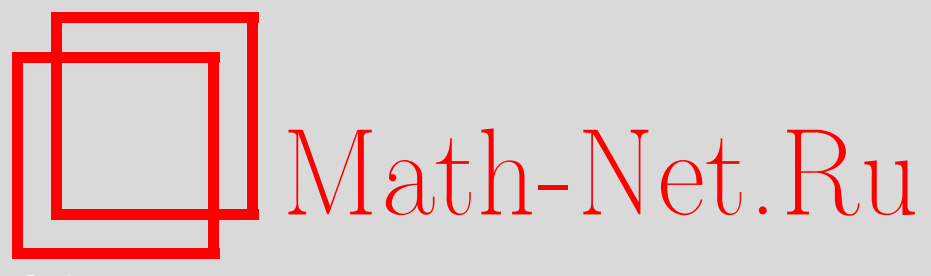

Е. А. Копылова, Асимптотическая устойчивость солитонов для нелинейных гиперболических уравнений, $У \mathrm{MH}$, 2013, том 68, выпуск 2, 91-144

DOI: https://doi.org/10.4213/rm9509

Использование Общероссийского математического портала Math-Net.Ru подразумевает, что вы прочитали и согласны с пользовательским соглашением http://www . mathnet.ru/rus/agreement

Параметры загрузки:

IP : 52.87 .193 .239

26 апреля 2023 г., 16:38:25

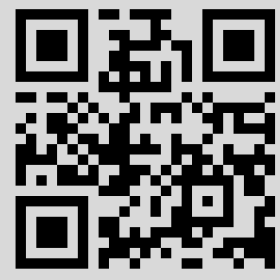




\title{
Асимптотическая устойчивость солитонов для нелинейных гиперболических уравнений
}

\author{
Е. А. Копылова
}

Дается обзор основных результатов по асимптотической устойчивости солитонов, а также методов ее доказательства на примере нелинейного релятивистского волнового уравнения с потенциалом Гинзбурга-Ландау. Асимптотическая устойчивость означает, что решение уравнения с начальными данными, близкими к одному из солитонов, при больших временах асимптотически представляет собой сумму некоторого, возможно другого, солитона и дисперсионной волны, являющейся решением соответствующего линейного уравнения. Методы доказательства основаны на спектральных свойствах линеаризованного уравнения и представляют собой современное развитие теории устойчивости Ляпунова. Также построены примеры нелинейных уравнений с заданными спектральными свойствами линеаризованной динамики.

Библиография: 45 названий.

Ключевые слова: нелинейные гиперболические уравнения, асимптотическая устойчивость, релятивистская инвариантность, гамильтонова структура, симплектическая проекция, инвариантное многообразие, солитон, кинк, золотое правило Ферми, рассеяние солитонов, асимптотическое состояние.

DOI: $10.4213 / \mathrm{rm} 9509$

1. Введение................................................ 93

1.1. Обзор литературы................................. 93

1.2. Постановка задачи ................................. 94

1.3. Результаты ........................................ 96

1.4. Методы............................................ 97

1.5. Открытые проблемы .................................. 98

1.6. О распределении материала ......................... 99

Глава І. Бегущие солитоны . . . . . . . . . . . . . . . . . . . . . . . . . . 99

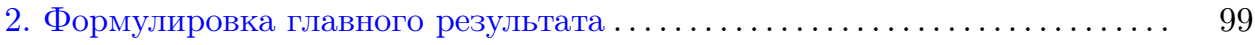

Работа выполнена при поддержке РФФИ (грант № 12-01-00203-а) и Austrian Science Fund (FWF): M1329-N13. 


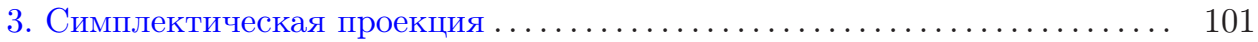

3.1. Симплектическая структура и гамильтонова форма........... 101

3.2. Симплектическая проекция на солитонное многообразие....... 101

4. Линеаризация на солитонном многообразии . . . . . . . . . . . . . . . 102

4.1. Гамильтонова структура и спектр ..................... 104

4.2. Убывание трансверсальной линеаризованной динамики ....... 106

4.3. Оценки нелинейного члена . . . . . . . . . . . . . . . . . . . . 107

5. Симплектическое разбиение динамики ...................... 108

6. Модуляционные уравнения . . . . . . . . . . . . . . . . . . . . 109

7. Убывание трансверсальной динамики ..................... 110

7.1. Замороженная трансверсальная динамика................ 111

7.2. Интегральные неравенства.......................... 113

7.3. Симплектическая ортогональность ................... 114

7.4. Убывание трансверсальной компоненты ................ 115

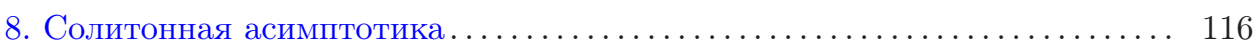

Глава II. Стоячий солитон . . . . . . . . . . . . . . . . . . . . . . 118

9. Формулировка главного результата ....................... 118

10. Линеаризация на солитоне . . . . . . . . . . . . . . . . . . . . . . 118

11. Асимптотическое разложение динамики ................... 120

11.1. Асимптотическое разложение $\dot{z} \ldots \ldots \ldots \ldots \ldots \ldots \ldots \ldots \ldots \ldots \ldots$

11.2. Асимптотическое разложение $\dot{f} \ldots \ldots \ldots \ldots \ldots \ldots \ldots \ldots \ldots . \ldots \ldots . \ldots \ldots$

12. Нормальные формы Пуанкаре .......................... 121

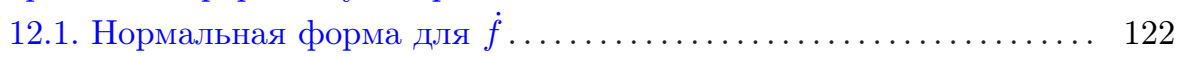

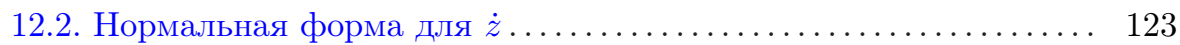

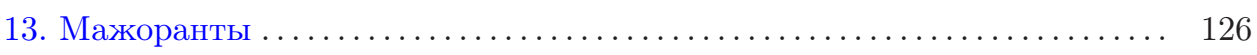

13.1. Начальные условия и оценка для $g \ldots \ldots \ldots \ldots \ldots \ldots \ldots \ldots \ldots . \ldots \ldots$

13.2. Система мажорант ............................ 126

13.3. Оценки остаточных членов ....................... 126

13.4. Оценки для мажорант ............................ 128

13.5. Равномерные оценки для мажорант .................... 129

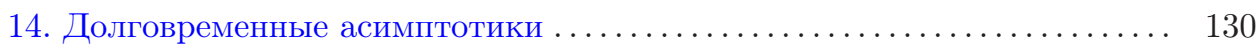

14.1. Долговременное поведение $z(t) \ldots \ldots \ldots \ldots \ldots \ldots \ldots \ldots \ldots \ldots . \ldots \ldots$

14.2. Солитонная асимптотика ....................... 131

Глава III. Примеры нелинейных потенциалов . . . . . . . . . . . . . . . . 134

15. Кусочно параболические потенциалы . . . . . . . . . . . . . . . . 134

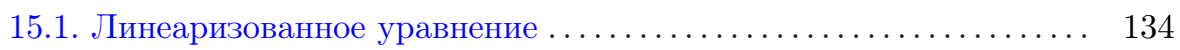

15.2. Нечетные собственные функции . . . . . . . . . . . . . . . . . . 135

15.3. Четные собственные функции . . . . . . . . . . . . . . . . 137

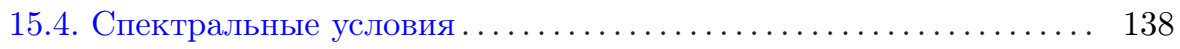

16. Гладкие аппроксимации . . . . . . . . . . . . . . . . . . . . . 140

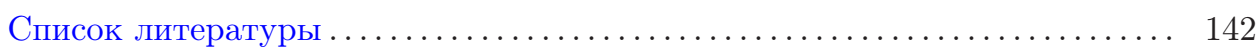




\section{1. Введение}

В последнее десятилетие бурное развитие получила теория асимптотической устойчивости солитонов для нерелятивистских нелинейных уравнений. Солитонным решениям принадлежит особая роль при изучении эволюционных уравнений ввиду того, что зачастую они довольно легко находятся численно и, кроме того, возникают, как правило, при изучении долговременного поведения решений этих уравнений. Первые результаты в этом направлении получили в 1965 г. Н. Забуский и М. Крускал для уравнения KdV в результате численного моделирования [45]. В 1967 г. К. Гарднер, Д. Грин, М. Крускал и Р. Миура обнаружили, что обратное преобразование рассеяния позволяет решить уравнение $\mathrm{KdV}$ аналитически [12]. Выяснилось, что любое решение этого уравнения с достаточно гладкими быстроубывающими начальными данными сходится к конечной сумме солитонов, движущихся направо, и дисперсионной волны, движущейся налево. Полный обзор этих исследований можно найти в [10]. Эти результаты были распространены на другие интегрируемые уравнения в работах А. Итса, Е. Я. Хруслова, А. Б. Шабата, В. Е. Захарова и других (см. [11]).

Исследование асимптотической устойчивости солитонов инспирировано проблемой устойчивости и эффективной динамики элементарных частиц, поскольку они могут быть отождествлены с солитонами нелинейных полевых уравнений. Такое отождествление соответствует духу теории элементарных частиц в контексте нелинейных гиперболических уравнений в частных производных, предложенной В. Гейзенбергом [13], [14].

Недавние численные эксперименты [22] показывают, что решения общих неинтегрируемых нелинейных волновых уравнений с начальными данными конечной энергии при больших временах распадаются на конечное число слабо взаимодействующих солитонов и убывающую дисперсионную волну. Данная работа посвящена методам доказательства подобных асимптотик с одним солитоном для неинтегрируемых уравнений при начальных данных, близких к солитонному многообразию.

1.1. Обзор литературы. Первоначально асимптотическая устойчивость солитонов была доказана А. Соффером и М. Вайнштейном [38], [39], [44] (см. также [34]) для нелинейного $U(1)$-инвариантного уравнения Шрёдингера с потенциалом для малых начальных данных и малого коэффициента при нелинейном члене.

Позднее в работах В. С. Буслаева и Г. С. Перельман [5], [6] этот результат был установлен для более трудного случая, а именно для трансляционно инвариантного, $U(1)$-инвариантного нелинейного одномерного уравнения Шрёдингера в случае, когда линеаризованная на солитоне динамика не имеет ненулевых собственных значений. Техника, аналогичная [5], [6], была развита Ю. С. Миллер, Р. Пего и М. Вайнштейном для одномерных модифицированных $\mathrm{KdV}$ - и RLW-уравнений [30], [32].

Одномерное нелинейное уравнение Шрёдингера с более сложным ненулевым дискретным спектром линеаризованной динамики было рассмотрено В. С. Буслаевым и К. Сулем [7] (см. также [43]). Другие размерности рассмотрены в работах [8], [17], [37], [42]. 
Асимптотическая устойчивость солитонов для трехмерного нелинейного уравнения Клейна-Гордона с потенциалом была доказана в работе [40] и для систем типа поле-частица - в работах [15], [16].

В работе С. Куканьи [9] установлена асимптотическая устойчивость волнового фронта для трехмерного релятивистского волнового уравнения. Волновым фронтом называется решение, зависящее только от одной пространственной переменной. Такое решение не является солитоном, так как оно имеет бесконечную энергию.

Асимптотическая устойчивость стоячих солитонов для уравнения Дирака с потенциалом в трехмерном случае была установлена Н. Буссаидом [2] и для уравнения Дирака без потенциала - Н. Буссаидом и С. Куканьей в [3]. Одномерный случай рассмотрен Д. Пелиновским и А. Стефановым [33].

Асимптотической устойчивости солитонов посвящены также наши работы [4], [18], [20], [21], [23], [25]. Асимптотическая устойчивость солитонов (кинков) для релятивистского нелинейного волнового уравнения Гинзбурга-Ландау впервые установлена в недавних совместных работах автора и А.И. Комеча [27], [28]. Основная стратегия доказательства асимптотической устойчивости является общей для всех вышеперечисленных работ. Однако ее реализация для релятивистского случая столкнулась с рядом трудностей, что препятствовало развитию теории в течение двадцати лет.

В данной статье представлена общая стратегия работ [5]-[7], [16], а также новые методы, разработанные в [27], [28].

1.2. Постановка задачи. Основная часть работы посвящена одномерному нелинейному волновому уравнению

$$
\ddot{\psi}(x, t)=\psi^{\prime \prime}(x, t)+F(\psi(x, t)), \quad x \in \mathbb{R} .
$$

Мы предполагаем, что $F(\psi)=-U^{\prime}(\psi)$, где $U(\psi)$ - потенциал типа Гинзбурга-Ландау, т. е. $U(\psi)$ удовлетворяет следующим условиям (см. рис. 1$)$.

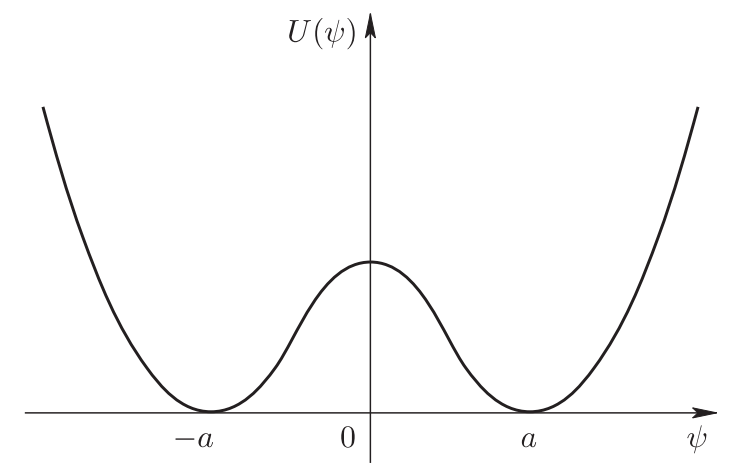

Рис. 1. Потенциал $U(\psi)$

U1. Потенциал $U(\psi)$ является гладкой четной функцией такой, что

$$
U(\psi)>0 \quad \text { npu } \psi \neq a .
$$




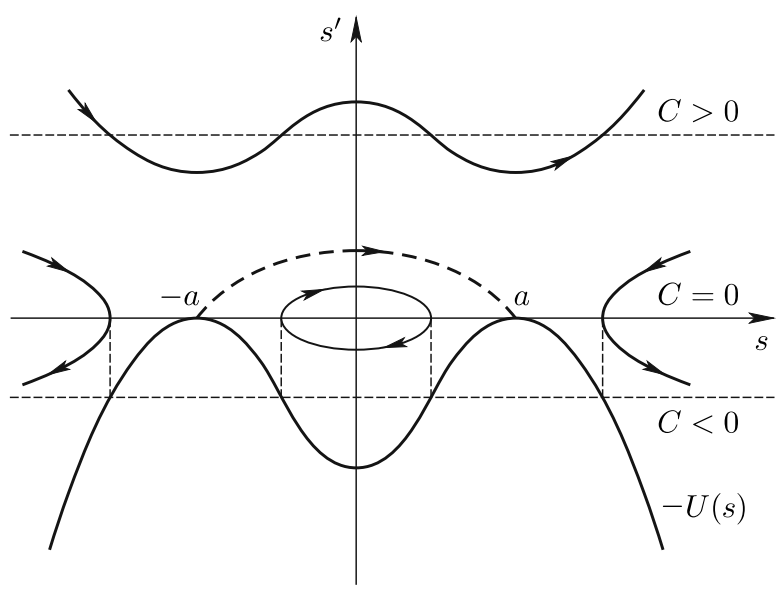

Рис. 2. Фазовый портрет

U2. В окрестности точек \pm а потенииал является параболой:

$$
U(\psi)=\frac{m^{2}}{2}(\psi \mp a)^{2}, \quad|\psi \mp a|<\delta,
$$

с некоторыми $0<\delta<a / 2$ u $m>0$.

Соответствующее стационарное уравнение имеет вид

$$
s^{\prime \prime}(x)-U^{\prime}(s(x))=0, \quad x \in \mathbb{R} .
$$

Это уравнение имеет постоянные решения $\psi(x) \equiv 0$ и $\psi(x) \equiv \pm a$. Не постоянные решения найдем при помощи "интеграла энергии":

$$
\frac{\left(s^{\prime}\right)^{2}}{2}-U(s)=C,
$$

где $C$ - произвольная постоянная. На рис. 2 изображен фазовый портрет данного уравнения. Мы видим, что при $C=0$ существует так называемый кинк непостоянное решение $s(x)$ стационарного уравнения (1.4), обладающее конечной энергией и удовлетворяющее условию $s(x) \rightarrow \pm a$ при $x \rightarrow \pm \infty$ (см. рис. 3 ). Кроме того, из условия U2 следует, что $(s(x) \mp a)^{\prime \prime}=m^{2}(s(x) \mp a)$ при $x \rightarrow \infty$. Поэтому

$$
|s(x) \mp a|=C e^{-m|x|}, \quad x \rightarrow \pm \infty,
$$

т. е. кинк приближается к своим асимптотам $\pm a$ с экспоненциальной скоростью. Так как уравнение (1.1) является релятивистски инвариантным, то движущиеся со скоростью $|v|<1$ солитоны (или кинки) $s_{q, v}(x, t)=s(\gamma(x-v t-q))$, $q \in \mathbb{R}$, также являются решениями уравнения (1.1). Здесь $\gamma=1 / \sqrt{1-v^{2}}-$ лоренцево сокращение. Линеаризуем уравнение (1.1) на кинке $s(x)$. Подставляя разложение $\psi(x, t)=s(x)+\phi(x, t)$ в это уравнение, формально получим

$$
\ddot{\phi}(x, t)=-H \phi(x, t)+\mathscr{O}\left(|\phi(x, t)|^{2}\right),
$$




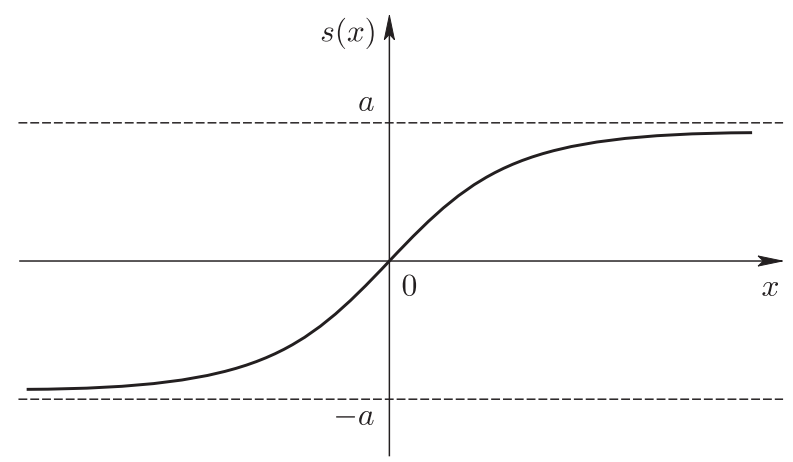

Рис. 3. Кинк

где $H:=-d^{2} / d x^{2}+m^{2}+V(x)$ - оператор Шрёдингера с потенциалом $V(x)=$ $-F^{\prime}(s(x))-m^{2}=U^{\prime \prime}(s(x))-m^{2}$. Легко проверить, что оператор $H$ обладает следующими свойствами.

H1. Непрерывный спектр оператора $H$ совпадает с интервалом $\left[m^{2}, \infty\right)$.

H2. Точка $\lambda_{0}=0$ является точкой дискретного спектра с собственной функцией $s^{\prime}(x)$.

H3. Так как $s^{\prime}(x)>0$, то $\lambda_{0}$ является основным состоянием, а все остальные точки дискретного спектра, если они существуют, содержатся в интервале $\left(0, m^{2}\right]$.

Предположим дополнительно, что выполнено следующее условие.

U3. Концевая точка $\lambda=m^{2}$ непрерывного спектра оператора $H$ не является ни собственным значением, ни резонансом.

ЗАмечание 1.1. Точное определение резонанса в одномерном случае можно найти в работе [31] (см. также [19], [24]). Условие U3 эквивалентно ограниченности резольвенты оператора $H$ в точке $\lambda=m^{2}$ (см. [31; теорема 7.2]).

1.3. Результаты. Обозначим $\psi_{v}=s(\gamma x), \pi_{v}=-v \psi_{v}^{\prime}(x)$. Основным результатом для уравнения (1.1) является следующая солитонная асимптотика:

$(\psi(x, t), \dot{\psi}(x, t)) \sim\left(\psi_{v_{ \pm}}\left(x-v_{ \pm} t-q_{ \pm}\right), \pi_{v_{ \pm}}\left(x-v_{ \pm} t-q_{ \pm}\right)\right)+W_{0}(t) \Phi_{ \pm}, \quad t \rightarrow \pm \infty$,

для решений с начальными данными, близкими к некоторому кинку. Здесь $W_{0}(t)$ - динамическая группа свободного уравнения Клейна-Гордона, $\Phi_{ \pm}-$ асимптотические состояния рассеяния. Слагаемые $W_{0}(t) \Phi_{ \pm}$соответствуют дисперсионным волнам, уносящим энергию в бесконечность. Асимптотика (1.6) справедлива в глобальной энергетической норме пространства Соболева $H^{1}(\mathbb{R}) \oplus$ $L^{2}(\mathbb{R})$.

ЗАмечАниЕ 1.2. Асимптотику (1.6) можно интерпретировать как взаимодействие входящего солитона с траекторией $v_{-} t+q_{-}$с входящей дисперсионной волной $W_{0}(t) \Phi_{-}$, в результате которого рождаются исходящий солитон с новой 
траекторией $v_{+} t+q_{+}$и новая исходящая дисперсионная волна $W_{0}(t) \Phi_{+}$. Это взаимодействие определяет (нелинейный) оператор рассеяния $\mathbf{S}:\left(v_{-}, q_{-}, \Phi_{-}\right) \mapsto$ $\left(v_{+}, q_{+}, \Phi_{+}\right)$. Однако описание области определения этого оператора остается открытой проблемой, так же как и описание его области значений.

Мы докажем асимптотику (1.6) при двух различных вариантах условий на дискретный спектр.

D1. Дискретный спектр оператора $H$ состоит ровно из одной точки $\lambda_{0}=0$.

D2. Дискретный спектр оператора $H$ cостоит из двух точек: $\lambda_{0}=0 u$ $\lambda_{1} \in\left(0, m^{2}\right)$, причем

$$
4 \lambda_{1}>m^{2} .
$$

Во втором случае будем также предполагать условие невырожденности, или так называемое золотое правило Ферми, означающее эффективное взаимодействие нелинейного члена с непрерывным спектром. Это взаимодействие обеспечивает рассеяние энергии в бесконечность (см. условие (10.0.11) в [7] или условие (1.11) в [28]). Для уравнения (1.1) золотое правило Ферми имеет вид

F.

$$
\int \varphi_{4 \lambda_{1}}(x) F^{\prime \prime}(s(x)) \varphi_{\lambda_{1}}^{2}(x) d x \neq 0
$$

где $\varphi_{\lambda_{1}}$ - собственная функция, соответствующая собственному значению $\lambda_{1}$, а $\varphi_{4 \lambda_{1}}$ - нечетная собственная функция непрерывного спектра,

соответствующая точке $4 \lambda_{1} \in\left(m^{2}, \infty\right)$.

Первый случай рассматривается в главе I, а второй - в главе II. Для простоты изложения во втором случае мы рассмотрели только нечетные решения уравнения (1.1) и, соответственно, доказали асимптотическую устойчивость стоячего кинка, т. е. при $v=0$ и $q=0$. Асимптотическая устойчивость движущихся кинков при условии D2 может быть получена объединением методов обеих глав.

В третьей главе строятся примеры нелинейностей, удовлетворяющих нашим спектральным условиям. Отметим, что в большинстве работ, посвященных асимптотической устойчивости солитонов, также накладывается ряд условий на спектральные свойства соответствующей линеаризованной динамики. Однако почти везде эти свойства только постулируются, и примеры нелинейностей, для которых они справедливы, в большинстве случаев неизвестны. Мы показываем, как построить примеры потенциалов, для которых можно проверить все нужные спектральные условия: свойства дискретного спектра линеаризованного уравнения, отсутствие резонанса и золотое правило Ферми.

1.4. Методы. Для доказательства асимптотической устойчивости солитонов в работах [5]-[9], [16], [40] используется общая стратегия, характерная для большинства работ в данном направлении. Эта стратегия основана на методах симплектической геометрии для гамильтоновых систем в гильбертовом пространстве и спектральной теории несамосопряженных операторов. Применяются, в частности, симплектическая проекция на солитонное многообразие и симплектически ортогональные направления, разделение динамики вдоль солитонного многообразия и в трансверсальном направлении, убывание для 
трансверсальной линеаризованной динамики, модуляционные уравнения для солитонных параметров, нормальные формы Пуанкаре, метод мажорант и т. д. Отметим, что симплектическая проекция позволяет исключить неустойчивые направления, соответствующие нулевому дискретному спектру линеаризованной динамики. Эти методы представляют собой современное развитие теории устойчивости Ляпунова.

Подобная стратегия применима и для релятивистских уравнений. Однако асимптотическая устойчивость кинков для этих уравнений не была установлена в течение долгого времени. Одна из причин заключается в том, что не было получено достаточно быстрое убывание решений одномерного линейного уравнения Клейна-Гордона с потенциалом, а хорошо известное убывание $\sim t^{-1 / 2}$, справедливое для решений свободного уравнения, недостаточно для применяемых методов (см. дискуссию во введении работы [9]). Поэтому первым нашим результатом в этом направлении было доказательство быстрого убывания $\sim t^{-3 / 2}$ в весовых энергетических нормах для проекции решения на непрерывный спектр при условии отсутствия собственных значений и резонансов в концевых точках непрерывного спектра [19], [24], [26].

Кроме того, несмотря на общую схему, многие утверждения и их доказательства существенно отличаются в связи со спецификой релятивистских уравнений, а некоторые являются абсолютно новыми. В частности, новыми являются следующие оценки.

I. Оценки, характеризующие скорость роста моментов решений нелинейного уравнения Клейна-Гордона (см. [27; приложение А]), являющиеся релятивистской версией оценок [7; $(1.2 .5)]$, используемых в [7] для нелинейного уравнения Шрёдингера.

II. Релятивистская версия (4.27) оценок решений в $L^{1}-L^{\infty}$-нормах.

Убывание в весовых энергетических нормах и оценки I-II играют важную роль в получении соответствующих неравенств для мажорант. Они позволяют также получить убывание трансверсальной компоненты линеаризованного на солитоне уравнения, что гарантирует излучение энергии в бесконечность, обеспечивающее асимптотическую устойчивость солитонного многообразия.

Заметим, что в наших работах [27], [28] рассмотрен несколько более общий случай. А именно, вместо условия U2 в этих работах предполагается, что

$$
U(\psi)=\frac{m^{2}}{2}(\psi \mp a)^{2}+\mathscr{O}\left(|\psi \mp a|^{K}\right), \quad \psi \rightarrow \pm a,
$$

с некоторым $K>13$. При этом доказательство асимптотической устойчивости кинков отличается только небольшими техническими деталями.

1.5. Открытые проблемы. Легко проверить, что известный потенциал Гинзбурга-Ландау $U_{\mathrm{GL}}(\psi)=\left(\psi^{2}-a^{2}\right)^{2} /\left(4 a^{2}\right)$ удовлетворяет условию (1.9) с $m^{2}=2$ и $K=3$, а также условиям $\mathbf{D 2}$ и $\mathbf{F}$. Однако концевая точка спектра $\lambda=2$ является резонансом для соответствующего линеаризованного оператора. Этот факт является основной причиной того, что асимптотическая устойчивость кинков для уравнения с потенциалом $U_{\mathrm{GL}}$ до сих пор не доказана. 
Численное моделирование А. Винниченко с соавторами [22] демонстрирует асимптотики вида (1.6) и более общие:

$(\psi(x, t), \dot{\psi}(x, t)) \sim \sum_{k=1}^{N}\left(\psi_{v_{ \pm}^{k}}\left(x-v_{ \pm}^{k} t-q_{ \pm}^{k}\right), \pi_{v_{ \pm}^{k}}\left(x-v_{ \pm}^{k} t-q_{ \pm}^{k}\right)\right)+W_{0}(t) \Phi_{ \pm}, \quad t \rightarrow \pm \infty$,

для общих нелинейных гиперболических уравнений и любых начальных состояний конечной энергии. Однако доказательство таких асимптотик в настоящее время является открытой проблемой. Такие асимптотики тесно связаны с проблемой устойчивости элементарных частиц и корпускулярно-волновой двойственностью в контексте нелинейной теории Гейзенберга [13], [14].

1.6. О распределении материала. Работа организована следующим образом. В первой главе излагаются методы доказательства асимптотической устойчивости бегущих кинков в случае выполнения условия D1, т. е. при отсутствии дополнительного дискретного спектра. В разделе 2 даются необходимые определения и формулируется главный результат. Раздел 3 посвящен симплектической структуре солитонного многообразия, а раздел 4 - линеаризации решения на кинке и свойствам линеаризованного уравнения. В разделе 5 мы разделяем динамику на две компоненты: вдоль солитонного многообразия и в трансверсальном направлении. В разделе 6 изучаются модуляционные уравнения для параметров солитона. Схема доказательства долговременного убывания трансверсальной компоненты приводится в разделе 7. В разделе 8 мы получаем солитонную асимптотику (1.6).

Во второй главе рассмотрен случай, когда оператор $H$ имеет дополнительный дискретный спектр, удовлетворяющий условию D2. Мы рассматриваем только нечетные решения и доказываем асимптотическую устойчивость стоячего кинка соответствующего $v=q=0$. В разделе 10 приводятся свойства линеаризованного уравнения. В разделе 11 мы выводим динамические уравнения для дискретной и непрерывной компонент решения, а в разделе 12 находим нормальные формы Пуанкаре для этих уравнений. Раздел 13 посвящен мажорантам и их оценкам. В разделе 14 выводится солитонная асимптотика.

В третьей главе приводятся примеры нелинейных потенциалов, удовлетворяющих спектральным условиям первой и второй глав работы.

\section{Глава I. Бегущие солитоны}

В этой главе мы рассматриваем асимптотическую устойчивость движущихся солитонов в случае отсутствия ненулевого дискретного спектра линеаризованной динамики.

\section{2. Формулировка главного результата}

Запишем уравнение (1.1) в виде системы двух уравнений первого порядка

$$
\left\{\begin{array}{l}
\dot{\psi}(x, t)=\pi(x, t) \\
\dot{\pi}(x, t)=\psi^{\prime \prime}(x, t)+F(\psi(x, t)), \quad x \in \mathbb{R},
\end{array}\right.
$$


где $F(\psi)=-U^{\prime}(\psi)$ и все производные понимаются в смысле распределений. Эта система является гамильтоновой, и соответствующий гамильтониан имеет вид

$$
\mathscr{H}(\psi, \pi)=\int\left[\frac{|\pi(x)|^{2}}{2}+\frac{\left|\psi^{\prime}(x)\right|^{2}}{2}+U(\psi(x))\right] d x .
$$

Задачу Коши для системы (2.1), запишем в векторном виде:

$$
\dot{Y}(t)=\mathscr{F}(Y(t)), \quad t \in \mathbb{R}, \quad Y(0)=Y_{0},
$$

где $Y(t)=(\psi(t), \pi(t)), Y_{0}=\left(\psi_{0}, \pi_{0}\right)$. Солитонные решения также запишем в векторном виде:

$$
Y_{q, v}(t)=\left(\psi_{v}(x-v t-q), \pi_{v}(x-v t-q)\right), \quad q \in \mathbb{R}, \quad v \in(-1,1),
$$

где

$$
\psi_{v}(x)=s(\gamma x), \quad \pi_{v}(x)=-v \psi_{v}^{\prime}(x) .
$$

Определим солитонное состояние $S(\sigma):=\left(\psi_{v}(x-b), \pi_{v}(x-b)\right)$ с произвольными параметрами $\sigma:=(b, v)$, где $b \in \mathbb{R}$ и $v \in(-1,1)$. Очевидно, что солитон $(2.4)$ можно представить в виде $S(\sigma(t))$, где

$$
\sigma(t)=(b(t), v(t))=(v t+q, v) .
$$

Солитонное многообразие состоит из всех солитонных состояний:

$$
\mathscr{S}:=\{S(\sigma): \sigma \in \Sigma:=\mathbb{R} \times(-1,1)\} .
$$

Определим также фазовое пространство для задачи Коши (2.3). Для любых $\alpha \in \mathbb{R}, p \geqslant 1$ и $k=0,1,2, \ldots$ обозначим через $W_{\alpha}^{p, k}$ весовое соболевское пространство функций с конечными нормами

$$
\|\psi\|_{W_{\alpha}^{p, k}}=\sum_{i=0}^{k}\left\|(1+|x|)^{\alpha} \psi^{(i)}\right\|_{L^{p}} .
$$

Обозначим $H_{\alpha}^{k}:=W_{\alpha}^{2, k}, L_{\alpha}^{2}:=H_{\alpha}^{0}$ и введем пространства $E_{\alpha}:=H_{\alpha}^{1} \oplus L_{\alpha}^{2}$ и $W:=W_{0}^{1,2} \oplus W_{0}^{1,1}$ векторных функций $Y=(\psi, \pi)$ с конечными нормами

$$
\|Y\|_{E_{\alpha}}=\|\psi\|_{H_{\alpha}^{1}}+\|\pi\|_{L_{\alpha}^{2}} \quad \text { и } \quad\|Y\|_{W}=\|\psi\|_{W_{0}^{2,1}}+\|\pi\|_{W_{0}^{1,1}} .
$$

Мы будем работать в фазовом пространстве $\mathscr{E}:=E+\mathscr{S}$, где $E=E_{0}$, а $\mathscr{S}$ определено в (2.7). Метрика в $\mathscr{E}$ определяется следующим образом:

$$
\rho_{\mathscr{E}}\left(Y_{1}, Y_{2}\right)=\left\|Y_{1}-Y_{2}\right\|_{E}, \quad Y_{1}, Y_{2} \in \mathscr{E} .
$$

Очевидно, что гамильтониан (2.2) непрерывен на фазовом пространстве $\mathscr{E}$. Используя методы работ [29], [35], [41], легко доказать, что

(i) для любых начальных данных $Y_{0} \in \mathscr{E}$ существует единственное решение $Y(t) \in C(\mathbb{R}, \mathscr{E})$ задачи (2.3);

(ii) для любого $t \in \mathbb{R}$ отображение $U(t): Y_{0} \mapsto Y(t)$ непрерьвно в $\mathscr{E}$;

(iii) справедлив закон сохранения энергии:

$$
\mathscr{H}(Y(t))=\mathscr{H}\left(Y_{0}\right), \quad t \in \mathbb{R} .
$$

Главным результатом первой главы является следующая теорема. 
ТеОрема 2.1. Пусть выполнены условия U1-U3 и D1, и пусть $Y(t)-p e-$ шение задачи Коши (2.3) с начальными данными $Y_{0} \in \mathscr{E}$, близкими к некоторому кинку $S\left(\sigma_{0}\right)=S_{q_{0}, v_{0}}$ :

$$
Y_{0}=S\left(\sigma_{0}\right)+X_{0}, \quad d_{0}:=\left\|X_{0}\right\|_{E_{\beta} \cap W} \ll 1,
$$

где $\beta>5 / 2$. Тогда при достаточно мальх $d_{0}$ справедлива следующая асимптотика:

$Y(x, t)=\left(\psi_{v_{ \pm}}\left(x-v_{ \pm} t-q_{ \pm}\right), \pi_{v_{ \pm}}\left(x-v_{ \pm} t-q_{ \pm}\right)\right)+W_{0}(t) \Phi_{ \pm}+r_{ \pm}(x, t), \quad t \rightarrow \pm \infty$

с некоторыми постоянными $v_{ \pm} u q_{ \pm}$. Здесъ $W_{0}(t)$ - динамическая группа свободного уравнения Клейна-Гордона $и \Phi_{ \pm} \in E$ - асимптотические состояния рассеяния. Кроме того,

$$
\left\|r_{ \pm}(t)\right\|_{E}=\mathscr{O}\left(|t|^{-1 / 2}\right), \quad t \rightarrow \pm \infty
$$

Заметим, что достаточно проверить асимптотику (2.9) при $t \rightarrow+\infty$, так как система (2.1) обратима во времени.

\section{3. Симплектическая проекция}

3.1. Симплектическая структура и гамильтонова форма. Отметим, что система (2.3) является гамильтоновой системой, т. е. ее можно записать в виде

$$
\dot{Y}=J \mathscr{D} \mathscr{H}(Y), \quad J:=\left(\begin{array}{cc}
0 & 1 \\
-1 & 0
\end{array}\right),
$$

где $\mathscr{D} \mathscr{H}$ - производная Фреше гамильтониана (2.2). Отождествим касательное пространство к $\mathscr{E}$ в произвольной точке с пространством $E$ и рассмотрим симплектическую форму $\Omega$ на $E$ :

$$
\Omega\left(Y_{1}, Y_{2}\right)=\left\langle Y_{1}, J Y_{2}\right\rangle, \quad Y_{1}, Y_{2} \in E,
$$

где $\left\langle Y_{1}, Y_{2}\right\rangle:=\left\langle\psi_{1}, \psi_{2}\right\rangle+\left\langle\pi_{1}, \pi_{2}\right\rangle$ и $\left\langle\psi_{1}, \psi_{2}\right\rangle=\int \psi_{1}(x) \psi_{2}(x) d x$. Очевидно, что форма $\Omega$ невырождена, т. е. если $\Omega\left(Y_{1}, Y_{2}\right)=0$ для любого $Y_{2} \in E$, то $Y_{1}=0$.

Запись $Y_{1} \nmid Y_{2}$ означает, что векторы $Y_{1} \in E$ и $Y_{2} \in E$ симплектически ортогональны, т. е. $\Omega\left(Y_{1}, Y_{2}\right)=0$. Будем говорить, что оператор проектирования $\mathbf{P}: E \rightarrow E$ симплектически ортогонален, если $Y_{1} \nmid Y_{2}$ для $Y_{1} \in \operatorname{Ker} \mathbf{P}$ и $Y_{2} \in \operatorname{Range} \mathbf{P}$.

3.2. Симплектическая проекция на солитонное многообразие. Касательное пространство $\mathscr{T}_{S(\sigma)} \mathscr{S}$ к многообразию $\mathscr{S}$ в точке $S(\sigma)$ порождается векторами

$$
\begin{aligned}
& \tau_{1}=\tau_{1}(v):=\partial_{b} S(\sigma)=\left(-\psi_{v}^{\prime}(y),-\pi_{v}^{\prime}(y)\right), \\
& \tau_{2}=\tau_{2}(v):=\partial_{v} S(\sigma)=\left(\partial_{v} \psi_{v}(y), \partial_{v} \pi_{v}(y)\right),
\end{aligned}
$$


которые в "движущейся системе координат" $y:=x-b$ образуют базис в пространстве $\mathscr{T}_{S(\sigma)} \mathscr{S}$. Подчеркнем, что функции $\tau_{j}$ зависят от переменной $y$, а не от переменной $x$. Из формулы (2.5) следует, что для любого $\alpha \in \mathbb{R}$ и $v \in(-1,1)$

$$
\tau_{j}(v) \in E_{\alpha}, \quad j=1,2 .
$$

Покажем, что симплектическая форма $\Omega$ невырождена на касательном пространстве $\mathscr{T}_{S(\sigma)} \mathscr{S}$. Для этого найдем явные выражения для векторов $\tau_{1}$ и $\tau_{2}$. Из определения (2.5) функций $\psi_{v}(y)$ и $\pi_{v}(y)$ следует, что

$$
\tau_{1}=\left(-\gamma s^{\prime}(\gamma y), v \gamma^{2} s^{\prime \prime}(\gamma y)\right), \quad \tau_{2}=\left(v y \gamma^{3} s^{\prime}(\gamma y),-\gamma^{3} s^{\prime}(\gamma y)-v^{2} y \gamma^{4} s^{\prime \prime}(\gamma y)\right) .
$$

Следовательно,

$$
\Omega\left(\tau_{1}, \tau_{2}\right)=\left\langle\tau_{1}^{1}, \tau_{2}^{2}\right\rangle-\left\langle\tau_{1}^{2}, \tau_{2}^{1}\right\rangle=\gamma^{4}\left\langle s^{\prime}(\gamma y), s^{\prime}(\gamma y)\right\rangle>0 .
$$

Это означает, что $\mathscr{T}_{S(\sigma)} \mathscr{S}$ является симплектическим подпространством. В малой окрестности солитонного многообразия $\mathscr{S}$ определена "симплектическая ортогональная проекция" на $\mathscr{S}$. Доказательство этого факта довольно простое и подробно изложено в работах [16], [27], [28]. Дадим точную формулировку.

Лемма 3.1. Для любого $\alpha \in \mathbb{R}$ справедливы следующие утвержсдния.

(i) Существуют окрестность $\mathscr{O}_{\alpha}(\mathscr{S})$ многообразия $\mathscr{S}$ в пространстве $E_{\alpha} u$ отображение $\Pi: \mathscr{O}_{\alpha}(\mathscr{S}) \rightarrow \mathscr{S}$ такие, что $\Pi$ равномерно непрерывно на $\mathscr{O}_{\alpha}(\mathscr{S})$ в метрике $E_{\alpha}$. Кроме того,

$$
\Pi Y=Y \quad \text { для } Y \in \mathscr{S}, \quad Y-S \nmid \mathscr{T}_{S} \mathscr{S}, \quad \text { где } S=\Pi Y .
$$

(ii) Для всех $q \in \mathbb{R}$ окрестность $\mathscr{O}_{\alpha}(\mathscr{S})$ инвариантна относительно сдвигов

$$
T_{q}:(\psi(x), \pi(x)) \mapsto(\psi(x+q), \pi(x+q))
$$

u, кроме того, если $Y \in \mathscr{O}_{\alpha}(\mathscr{S})$, то $\Pi T_{q} Y=T_{q} \Pi$.

(iii) Для любого $\bar{v}<1$ существует $r_{\alpha}(\bar{v})>0$ такое, что если $|v| \leqslant \bar{v} u$ $\|X\|_{E_{\alpha}}<r_{\alpha}(\bar{v})$, mо $S(\sigma)+X \in \mathscr{O}_{\alpha}(\mathscr{S})$ nри всех $b \in \mathbb{R}$.

Назовем отображение $\Pi$ симплектическим ортогональным проектором на $\mathscr{S}$.

\section{4. Линеаризация на солитонном многообразии}

Будем искать решение системы (2.1) в виде суммы

$$
Y(t)=S(\sigma(t))+X(t) .
$$

Здесь $S(\sigma(t))$ - солитон с параметрами $\sigma(t)=(b(t), v(t))$, где $b(t) \in \mathbb{R}$ и $v(t) \in$ $(-1,1)$ - некоторые гладкие функции от переменной $t \in \mathbb{R}$. Для компонент векторных функций $Y=(\psi, \pi)$ и $X=(\Psi, \Pi)$ уравнение (4.1) выглядит следующим образом:

$$
\left\{\begin{array}{l}
\psi(x, t)=\psi_{v(t)}(x-b(t))+\Psi(x-b(t), t), \\
\pi(x, t)=\pi_{v(t)}(x-b(t))+\Pi(x-b(t), t) .
\end{array}\right.
$$


Подставив эти уравнения в систему (2.1), получим в “движущейся системе координат" $y=x-b(t)$ следующие уравнения:

$$
\begin{aligned}
\dot{\psi} & =\dot{v} \partial_{v} \psi_{v}(y)-\dot{b} \psi_{v}^{\prime}(y)+\dot{\Psi}(y, t)-\dot{b} \Psi^{\prime}(y, t)=\pi_{v}(y)+\Pi(y, t), \\
\dot{\pi} & =\dot{v} \partial_{v} \pi_{v}(y)-\dot{b} \pi_{v}^{\prime}(y)+\dot{\Pi}(y, t)-\dot{b} \Pi^{\prime}(y, t) \\
& =\psi_{v}^{\prime \prime}(y)+\Psi^{\prime \prime}(y, t)+F\left(\psi_{v}(y)+\Psi(y, t)\right)
\end{aligned}
$$

Так как уравнения для солитона в переменной $y=x-b(t)$ имеют вид

$$
-v \psi_{v}^{\prime}(y)=\pi_{v}(y), \quad-v \pi_{v}^{\prime}(y)=\psi_{v}^{\prime \prime}(y)+F\left(\psi_{v}(y)\right),
$$

то из (4.3) немедленно следуют уравнения для функций $\Psi(t)$ и $\Pi(t)$ :

$$
\begin{gathered}
\dot{\Psi}(y, t)=\Pi(y, t)+\dot{b} \Psi^{\prime}(y, t)+(\dot{b}-v) \psi_{v}^{\prime}(y)-\dot{v} \partial_{v} \psi_{v}(y), \\
\dot{\Pi}(y, t)=\Psi^{\prime \prime}(y, t)+\dot{b} \Pi^{\prime}(y, t)+(\dot{b}-v) \pi_{v}^{\prime}(y)-\dot{v} \partial_{v} \pi_{v}(y) \\
+F\left(\psi_{v}(y)+\Psi(y, t)\right)-F\left(\psi_{v}(y)\right) .
\end{gathered}
$$

Запишем уравнения (4.5) в виде

$$
\dot{X}(t)=A(t) X(t)+T(t)+\mathscr{N}(t), \quad t \in \mathbb{R},
$$

где слагаемое $T(t)$ не зависит от $X$, а слагаемое $\mathscr{N}(t)$ по крайней мере квадратично относительно $X$. Линейный оператор $A(t)=A_{v, w}(t)$ зависит от двух параметров, $v=v(t)$ и $w=\dot{b}(t)$, и может быть записан в виде

$$
A_{v, w}\left(\begin{array}{c}
\Psi \\
\Pi
\end{array}\right):=\left(\begin{array}{cc}
w \nabla & 1 \\
\Delta+F^{\prime}\left(\psi_{v}\right) & w \nabla
\end{array}\right)\left(\begin{array}{c}
\Psi \\
\Pi
\end{array}\right)=\left(\begin{array}{cc}
w \nabla & 1 \\
\Delta-m^{2}-V_{v}(y) & w \nabla
\end{array}\right)\left(\begin{array}{c}
\Psi \\
\Pi
\end{array}\right),
$$

где $\nabla=d / d x, \Delta=d^{2} / d x^{2}$ и потенциал $V_{v}(y)$ определяется равенством

$$
V_{v}(y)=-F^{\prime}\left(\psi_{v}\right)-m^{2} .
$$

Далее выпишем выражения для $T(t)$ и $\mathscr{N}(t)=\mathscr{N}(\sigma, X)$ :

$$
T=\left(\begin{array}{c}
(w-v) \psi_{v}^{\prime}-\dot{v} \partial_{v} \psi_{v} \\
(w-v) \pi_{v}^{\prime}-\dot{v} \partial_{v} \pi_{v}
\end{array}\right), \quad \mathscr{N}(\sigma, X)=\left(\begin{array}{c}
0 \\
N(v, \Psi)
\end{array}\right)
$$

где $v=v(t), w=w(t), \sigma=\sigma(t)=(b(t), v(t)), X=X(t)=(\Psi(t), \Pi(t))$ и

$$
N(v, \Psi)=F\left(\psi_{v}+\Psi\right)-F\left(\psi_{v}\right)-F^{\prime}\left(\psi_{v}\right) \Psi
$$

Заметим, что слагаемое $A(t) X(t)$ в правой части уравнения (4.6) линейно относительно $X(t)$, а $\mathscr{N}(t)$ является членом высшего порядка относительно $X(t)$. С другой стороны, слагаемое $T(t)$ имеет нулевой порядок и не обращается в нуль при $X(t)=0$, так как $S(\sigma(t))$ не является кинком, если не выполнено условие (2.6). Заметим также, что из формул (3.3) и (4.9) следует, что

$$
T(t)=-(w-v) \tau_{1}-\dot{v} \tau_{2} .
$$

Поэтому $T(t) \in \mathscr{T}_{S(\sigma(t))} \mathscr{S}$ при всех $t \in \mathbb{R}$. Это означает, что нелинейная динамика вдоль солитонного многообразия имеет неустойчивый характер. 
4.1. Гамильтонова структура и спектр. Изучим спектральные свойства оператора $A_{v, w}$. Рассмотрим подробнее линейное уравнение

$$
\dot{X}(t)=A_{v, w} X(t), \quad t \in \mathbb{R},
$$

с некоторыми фиксированными $v \in(-1,1)$ и $w \in \mathbb{R}$. Определим пространство $E^{+}:=H^{2}(\mathbb{R}) \oplus H^{1}(\mathbb{R})$. Гамильтоновы свойства уравнения (4.12) описываются следующей леммой.

Лемма 4.1. (i) Для любых $v \in(-1,1) u w \in \mathbb{R}$ уравнение (4.12) можно записать в гамильтоновой форме

$$
\dot{X}(t)=J \mathscr{D} \mathscr{H}_{v, w}(X(t)), \quad t \in \mathbb{R},
$$

где $\mathscr{D} \mathscr{H}_{v, w}$ - производная Фреше гамильтониана

$$
\mathscr{H}_{v, w}(X)=\frac{1}{2} \int\left[|\Pi|^{2}+\left|\Psi^{\prime}\right|^{2}+\left(m^{2}+V_{v}\right)|\Psi|^{2}\right] d y+\int \Pi w \Psi^{\prime} d y .
$$

(ii) Для решениц $X(t) \in C^{1}\left(\mathbb{R}, E^{+}\right)$справедлив закон сохранения энергии:

$$
\mathscr{H}_{v, w}(X(t))=\text { const }, \quad t \in \mathbb{R} .
$$

(iii) Выполняется соотношение кососимметричности:

$$
\Omega\left(A_{v, w} X_{1}, X_{2}\right)=-\Omega\left(X_{1}, A_{v, w} X_{2}\right), \quad X_{1}, X_{2} \in E .
$$

Доказательство этой леммы можно найти в [27].

Рассмотрим действие оператора $A_{v, w}$ на касательные векторы $\tau=\tau_{j}(v)$ к солитонному многообразию. Дифференцируя уравнения (4.4) по $b$ и $v$, получим

$$
\begin{aligned}
-v \psi_{v}^{\prime \prime} & =\pi_{v}^{\prime}, & -v \pi_{v}^{\prime \prime} & =\psi_{v}^{\prime \prime \prime}+F^{\prime}\left(\psi_{v}\right) \psi_{v}^{\prime}, \\
-\psi_{v}^{\prime}-v \partial_{v} \psi_{v}^{\prime} & =\partial_{v} \pi_{v}, & -\pi_{v}^{\prime}-v \partial_{v} \pi_{v}^{\prime} & =\partial_{v} \psi_{v}^{\prime \prime}+F^{\prime}\left(\psi_{v}\right) \partial_{v} \psi_{v} .
\end{aligned}
$$

Поэтому

$$
A_{v, w}\left(\begin{array}{c}
-\psi_{v}^{\prime} \\
-\pi_{v}^{\prime}
\end{array}\right)=\left(\begin{array}{c}
(v-w) \psi_{v}^{\prime \prime} \\
(v-w) \pi_{v}^{\prime \prime}
\end{array}\right), \quad A_{v, w}\left(\begin{array}{c}
\partial_{v} \psi_{v} \\
\partial_{v} \pi_{v}
\end{array}\right)=\left(\begin{array}{c}
(w-v) \partial_{v} \psi_{v}^{\prime} \\
(w-v) \partial_{v} \pi_{v}^{\prime}
\end{array}\right)+\left(\begin{array}{c}
-\psi_{v}^{\prime} \\
-\pi_{v}^{\prime}
\end{array}\right) .
$$

В результате получим, что

$$
A_{v, w}\left[\tau_{1}\right]=(w-v) \tau_{1}^{\prime}, \quad A_{v, w}\left[\tau_{2}\right]=(w-v) \tau_{2}^{\prime}+\tau_{1} .
$$

Рассмотрим теперь оператор $A_{v}=A_{v, v}$, соответствующий $w=v$ :

$$
A_{v}:=\left(\begin{array}{cc}
v \nabla & 1 \\
\Delta-m^{2}-V_{v}(y) & v \nabla
\end{array}\right),
$$

и изучим его спектральные свойства. Непрерывный спектр оператора $A_{v}$ совпадает с интервалом $\Gamma:=(-i \infty,-i m / \gamma] \cup[i m / \gamma, i \infty)$. Из равенств (4.14) следует, 
что касательный вектор $\tau_{1}(v)$ является собственным вектором оператора $A_{v}$, соответствующим нулевому собственному значению, а касательный вектор $\tau_{2}(v)$ является присоединенным вектором, т. е.

$$
A_{v}\left[\tau_{1}(v)\right]=0, \quad A_{v}\left[\tau_{2}(v)\right]=\tau_{1}(v)
$$

Проверим, что корневое пространство оператора $A_{v}$, соответствующее нулевому собственному значению, является двумерным пространством. Для этого достаточно проверить, что уравнение $A_{v}[u]=\tau_{2}$ не имеет ненулевых решений из пространства $L^{2} \oplus L^{2}$. Рассмотрим это уравнение подробнее:

$$
\left(\begin{array}{cc}
v \nabla & 1 \\
\Delta-m^{2}-V_{v}(y) & v \nabla
\end{array}\right)\left(\begin{array}{l}
u_{1} \\
u_{2}
\end{array}\right)=\left(\begin{array}{c}
v \gamma^{3} y s^{\prime}(\gamma y) \\
-\gamma^{3} s^{\prime}(\gamma y)-v^{2} \gamma^{4} y s^{\prime \prime}(\gamma y)
\end{array}\right)
$$

Из первого уравнения найдем $u_{2}=v \gamma^{3} y s^{\prime}(\gamma y)-v \nabla u_{1}$. Подставив это выражение во второе уравнение, получим

$$
H_{v} u_{1}=-\gamma^{3}\left(1+v^{2}\right) s^{\prime}(\gamma y)-2 v^{2} \gamma^{4} y s^{\prime \prime}(\gamma y),
$$

где $H_{v}=-\left(1 / \gamma^{2}\right) d^{2} / d y^{2}+m^{2}+V_{v}(y)-$ модифицированный оператор Шрёдингера. Положив $u_{1}=-(1 / 2) v^{2} \gamma^{5} y^{2} s^{\prime}(\gamma y)+\widetilde{u}_{1}$, преобразуем последнее уравнение к виду

$$
H_{v} \widetilde{u}_{1}=-\gamma^{2} \psi_{v}^{\prime}
$$

ЗАмечАниЕ 4.2. Спектральные свойства операторов $H_{v}$ идентичны при всех $v \in(-1,1)$, так как из соотношения $V_{v}(x)=V_{0}(\gamma x)$ вытекает, что

$$
H_{v}=\mathscr{I}_{v}^{-1} H_{0} \mathscr{I}_{v}, \quad \text { где } \quad \mathscr{I}_{v}: \psi(x) \mapsto \psi\left(\frac{x}{\gamma}\right) .
$$

Это подобие операторов связано с релятивистской инвариантностью исходного уравнения (1.1). В частности, для оператора $H_{v}$ выполнены свойства Н1-H3 (с собственной функцией $\psi_{v}^{\prime}$ вместо $s^{\prime}$ ), а также свойства U3 и D1.

Так как точка $\lambda_{0}=0$ является точкой дискретного спектра оператора $H_{v}$ с собственной функцией $\psi_{v}^{\prime}$, то уравнение (4.18) означает, что функция $\widetilde{u}_{1}$ является присоединенной функцией оператора $H_{v}$. Однако это невозможно в силу самосопряженности этого оператора. Итак, мы доказали, что корневое пространство оператора $A_{v}$, соответствующее нулевому собственному значению, является двумерной жордановой клеткой.

Покажем, что оператор $A_{v}$ не имеет других собственных значений, кроме $\lambda=0$. Для этого рассмотрим спектральное уравнение

$$
\left(\begin{array}{cc}
v \nabla & 1 \\
\Delta-m^{2}-V_{v}(y) & v \nabla
\end{array}\right)\left(\begin{array}{l}
u_{1} \\
u_{2}
\end{array}\right)=\lambda\left(\begin{array}{l}
u_{1} \\
u_{2}
\end{array}\right) .
$$

Из первого уравнения найдем $u_{2}=-(v \nabla-\lambda) u_{1}$ и подставим во второе уравнение. Получим

$$
\left(H_{v}+\lambda^{2}-2 v \lambda \nabla\right) u_{1}=0
$$


Так как в силу условия D1 оператор $H_{0}=H$, соответствующий $v=0$, имеет только нулевое собственное значение, то и оператор $A_{0}$ тоже имеет только нулевое собственное значение. В случае $v \neq 0$ умножим скалярно обе части уравнения (4.20) на $u_{1}$ :

$$
\left\langle H_{v} u_{1}, u_{1}\right\rangle+\lambda^{2}\left\langle u_{1}, u_{1}\right\rangle=0 .
$$

Так как оператор $H_{v}$ самосопряженный, то $\lambda^{2}$ может быть только вещественным числом. Ненулевое собственное значение оператора $A_{v}$ может появиться при непрерывном изменении параметра $v$ как бифуркация либо из точки $\lambda=0$, либо из концевых точек $\pm i m / \gamma$ непрерывного спектра. Рассмотрим эти случаи отдельно.

(i) Бифуркация из точки $\lambda=0$ невозможна, так как это изолированная точка дискретного спектра и мы уже знаем, что соответствующее ей корневое подпространство является двумерным при всех значениях $v \in(-1,1)$.

(ii) Бифуркация из концевых точек также невозможна. В самом деле, порожденные концевыми точками собственные значения $\lambda$ могут быть только чисто мнимыми, так как $\lambda^{2}$ - вещественное число. Пусть $\lambda \in(-i m / \gamma, i m / \gamma)$ является собственным значением оператора $A_{v}$. Тогда $A_{v}[u]=\lambda u$, где $u=\left(u_{1}, u_{2}\right) \in$ $L^{2} \oplus L^{2}$ - соответствующая собственная функция. Введем новую функцию $p(x)=e^{\gamma^{2} v \lambda x} u(x)$. Очевидно, что $p=\left(p_{1}, p_{2}\right)$ также принадлежит пространству $L^{2} \oplus L^{2}$. Уравнение (4.20) для $p_{1}$ перепишется в виде $\left(H_{v}+\gamma^{2} \lambda^{2}\right) p_{1}=0$, где $-\gamma^{2} \lambda^{2} \in\left(0, m^{2}\right)$. В силу условия D1 это уравнение не имеет ненулевых решений из $L^{2}$.

Таким образом, мы установили, что оператор $A_{v}$ имеет только одно собственное значение $\lambda=0$.

4.2. Убывание трансверсальной линеаризованной динамики. Рассмотрим линеаризованное уравнение

$$
\dot{X}(t)=A_{v} X(t), \quad t \in \mathbb{R} .
$$

Обозначим через $\mathbf{P}_{v}^{\mathrm{d}}$ симплектический ортогональный проектор на касательное пространство $\mathscr{T}_{S(\sigma)} \mathscr{S}$, действующий в пространстве $E$. В силу линейности,

$$
\mathbf{P}_{v}^{\mathrm{d}} X=\sum p_{j l}(v) \tau_{j}(v) \Omega\left(\tau_{l}(v), X\right), \quad X \in E,
$$

где $p_{j l}(v)$ - некоторые гладкие коэффициенты. Заметим, что проектор $\mathbf{P}_{v}^{\mathrm{d}}$ в переменных $y=x-b$ не зависит от $b$. Положим $\mathbf{P}_{v}^{\mathrm{c}}:=\mathbf{I}-\mathbf{P}_{v}^{\mathrm{d}}$. Один из ключевых этапов доказательства асимптотической устойчивости солитонов состоит в установлении долговременного убывания решений трансверсального линеаризованного уравнения. Следующее предложение в случае $v=0$ доказано в работах [19], [24], а в случае $v \neq 0$ - в работе [26].

ПрЕДЛОЖЕНИЕ 4.3. Пусть выполнены условия U1-U3, и пусть $\beta>5 / 2$. Тогда для любого $X \in E_{\beta}$ справедливо следующее долговременное убывание в весовых нормах:

$$
\left\|e^{A_{v} t} \mathbf{P}_{v}^{\mathrm{c}} X\right\|_{E_{-\beta}} \leqslant C(v)(1+|t|)^{-3 / 2}\|X\|_{E_{\beta}}, \quad t \in \mathbb{R} .
$$


Здесь и всюду далее мы обозначаем через $e^{A_{v} t}$ динамическую группу уравнения (4.21). Из убывания (4.23) легко выводится равномерное по $x$ убывание, т. е. убывание в норме пространства $L^{\infty}$, справедливое для всех $X \in E_{\beta} \cap W$. Чтобы получить это убывание, применим проектор $\mathbf{P}_{v}^{\mathrm{c}}$ к обеим частям уравнения (4.21):

$$
\mathbf{P}_{v}^{\mathrm{c}} \dot{X}=A_{v} \mathbf{P}_{v}^{\mathrm{c}} X=A_{v}^{0} \mathbf{P}_{v}^{\mathrm{c}} X+\mathscr{V}_{v} \mathbf{P}_{v}^{\mathrm{c}} X
$$

где

$$
A_{v}^{0}=\left(\begin{array}{cc}
v \nabla & 1 \\
\Delta-m^{2} & v \nabla
\end{array}\right), \quad \mathscr{V}_{v}=\left(\begin{array}{cc}
0 & 0 \\
-V_{v} & 0
\end{array}\right) .
$$

Обозначим $Y=\mathbf{P}_{v}^{c} X$ и напишем представление Дюамеля для решения уравнения (4.24):

$$
e^{A_{v} t} Y=e^{A_{v}^{0} t} Y+\int_{0}^{t} e^{A_{v}^{0}(t-\tau)} \mathscr{V}_{v} e^{A_{v} \tau} Y d \tau, \quad t \in \mathbb{R} .
$$

Заметим, что $e^{A_{v}^{0} t} Z=e^{A_{0}^{0} t} T_{v t} Z$, где оператор сдвига $T_{v t}$ определен в (3.7). Следовательно,

$$
e^{A_{v} t} Y=e^{A_{0}^{0} t} T_{v t} Y+\int_{0}^{t} e^{A_{0}^{0}(t-\tau)} T_{v t}\left[\mathscr{V}_{v} e^{A_{v} \tau} Y\right] d \tau, \quad t \in \mathbb{R} .
$$

Так как потенциал $V_{v}$ имеет компактный носитель, то, применяя неравенство (265) из [36], неравенство Гёльдера, а также неравенство (4.23), получим следующую оценку для первой компоненты векторной функции $e^{A_{v} t} Y$ :

$$
\begin{aligned}
& \left\|\left(e^{A_{v} t} Y\right)_{1}\right\|_{L^{\infty}} \leqslant C(1+|t|)^{-1 / 2}\|Y\|_{W}+C \int_{0}^{t}(1+|t-\tau|)^{-1 / 2}\left\|\mathscr{V}_{v}\left(e^{A_{v} \tau} Y\right)_{1}\right\|_{W_{0}^{1,1}} d \tau \\
& \leqslant C(1+|t|)^{-1 / 2}\|X\|_{W}+C \int_{0}^{t}(1+|t-\tau|)^{-1 / 2}\left\|e^{A_{v} \tau} \mathbf{P}_{v}^{\mathrm{c}} X\right\|_{E_{-\beta}} d \tau \\
& \leqslant C(1+|t|)^{-1 / 2}\|X\|_{W}+C \int_{0}^{t}(1+|t-\tau|)^{-1 / 2}(1+|\tau|)^{-3 / 2}\|X\|_{E_{\beta}} d \tau \\
& \leqslant C(1+|t|)^{-1 / 2}\left(\|X\|_{W}+\|X\|_{E_{\beta}}\right) .
\end{aligned}
$$

Таким образом, для любого $\beta>5 / 2$ и $X \in E_{\beta} \cap W$ мы доказали, что

$$
\left\|\left(e^{A_{v} t} \mathbf{P}_{v}^{\mathrm{c}} X\right)_{1}\right\|_{L^{\infty}} \leqslant C(v)(1+|t|)^{-1 / 2}\left(\|X\|_{W}+\|X\|_{E_{\beta}}\right), \quad t \in \mathbb{R} .
$$

4.3. Оценки нелинейного члена. Выведем оценки для нелинейного члена $N(v, \Psi)$, определенного формулой (4.10). Обозначим через $\mathscr{R}(a)$ положительную функцию, ограниченную при достаточно малых значениях $a$. Применяя формулу Коши для остаточного члена, получим

$$
N=\frac{\Psi^{2}}{2} \int_{0}^{1}(1-\rho) F^{\prime \prime}\left(\psi_{v}+\rho \Psi\right) d \rho .
$$

Согласно условию U2, функция $F^{\prime \prime}(\psi)$ равна нулю в некоторой окрестности точек $\pm a$. Поэтому из неравенства Коши-Буняковского следует, что

$$
\|N\|_{L^{1}}=\mathscr{R}\left(\|\Psi\|_{L^{\infty}}\right)\|\Psi\|_{L^{\infty}}\|\Psi\|_{L_{-\beta}^{2}}=\mathscr{R}\left(\|\Psi\|_{L^{\infty}}\right)\|\Psi\|_{L^{\infty}}\|X\|_{E_{-\beta}} .
$$


Дифференцируя (4.28) по переменой $y$, получим

$$
N^{\prime}=\frac{\Psi^{2}}{2} \int_{0}^{1}(1-\rho)\left(\psi_{v}^{\prime}+\rho \Psi^{\prime}\right) F^{\prime \prime \prime}\left(\psi_{v}+\rho \Psi\right) d \rho+\Psi \Psi^{\prime} \int_{0}^{1}(1-\rho) F^{\prime \prime}\left(\psi_{v}+\rho \Psi\right) d \rho .
$$

Следовательно,

$$
\begin{aligned}
\left\|N^{\prime}\right\|_{L^{1}} & =\mathscr{R}\left(\|\Psi\|_{L^{\infty}}\right)\left[\|\Psi\|_{L^{\infty}}\|\Psi\|_{L_{-\beta}^{2}}+\|\Psi\|_{L^{\infty}}\left\|\Psi^{\prime}\right\|_{L_{-\beta}^{2}}\right] \\
& =\mathscr{R}\left(\|\Psi\|_{L^{\infty}}\right)\|\Psi\|_{L^{\infty}}\|X\|_{E_{-\beta}} .
\end{aligned}
$$

Таким образом, нелинейный член $N(v, \Psi)$ в норме пространства $W_{0}^{1,1}$ оценивается как

$$
\|N\|_{W_{0}^{1,1}}=\mathscr{R}\left(\|\Psi\|_{L^{\infty}}\right)\|\Psi\|_{L^{\infty}}\|X\|_{E_{-\beta}} .
$$

Кроме того, из формулы (4.28) следует аналогичная оценка для $N(v, \Psi)$ в норме пространства $L_{\beta}^{2}$. В результате получим следующую оценку:

$$
\|N\|_{L_{\beta}^{2} \cap W_{0}^{1,1}}=\mathscr{R}\left(\|\Psi\|_{L^{\infty}}\right)\|\Psi\|_{L^{\infty}}\|X\|_{E_{-\beta}} .
$$

\section{5. Симплектическое разбиение динамики}

Уравнение (4.6) получено без каких-либо дополнительных предположениях о параметрах $\sigma(t)=(v(t), b(t))$ в представлении (4.1). Теперь мы предположим, что $S(\sigma(t)):=\Pi Y(t)$. Это можно сделать, если при всех $t \geqslant 0$

$$
Y(t) \in \mathscr{O}_{\alpha}(\mathscr{S})
$$

где $\mathscr{O}_{\alpha}(\mathscr{S})$ - окрестность, определенная в лемме 3.1. Условие (5.1) выполнено для $t=0$ в силу предположения (2.8). Поэтому определены величины $S(\sigma(0))=$ $\Pi Y(0)$ и $X(0)=Y(0)-S(\sigma(0))$. Ниже мы покажем, что условие $(5.1)$ с $\alpha=-\beta$ выполнено при всех $t \geqslant 0$, если $d_{0}$ в $(2.8)$ достаточно мало. Выберем любое $\bar{v}<1$ такое, что

$$
|v(0)|<\bar{v}
$$

и обозначим через $r_{-\beta}(\bar{v})$ положительное число, определенное в лемме 3.1 , (iii), соответствующее $\alpha=-\beta$. Тогда $S(\sigma)+X \in \mathscr{O}_{-\beta}(\mathscr{S})$ при условии, что $|v|<\bar{v}$ и $\|X\|_{E_{-\beta}}<r_{-\beta}(\bar{v})$. Поэтому $S(\sigma(t))=\Pi Y(t)$ и функция $X(t)=Y(t)-S(\sigma(t))$ определена для таких $t \geqslant 0$, при которых

$$
|v(t)|<\bar{v} \quad \text { и } \quad\|X(t)\|_{E_{-\beta}}<r_{-\beta}(\bar{v}) .
$$

Выполнение условия (5.3) для всех $t \geqslant 0$ обосновывается при помощи стандартного понятия "момента выхода". Определим так называемые мажоранты

$$
m_{1}(t):=\sup _{s \in[0, t]}(1+s)^{3 / 2}\|X(s)\|_{E_{-\beta}}, \quad m_{2}(t):=\sup _{s \in[0, t]}(1+s)^{1 / 2}\|\Psi(s)\|_{L^{\infty}} .
$$

Здесь $X=(\Psi, \Pi)$. Обозначим $\nu=\bar{v}-|v(0)|$ и выберем некоторое фиксированное число $\varepsilon \in\left(0, r_{-\beta}(\bar{v})\right)$, значение которого мы уточним позднее. 
ОПРЕДЕлЕНиЕ 5.1. Момент выхода $t_{*}$ определяется следующим образом:

$$
t_{*}=\sup \left\{t:|v(t)-v(0)|<\nu, m_{j}(t)<\varepsilon, j=1,2\right\} .
$$

Заметим, что $m_{j}(0)<\varepsilon$, если $d_{0} \ll 1$. Наша задача - доказать, что $t_{*}=\infty$, если $d_{0}$ достаточно мало. Для этого достаточно установить, что при малых $d_{0}$ выполнены неравенства

$$
|v(t)-v(0)|<\frac{\nu}{2}, \quad m_{j}(t)<\frac{\varepsilon}{2}, \quad 0 \leqslant t<t_{*} .
$$

\section{6. Модуляционные уравнения}

В этом разделе мы выведем уравнения для солитонных параметров $\sigma(t)=$ $(b(t), v(t))$ симплектической проекции $S(\sigma(t))$ решения $Y(t)$ уравнения $(2.3)$. А именно, будем искать решение уравнения $(2.3)$ в виде $Y(t)=S(\sigma(t))+X(t)$, где $S(\sigma(t))=\Pi Y(t)$. Иначе говоря, мы будем предполагать выполнение условия симплектической ортогональности:

$$
X(t) \nmid \mathscr{T}_{S(\sigma(t))} \mathscr{S}, \quad t<t_{*} .
$$

Проекция П $Y(t)$ определена для $t<t_{*}$ по лемме 3.1, (iii). Перепишем условие (6.1) в виде

$$
\Omega\left(X(t), \tau_{j}(t)\right)=0, \quad j=1,2,
$$

где векторы $\tau_{j}(t)=\tau_{j}(\sigma(t))$, определенные в (3.3), порождают касательное пространство $\mathscr{T}_{S(\sigma(t))} \mathscr{S}$ к многообразию $\mathscr{S}$ в точке $S(\sigma(t))$. Для удобства мы вместо параметров $(b, v)$ будем использовать параметры $(c, v)$, где

$$
c(t)=b(t)-\int_{0}^{t} v(\tau) d \tau, \quad \dot{c}(t)=\dot{b}(t)-v(t)=w(t)-v(t) .
$$

Покажем, как получить модуляционные уравнения для параметров $c(t)$ и $v(t)$ из условий ортогональности (6.2). Для этого продифференцируем эти условия по переменной $t$ :

$$
0=\Omega\left(\dot{X}, \tau_{j}\right)+\Omega\left(X, \dot{\tau}_{j}\right)=\Omega\left(A_{v, w} X+T+\mathscr{N}, \tau_{j}\right)+\Omega\left(X, \dot{\tau}_{j}\right), \quad j=1,2 .
$$

Из соотношения кососимметричности (4.13) и равенств (4.14) следует, что

$$
\begin{aligned}
\Omega\left(A_{v, w} X, \tau_{1}\right) & =-\Omega\left(X, A_{v, w}\left[\tau_{1}\right]\right)=-\dot{c} \Omega\left(X, \tau_{1}^{\prime}\right), \\
\Omega\left(A_{v, w} X, \tau_{2}\right) & =-\Omega\left(X, A_{v, w}\left[\tau_{2}\right]\right)=-\Omega\left(X, \dot{c} \tau_{2}^{\prime}-\tau_{1}\right) \\
& =-\dot{c} \Omega\left(X, \tau_{2}^{\prime}\right)-\Omega\left(X, \tau_{1}\right)=-\dot{c} \Omega\left(X, \tau_{2}^{\prime}\right),
\end{aligned}
$$

так как $\Omega\left(X, \tau_{1}\right)=0$. Далее, из равенства (4.11) следует, что

$$
\Omega\left(T, \tau_{1}\right)=-\dot{v} \Omega\left(\tau_{2}, \tau_{1}\right)=\dot{v} \Omega\left(\tau_{1}, \tau_{2}\right), \quad \Omega\left(T, \tau_{2}\right)=-\dot{c} \Omega\left(\tau_{1}, \tau_{2}\right) .
$$

Используя равенства (6.5)-(6.7), перепишем уравнения (6.4) в виде

$$
\left\{\begin{array}{l}
0=-\dot{c} \Omega\left(X, \tau_{1}^{\prime}\right)+\dot{v}\left(\Omega\left(\tau_{1}, \tau_{2}\right)+\Omega\left(X, \partial_{v} \tau_{1}\right)\right)+\Omega\left(\mathscr{N}, \tau_{1}\right), \\
0=-\dot{c}\left(\Omega\left(X, \tau_{2}^{\prime}\right)+\Omega\left(\tau_{1}, \tau_{2}\right)\right)+\dot{v} \Omega\left(X, \partial_{v} \tau_{2}\right)+\Omega\left(\mathscr{N}, \tau_{2}\right) .
\end{array}\right.
$$


Так как $\tau_{2}^{\prime}=-\partial_{v} \tau_{1}$, то определитель данной системы равен

$$
D=\Omega^{2}\left(\tau_{1}, \tau_{2}\right)-\Omega\left(X, \tau_{1}^{\prime}\right) \Omega\left(X, \partial_{v} \tau_{2}\right)=\Omega^{2}\left(\tau_{1}, \tau_{2}\right)+\mathscr{O}\left(\|X\|_{E_{-\beta}}^{2}\right) .
$$

Напомним, что $\Omega\left(\tau_{1}, \tau_{2}\right) \neq 0$ в силу (3.5). Поэтому определитель $D$ не равен нулю при малых значениях $\|X\|_{E_{-\beta}}$. Решив систему (6.8), получим искомые модуляционные уравнения:

$$
\begin{aligned}
& \dot{c}=\frac{\Omega\left(\tau_{1}, \tau_{2}\right) \Omega\left(\mathscr{N}, \tau_{2}\right)+\Omega\left(X, \partial_{v} \tau_{1}\right) \Omega\left(\mathscr{N}, \tau_{2}\right)-\Omega\left(X, \partial_{v} \tau_{2}\right) \Omega\left(\mathscr{N}, \tau_{1}\right)}{D}, \\
& \dot{v}=\frac{-\Omega\left(\tau_{1}, \tau_{2}\right) \Omega\left(\mathscr{N}, \tau_{1}\right)-\Omega\left(X, \tau_{2}^{\prime}\right) \Omega\left(\mathscr{N}, \tau_{1}\right)+\Omega\left(X, \tau_{1}^{\prime}\right) \Omega\left(\mathscr{N}, \tau_{2}\right)}{D} .
\end{aligned}
$$

Из этих уравнений немедленно вытекают оценки для $\dot{c}$ и $\dot{v}$ :

$$
|\dot{v}(t)|,|\dot{c}(t)| \leqslant C_{0}(\bar{v})\|X(t)\|_{E_{-\beta}}^{2}, \quad 0 \leqslant t<t_{*},
$$

где $C_{0}(\bar{v})-$ некоторая постоянная.

\section{7. Убывание трансверсальной динамики}

В этом разделе мы докажем основное утверждение, характеризующее скорость убывания трансверсальной компоненты $X(t)$.

ПреДЛОЖЕНИЕ 7.1. Пусть выполнены все условия теоремы 2.1. Тогда $t_{*}=$ $\infty$ и, кроме того, справедливы следующие оченки:

$$
\|X(t)\|_{E_{-\beta}} \leqslant \frac{\varepsilon}{(1+|t|)^{3 / 2}}, \quad\|\Psi(t)\|_{L^{\infty}} \leqslant \frac{\varepsilon}{(1+|t|)^{1 / 2}}, \quad t \geqslant 0
$$

где в - число из определения 5.1.

Мы выведем эти оценки из уравнения (4.6) для трансверсальной компоненты $X(t)$, принимая во внимание условие ортогональности (6.1).

Отметим две главные трудности в доказательстве убывания (7.1), общие для проблем подобного рода (см., например, [7]). Во-первых, линейная часть уравнения (4.6) неавтономна, поэтому мы не можем непосредственно применить методы теории рассеяния. Аналогично подходу работы [7], сначала мы рассмотрим замороженное линейное уравнение

$$
\dot{X}(t)=A_{v_{1}} X(t), \quad 0 \leqslant t \leqslant t_{1}, \quad v_{1}=v\left(t_{1}\right),
$$

где оператор $A_{v}$ определен в $(4.15)$ и $t_{1}$ - некоторое фиксированное число из интервала $\left[0, t_{*}\right)$. Затем мы оценим возникающие погрешности. Во-вторых, даже для замороженного уравнения (7.2) убывание вида (7.1) для произвольного решения невозможно без условия ортогональности (6.1). Действительно, в силу равенств (4.16) уравнение (7.2) имеет вековые решения

$$
X(t)=C_{1} \tau_{1}(v)+C_{2}\left[\tau_{1}(v) t+\tau_{2}(v)\right]
$$


возникающие при дифференцировании солитона (2.4) по параметрам $q$ и $v$ в движущейся системе координат $y=x-v_{1} t$. Решения (7.3) принадлежат касательному пространству $\mathscr{T}_{S\left(\sigma_{1}\right)} \mathscr{S}$, где $\sigma_{1}=\left(b_{1}, v_{1}\right)\left(\right.$ с произвольным $\left.b_{1} \in \mathbb{R}\right)$, что означает неустойчивый характер нелинейной динамики вдоль солитонного многообразия. Для того чтобы исключить вековые решения, мы предполагаем выполнение условия симплектической ортогональности (6.1). Именно эти условия позволяют избавиться от растущих решений (7.3).

Обозначим через $\mathscr{X}_{v}=\mathbf{P}_{v}^{\mathrm{c}} E$ пространство, симплектически ортогональное пространству $\mathscr{T}_{S(\sigma)} \mathscr{S}$. Теперь у нас есть симплектически ортогональное разбиение

$$
\mathscr{T}_{S(\sigma)} \mathscr{E}=\mathscr{T}_{S(\sigma)} \mathscr{S}+\mathscr{X}_{v}, \quad \sigma=(b, v)
$$

и симплектическая ортогональность (6.1) может быть записана в следующем эквивалентном виде:

$$
\mathbf{P}_{v(t)}^{\mathrm{d}} X(t)=0, \quad \mathbf{P}_{v(t)}^{\mathrm{c}} X(t)=X(t), \quad 0 \leqslant t<t_{*} .
$$

Так как в силу (4.16) касательное пространство $\mathscr{T}_{S(\sigma)} \mathscr{S}$ инвариантно относительно оператора $A_{v}$, то из равенства (4.13) следует, что пространство $\mathscr{X}_{v}$ также инвариантно, т. е. $A_{v} X \in \mathscr{X}_{v}$ для плотного множества $X \in \mathscr{X}_{v}$.

7.1. Замороженная трансверсальная динамика. Зафиксируем произвольное значение $t_{1} \in\left[0, t_{*}\right)$ и запишем уравнение $(4.6)$ в "замороженном" виде

$$
\dot{X}(t)=A_{1} X(t)+\left(A(t)-A_{1}\right) X(t)+T(t)+\mathscr{N}(t), \quad 0 \leqslant t \leqslant t_{1},
$$

где $A_{1}=A_{v_{1}}$ и $v_{1}=v\left(t_{1}\right)$. Из неравенств (6.11) следует, что

$$
\|T(t)\|_{E_{\beta} \cap W} \leqslant C(\bar{v})\|X\|_{E_{-\beta}}^{2}, \quad 0 \leqslant t \leqslant t_{1},
$$

так как $w-v=\dot{c}$. Далее, из оценки (4.29) вытекает, что

$$
\|\mathscr{N}(t)\|_{E_{\beta} \cap W} \leqslant C(\bar{v})\|\Psi\|_{L^{\infty}}\|X\|_{E_{-\beta}}, \quad 0 \leqslant t \leqslant t_{1} .
$$

Следующий прием позволяет исключить “плохой" член $\left(w(t)-v_{1}\right) \nabla$ в операторе $A(t)-A_{1}$. Сделаем замену переменных $(y, t) \mapsto\left(y_{1}, t\right)=\left(y+d_{1}(t), t\right)$, где

$$
d_{1}(t):=\int_{t_{1}}^{t}\left(w(s)-v_{1}\right) d s, \quad 0 \leqslant t \leqslant t_{1} .
$$

В новых переменных $\left(y_{1}, t\right)$ уравнение (7.6) для трансверсальной компоненты принимает вид

$$
\dot{\tilde{X}}(t)=A_{1} \tilde{X}(t)+\widetilde{\mathscr{V}}(t) \widetilde{X}(t)+\widetilde{T}(t)+\widetilde{\mathscr{N}}(t), \quad 0 \leqslant t \leqslant t_{1} .
$$

Здесь

$$
\tilde{X}\left(y_{1}, t\right)=\left(\Psi\left(y_{1}-d_{1}(t), t\right), \Pi\left(y_{1}-d_{1}(t), t\right)\right), \quad \widetilde{\mathscr{V}}(t)=\mathscr{V}_{v}\left(y_{1}-d_{1}\right)-\mathscr{V}_{v_{1}}\left(y_{1}\right)
$$

и через $\widetilde{T}(t)$ и $\widetilde{\mathscr{N}}(t)$ обозначены функции $T(t)$ и $\mathscr{N}(t)$, выраженные в переменных $\left(y_{1}, t\right)$. Напомним, что матричный потенциал $\mathscr{V}_{v}$ определен в (4.25).

Получим теперь оценки для "остаточных" членов в уравнении (7.10). Для этого сначала покажем, что при $0 \leqslant t \leqslant t_{1}$ сдвиг $d_{1}(t)$ равномерно мал. 
Лемма 7.2. Для всех $t_{1}<t_{*}$ справедливо неравенство

$$
\left|d_{1}(t)\right| \leqslant C_{0}(\bar{v}) \varepsilon^{2}, \quad 0 \leqslant t \leqslant t_{1},
$$

где в-число из определения 5.1.

ДокАЗАТЕЛЬСТво. В силу (6.3)

$$
w(s)-v_{1}=w(s)-v(s)+v(s)-v_{1}=\dot{c}(s)+\int_{s}^{t_{1}} \dot{v}(\tau) d \tau .
$$

Поэтому из определений (5.4) и (7.9), а также оценок (6.11) следует, что для всех $0 \leqslant t \leqslant t_{1}$

$$
\begin{aligned}
\left|d_{1}(t)\right| & =\left|\int_{t_{1}}^{t}\left(w(s)-v_{1}\right) d s\right| \leqslant \int_{t}^{t_{1}}\left(|\dot{c}(s)|+\int_{s}^{t_{1}}|\dot{v}(\tau)| d \tau\right) d s \\
& \leqslant C_{0}(\bar{v}) m_{1}^{2}\left(t_{1}\right) \int_{t}^{t_{1}}\left(\frac{1}{(1+s)^{3}}+\int_{s}^{t_{1}} \frac{d \tau}{(1+\tau)^{3}}\right) d s \leqslant C_{0}(\bar{v}) m_{1}^{2}\left(t_{1}\right) \leqslant C_{0}(\bar{v}) \varepsilon^{2} .
\end{aligned}
$$

Лемма доказана.

Далее мы будем предполагать, что

$$
\varepsilon^{2}<\frac{\nu}{2 C_{0}(\bar{v})}
$$

где $\nu$ определено в разделе 5 . Тогда

$$
\left|d_{1}(t)\right|<\frac{\nu}{2}<1
$$

Оценим весовые нормы “сдвинутых" функций $\widetilde{T}(t)$ и $\widetilde{\mathscr{N}}(t)$ через весовые нормы функций $T(t)$ и $\mathscr{N}(t)$. Из неравенства

$$
\left(1+\left|y_{1}-d_{1}\right|\right)^{\alpha} \leqslant\left(1+\left|y_{1}\right|\right)^{\alpha}\left(1+\left|d_{1}\right|\right)^{|\alpha|} \leqslant C(\alpha)\left(1+\left|y_{1}\right|\right)^{\alpha},
$$

справедливого при всех $\alpha \in \mathbb{R}$, следует, что

$$
\|\widetilde{T}(t)\|_{E_{\beta}} \leqslant C(\beta)\|T(t)\|_{E_{\beta}}, \quad\|\widetilde{\mathscr{N}}(t)\|_{E_{\beta}} \leqslant C(\beta)\|\mathscr{N}(t)\|_{E_{\beta}} .
$$

Поэтому, используя оценки (7.7) и (7.8) для $T(t)$ и $\mathscr{N}(t)$, получим аналогичные оценки для $\widetilde{T}(t)$ и $\widetilde{\mathscr{N}}(t)$ :

$$
\begin{aligned}
\|\widetilde{T}(t)\|_{E_{\beta} \cap W} & \leqslant C(\bar{v})\|X\|_{E_{-\beta}}^{2}, \\
\|\widetilde{\mathscr{N}}(t)\|_{E_{\beta} \cap W} & \leqslant C(\bar{v})\|\Psi\|_{L^{\infty}}\|X\|_{E_{-\beta}}, \quad 0 \leqslant t \leqslant t_{1} .
\end{aligned}
$$

Наконец, оценим слагаемое $\widetilde{\mathscr{V}}(t) \widetilde{X}(t)$ в правой части уравнения (7.10). Запишем $\widetilde{\mathscr{V}}(t)$ в виде

$$
\widetilde{\mathscr{V}}(t)=\mathscr{V}_{v}\left(y_{1}-d_{1}\right)-\mathscr{V}_{v_{1}}\left(y_{1}\right)=\left(\mathscr{V}_{v}\left(y_{1}-d_{1}\right)-\mathscr{V}_{v_{1}}\left(y_{1}-d_{1}\right)\right)+\left(\mathscr{V}_{v_{1}}\left(y_{1}-d_{1}\right)-\mathscr{V}_{v_{1}}\left(y_{1}\right)\right)
$$


Аналогично (7.14) доказывается, что $\left|v(t)-v_{1}\right| \leqslant C_{0}(\bar{v}) \varepsilon^{2}$ при $0 \leqslant t \leqslant t_{1}$. Поэтому

$$
\begin{aligned}
\left|V_{v}(y)-V_{v_{1}}(y)\right| & \leqslant\left|v(t)-v_{1}\right| \max _{v \in\left[v(t), v_{1}\right]}\left|\partial_{v} V_{v}(y)\right| \\
& \leqslant C(\bar{v}) \varepsilon^{2} \max _{v \in\left[v(t), v_{1}\right]}\left|F^{\prime \prime}\left(\psi_{v}(y)\right) \partial_{v} \psi_{v}(y)\right| .
\end{aligned}
$$

Далее, из неравенства (7.12) следует, что

$$
\begin{aligned}
\left|V_{v_{1}}(y)-V_{v_{1}}\left(y_{1}\right)\right| & \leqslant\left|d_{1}(t)\right| \max _{z \in\left[y, y_{1}\right]}\left|F^{\prime \prime}\left(\psi_{v_{1}}(z)\right) \psi_{v_{1}}^{\prime}(z)\right| \\
& \leqslant C(\bar{v}) \varepsilon^{2} \max _{z \in\left[y, y_{1}\right]}\left|F^{\prime \prime}\left(\psi_{v_{1}}(z)\right) \psi_{v_{1}}^{\prime}(z)\right| .
\end{aligned}
$$

Изусловия U1, определения (4.25) и оценок (7.19)-(7.21) окончательно получим

$$
\|\widetilde{\mathscr{V}}(t) \widetilde{X}(t)\|_{E_{\beta} \cap W} \leqslant C(\bar{v}) \varepsilon^{2}\|X\|_{E_{-\beta}}, \quad 0 \leqslant t \leqslant t_{1} .
$$

7.2. Интегральные неравенства. Запишем уравнение (7.10) в интегральной форме:

$$
\widetilde{X}(t)=e^{A_{1} t} \widetilde{X}(0)+\int_{0}^{t} e^{A_{1}(t-s)}[\widetilde{\mathscr{V}}(s) \widetilde{X}(s)+\widetilde{T}(s)+\widetilde{\mathscr{N}}(s)] d s, \quad 0 \leqslant t \leqslant t_{1} .
$$

Применим симплектическую ортогональную проекцию $\mathbf{P}_{1}^{\mathrm{c}}:=\mathbf{P}_{v_{1}}^{\mathrm{c}}$ :

$$
\mathbf{P}_{1}^{\mathrm{c}} \tilde{X}(t)=e^{A_{1} t} \mathbf{P}_{1}^{\mathrm{c}} \widetilde{X}(0)+\int_{0}^{t} e^{A_{1}(t-s)} \mathbf{P}_{1}^{\mathrm{c}}[\widetilde{\mathscr{V}}(s) \widetilde{X}(s)+\widetilde{T}(s)+\widetilde{\mathscr{N}}(s)] d s, \quad 0 \leqslant t \leqslant t_{1} .
$$

Здесь мы воспользовались тем, что оператор $\mathbf{P}_{1}^{\mathrm{c}}$ коммутирует с группой $e^{A_{1} t}$. Применив неравенство (4.23), получим, что при всех $\beta>5 / 2$ и $0 \leqslant t \leqslant t_{1}$

$$
\left\|\mathbf{P}_{1}^{\mathrm{c}} \tilde{X}(t)\right\|_{E_{-\beta}} \leqslant \frac{C\|\widetilde{X}(0)\|_{E_{\beta}}}{(1+t)^{3 / 2}}+C \int_{0}^{t} \frac{\|\widetilde{\mathscr{V}}(s) \widetilde{X}(s)+\widetilde{T}(s)+\widetilde{\mathscr{N}}(s)\|_{E_{\beta}}}{(1+|t-s|)^{3 / 2}} d s .
$$

Далее, из неравенств (7.16), (7.18) и (7.22) следует, что

$$
\begin{aligned}
& \left\|\mathbf{P}_{1}^{\mathrm{c}} \tilde{X}(t)\right\|_{E_{-\beta}} \leqslant \frac{C\|X(0)\|_{E_{\beta}}}{(1+t)^{3 / 2}} \\
& \quad+C \int_{0}^{t} \frac{\varepsilon^{2}\|X(s)\|_{E_{-\beta}}+\|X(s)\|_{E_{-\beta}}^{2}+\|\Psi(s)\|_{L^{\infty}}\|X(s)\|_{E_{-\beta}}}{(1+|t-s|)^{3 / 2}} d s
\end{aligned}
$$

для всех $\beta>5 / 2$ и $0 \leqslant t \leqslant t_{1}$. Аналогично, из неравенств $(4.27),(7.16),(7.18)$ и (7.22) получим

$$
\begin{aligned}
& \left\|\left(\mathbf{P}_{1}^{\mathrm{c}} \widetilde{X}(t)\right)_{1}\right\|_{L^{\infty}} \leqslant C\left[\frac{\|X(0)\|_{E_{\beta} \cap W}}{(1+t)^{1 / 2}}\right. \\
& \left.\quad+\int_{0}^{t} \frac{\varepsilon^{2}\|X(s)\|_{E_{-\beta}}+\|X(s)\|_{E_{-\beta}}^{2}+\|\Psi(s)\|_{L^{\infty}}\|X(s)\|_{E_{-\beta}}}{(1+|t-s|)^{1 / 2}} d s\right]
\end{aligned}
$$

для всех $\beta>5 / 2$ и $0 \leqslant t \leqslant t_{1}$. 


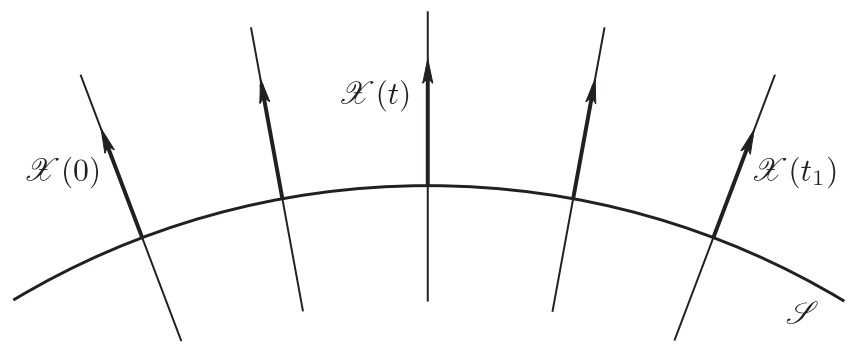

Рис. 4. Симплектическая ортогональность

7.3. Симплектическая ортогональность. Наша дальнейшая цель - заменить $\mathbf{P}_{1}^{\mathrm{c}} \tilde{X}(t)$ на $X(t)$ в левой части неравенств (7.24) и (7.25). Это можно сделать при достаточно малых $\varepsilon$, используя симплектическую ортогональность (7.5), а также тот факт, что пространства $\mathscr{X}(t):=\mathbf{P}_{v(t)}^{\mathrm{c}} E$ почти параллельны при $t \in\left[0, t_{1}\right]$ (см. рис. 4). Рассмотрим разность проекторов $\mathbf{P}_{1}^{\mathrm{d}}-\widetilde{\mathbf{P}}_{v(t)}^{\mathrm{d}}$, где

$$
\widetilde{\mathbf{P}}_{v(t)}^{\mathrm{d}} X=\sum p_{j l}(v(t)) \widetilde{\tau}_{j}(v(t)) \Omega\left(\widetilde{\tau}_{l}(v(t)), X\right), \quad X \in E,
$$

а проектор $\mathbf{P}_{1}^{\mathrm{d}}=\mathbf{P}_{v\left(t_{1}\right)}^{\mathrm{d}}$ определен в (4.22). Здесь через $\widetilde{\tau}_{j}(v(t))$ обозначены векторы $\tau_{j}(v(t))$ из $(3.3)$, выраженные в переменных $y_{1}$. Покажем, что эта разность равномерно мала по $t$ при достаточно малых $\varepsilon>0$. Так как $\tau_{j}^{\prime}$ - гладкие, быстро убывающие на бесконечности функции, то из неравенства (7.12) следует, что

$$
\left\|\widetilde{\tau}_{j}(v(t))-\tau_{j}(v(t))\right\|_{E_{\beta}} \leqslant C(\bar{v}) \varepsilon^{2}, \quad 0 \leqslant t \leqslant t_{1}, \quad j=1,2 .
$$

Далее, для всех $0 \leqslant t \leqslant t_{1}$ справедливы неравенства

$$
\begin{gathered}
\left\|\tau_{j}(v(t))-\tau_{j}\left(v\left(t_{1}\right)\right)\right\|_{E_{\beta}}=\left\|\int_{t}^{t_{1}} \dot{v}(s) \partial_{v} \tau_{j}(v(s)) d s\right\|_{E_{\beta}} \leqslant C \int_{t}^{t_{1}}|\dot{v}(s)| d s \leqslant C(\bar{v}) \varepsilon^{2}, \\
\left|p_{j l}(v(t))-p_{j l}\left(v\left(t_{1}\right)\right)\right|=\left|\int_{t}^{t_{1}} \dot{v}(s) \partial_{v} p_{j l}(v(s)) d s\right| \leqslant C \int_{t}^{t_{1}}|\dot{v}(s)| d s \leqslant C(\bar{v}) \varepsilon^{2},
\end{gathered}
$$

так как $\left|\partial_{v} p_{j l}(v(s))\right|$ равномерно ограничены в силу (5.2). Из оценок (7.27), (7.28) следует, что при достаточно малых $\varepsilon>0$

$$
\left\|\mathbf{P}_{1}^{\mathrm{d}}-\widetilde{\mathbf{P}}_{v(t)}^{\mathrm{d}}\right\|<\frac{1}{2}, \quad 0 \leqslant t \leqslant t_{1} .
$$

Так как $\widetilde{\mathbf{P}}_{v(t)}^{\mathrm{d}} \widetilde{X}(t)=0$, то из последнего неравенства немедленно вытекает, что

$$
\left\|\mathbf{P}_{1}^{\mathrm{d}} \tilde{X}(t)\right\|_{E_{-\beta}} \leqslant \frac{1}{2}\|\widetilde{X}(t)\|_{E_{-\beta}}, \quad 0 \leqslant t \leqslant t_{1} .
$$

В результате, пользуясь равенством $\mathbf{P}_{1}^{\mathrm{c}} \widetilde{X}(t)=\widetilde{X}(t)-\mathbf{P}_{1}^{\mathrm{d}} \widetilde{X}(t)$ и оценкой $(7.16)$, получим, что при достаточно малых $\varepsilon>0, t_{*}=t_{*}(\varepsilon)$ и всех $t_{1}<t_{*}$ справедливо неравенство

$$
\|X(t)\|_{E_{-\beta}} \leqslant C\|\tilde{X}(t)\|_{E_{-\beta}} \leqslant 2 C\left\|\mathbf{P}_{1}^{\mathrm{c}} \tilde{X}(t)\right\|_{E_{-\beta}}, \quad 0 \leqslant t \leqslant t_{1},
$$


где постоянная $C$ не зависит от $t_{1}$. Кроме того, из неравенства (7.29) следует, что при достаточно малых $\varepsilon>0$

$$
\left\|\left(\mathbf{P}_{1}^{\mathrm{d}} \tilde{X}(t)\right)_{1}\right\|_{L^{\infty}} \leqslant \frac{1}{2}\|\widetilde{X}(t)\|_{E_{-\beta}}, \quad 0 \leqslant t \leqslant t_{1} .
$$

Поэтому, принимая во внимание неравенство (7.30), получим

$$
\begin{aligned}
\|\Psi(t)\|_{L^{\infty}} & =\|\widetilde{\Psi}(t)\|_{L^{\infty}} \leqslant\left\|\left(\mathbf{P}_{1}^{\mathrm{c}} \tilde{X}(t)\right)_{1}\right\|_{L^{\infty}}+\left\|\left(\mathbf{P}_{1}^{\mathrm{d}} \tilde{X}(t)\right)_{1}\right\|_{L^{\infty}} \\
& \leqslant\left\|\left(\mathbf{P}_{1}^{\mathrm{c}} \widetilde{X}(t)\right)_{1}\right\|_{L^{\infty}}+\frac{1}{2}\|\widetilde{X}(t)\|_{E_{-\beta}} \\
& \leqslant\left\|\left(\mathbf{P}_{1}^{\mathrm{c}} \widetilde{X}(t)\right)_{1}\right\|_{L^{\infty}}+\left\|\mathbf{P}_{1}^{\mathrm{c}} \widetilde{X}(t)\right\|_{E_{-\beta}}, \quad 0 \leqslant t \leqslant t_{1} .
\end{aligned}
$$

7.4. Убывание трансверсальной компоненты. Теперь мы можем завершить доказательство предложения 7.1. Как уже было замечено выше, для этого достаточно доказать неравенство (5.6). Зафиксируем $\varepsilon>0$ и $t_{*}=t_{*}(\varepsilon)$, для которых справедливы оценки (7.15), (7.30) и (7.31). Теперь мы можем заменить функции $\left\|\mathbf{P}_{1}^{\mathrm{c}} \widetilde{X}(t)\right\|_{E_{-\beta}}$ и $\left\|\left(\mathbf{P}_{1}^{\mathrm{c}} \widetilde{X}(t)\right)_{1}\right\|_{L^{\infty}}$ в левой части неравенств $(7.24)$ и (7.25) на функции $\|X(t)\|_{E_{-\beta}}$ и $\|\Psi(t)\|_{L^{\infty}}$ :

$$
\begin{aligned}
&\|X(t)\|_{E_{-\beta}} \leqslant C\left[\frac{\|X(0)\|_{E_{\beta}}}{(1+t)^{3 / 2}}\right. \\
&\left.+\int_{0}^{t} \frac{\varepsilon^{2}\|X(s)\|_{E_{-\beta}}+\|X(s)\|_{E_{-\beta}}^{2}+\|\Psi(s)\|_{L^{\infty}}\|X(s)\|_{E_{-\beta}}}{(1+|t-s|)^{3 / 2}} d s\right], \\
&\|\Psi(t)\|_{L^{\infty}} \leqslant C\left[\frac{\|X(0)\|_{E_{\beta} \cap W}}{(1+t)^{1 / 2}}\right. \\
&\left.+\int_{0}^{t} \frac{\varepsilon^{2}\|X(s)\|_{E_{-\beta}}+\|X(s)\|_{E_{-\beta}}^{2}+\|\Psi(s)\|_{L^{\infty}}\|X(s)\|_{E_{-\beta}}}{(1+|t-s|)^{1 / 2}} d s\right],
\end{aligned}
$$

где $0 \leqslant t \leqslant t_{1}<t_{*}$. Выведем из этих оценок интегральные неравенства для мажорант $m_{1}$ и $m_{2}$. Умножив обе части неравенства $(7.32)$ на $(1+t)^{3 / 2}$ и взяв супремум по $t \in\left[0, t_{1}\right]$, получим

$$
\begin{aligned}
m_{1}\left(t_{1}\right) \leqslant & C\|X(0)\|_{E_{\beta}}+C \sup _{t \in\left[0, t_{1}\right]} \int_{0}^{t} \frac{(1+t)^{3 / 2} d s}{(1+|t-s|)^{3 / 2}} \\
& \times\left[\frac{\varepsilon^{2} m_{1}(s)}{(1+s)^{3 / 2}}+\frac{m_{1}^{2}(s)}{(1+s)^{3}}+\frac{m_{1}(s) m_{2}(s)}{(1+s)^{2}}\right] .
\end{aligned}
$$

Так как $m(t)$ является монотонно возрастающей функцией, то из последнего неравенства следует, что

$$
m_{1}\left(t_{1}\right) \leqslant C d_{0}+C\left[\varepsilon^{2} m_{1}\left(t_{1}\right)+m_{1}^{2}\left(t_{1}\right)+m_{1}\left(t_{1}\right) m_{2}\left(t_{1}\right)\right] I_{1}\left(t_{1}\right), \quad t_{1}<t_{*},
$$

где величина $d_{0}$ определена в $(2.8)$ и

$$
I_{1}\left(t_{1}\right)=\sup _{t \in\left[0, t_{1}\right]} \int_{0}^{t} \frac{(1+t)^{3 / 2}}{(1+|t-s|)^{3 / 2}} \frac{d s}{(1+s)^{3 / 2}} .
$$


Разделив последний интеграл на два интеграла по отрезкам $0 \leqslant s \leqslant t / 2$ и $t / 2 \leqslant s \leqslant t$, легко проверить, что этот интеграл оценивается константой, не зависящей от $t$. Поэтому из (7.34) следует, что существует константа $C$, не зависящая от $t_{1}$, такая, что

$$
m_{1}\left(t_{1}\right) \leqslant C d_{0}+C\left[\varepsilon^{2} m_{1}\left(t_{1}\right)+m_{1}^{2}\left(t_{1}\right)+m_{1}\left(t_{1}\right) m_{2}\left(t_{1}\right)\right], \quad t_{1}<t_{*} .
$$

Аналогично, умножая обе части неравенства $(7.33)$ на $(1+t)^{1 / 2}$, получим

$$
m_{2}\left(t_{1}\right) \leqslant C d_{0}+C\left[\varepsilon^{2} m_{1}\left(t_{1}\right)+m_{1}^{2}\left(t_{1}\right)+m_{1}\left(t_{1}\right) m_{2}\left(t_{1}\right)\right], \quad t_{1}<t_{*},
$$

где константа $C$ не зависит от $t_{1}$. Обозначим через $M(t)$ вектор с компонентами $m_{1}(t)$ и $m_{2}(t)$. Из неравенств $(7.35)$ и (7.36) следует, что

$$
\left|M\left(t_{1}\right)\right| \leqslant C d_{0}+C\left[\varepsilon^{2}\left|M\left(t_{1}\right)\right|+\left|M\left(t_{1}\right)\right|^{2}\right], \quad t_{1}<t_{*} .
$$

Так как $m_{i}(t)<\varepsilon$ в силу $(5.5)$, то функция $M\left(t_{1}\right)$ ограничена при достаточно малых $d_{0}$ и $\varepsilon$ :

$$
\left|M\left(t_{1}\right)\right| \leqslant C d_{0}, \quad t_{1}<t_{*}
$$

где константа $C=C(\bar{v})$ не зависит от $t_{*}$. Выберем $d_{0}$ в $(2.8)$ настолько малым, чтобы выполнялось неравенство $d_{0}<\varepsilon /(2 C)$. Тогда из $(7.37)$ немедленно вытекают неравенства (5.6) для мажорант $m_{j}$. Далее, из неравенств $(6.11),(7.1)$ и (7.15) следует, что

$$
|v(t)-v(0)| \leqslant \int_{0}^{t}|\dot{v}(s)| d s \leqslant B(\bar{v}) \varepsilon^{2}<\frac{\nu}{2}, \quad 0 \leqslant t<t_{*},
$$

т. е. первое неравенство (5.6) тоже выполнено. Поэтому $t_{*}=\infty$, и оценка (7.37) справедлива для всех $t_{1}>0$.

\section{8. Солитонная асимптотика}

Выведем главную теорему 2.1 из неравенства (7.1). Из неравенств (6.11) и (7.1) следует, что при всех $t \geqslant 0$ справедлива оценка

$$
|\dot{c}(t)|+|\dot{v}(t)| \leqslant C\left(\bar{v}, d_{0}\right)(1+t)^{-3} .
$$

Поэтому $c(t)=c_{+}+\mathscr{O}\left(t^{-2}\right)$ и $v(t)=v_{+}+\mathscr{O}\left(t^{-2}\right)$ при $t \rightarrow \infty$, и, следовательно,

$$
b(t)=c(t)+\int_{0}^{t} v(s) d s=v_{+} t+q_{+}+\mathscr{O}\left(t^{-1}\right), \quad t \rightarrow \infty,
$$

где $c_{+}, v_{+}$и $q_{+}-$некоторые константы. Запишем решение $Y(x, t)$ уравнения (2.1) в виде

$$
Y(x, t)=Y_{v(t)}(x-b(t), t)+X(x-b(t), t) .
$$


Так как $\left\|Y_{v(t)}(x-b(t), t)-Y_{v_{+}}\left(x-v_{+} t-q_{+}, t\right)\right\|_{E}=\mathscr{O}\left(t^{-1}\right)$, то для получения асимптотики (2.9) с остаточным членом $(2.10)$, имеющим порядок $t^{-1 / 2}$, достаточно извлечь дисперсионную волну $W_{0}(t) \Phi_{+}$из слагаемого $X(x-b(t), t)$. Подставляя равенство (8.3) в (2.1) и используя уравнения (4.4), получим следующее неоднородное уравнение для векторной функции $X(x-b(t), t)=(\Psi(x-$ $b(t), t), \Pi(x-b(t), t)):$

$$
\dot{X}(y, t)=A_{v}^{0} X(y, t)+R(y, t), \quad y=x-b(t),
$$

где

$$
A_{v}^{0}=\left(\begin{array}{cc}
v \nabla & 1 \\
\Delta-m^{2} & v \nabla
\end{array}\right), \quad R(t)=\left(\begin{array}{c}
-\dot{v} \partial_{v} \psi_{v} \\
-\dot{v} \partial_{v} \pi_{v}-V_{v} \Psi(t)+N(v, \Psi(t))
\end{array}\right)
$$

Уравнение (8.4) в переменной $x=y+b(t)$ имеет вид

$$
\dot{\widetilde{X}}(t)=A_{0}^{0} \widetilde{X}(t)+\widetilde{R}(t), \quad t \geqslant 0,
$$

где $\widetilde{X}(x, t)=X(x-b(t), t), \widetilde{R}(x, t)=R(x-b(t), t)$ и $A_{0}^{0}-$ это оператор $A_{v}^{0}$, соответствующий $v=0$. Следовательно,

$$
\begin{aligned}
\widetilde{X}(t) & =W_{0}(t) \widetilde{X}(0)+\int_{0}^{t} W_{0}(t-s) \widetilde{R}(s) d s \\
& =W_{0}(t)\left(\widetilde{X}(0)+\int_{0}^{\infty} W_{0}(-s) \widetilde{R}(s) d s\right)-\int_{t}^{\infty} W_{0}(t-s) \widetilde{R}(s) d s \\
& =W_{0}(t) \Phi_{+}+r_{+}(t),
\end{aligned}
$$

где $W_{0}(t)$ - динамическая группа свободного уравнения Клейна-Гордона. Для того чтобы установить асимптотику (2.9), достаточно проверить, что

$$
\begin{aligned}
\Phi_{+} & =\widetilde{X}(0)+\int_{0}^{\infty} W_{0}(-s) \widetilde{R}(s) d s \in E \\
\left\|r_{+}(t)\right\|_{E} & =\left\|\int_{t}^{\infty} W_{0}(t-s) \widetilde{R}(s) d s\right\|_{E}=\mathscr{O}\left(t^{-1 / 2}\right) .
\end{aligned}
$$

Из условия $(2.8)$ следует, что $\widetilde{X}(0) \in E$. Представим $\widetilde{R}(s)$ в виде суммы:

$$
\widetilde{R}(s)=\left(\begin{array}{l}
-\dot{v} \partial_{v} \widetilde{\psi}_{v} \\
-\dot{v} \partial_{v} \widetilde{\pi}_{v}
\end{array}\right)+\left(\begin{array}{c}
0 \\
-\widetilde{V}_{v} \widetilde{\Psi}(s)+\widetilde{N}(v, \widetilde{\Psi}(s))
\end{array}\right)=\widetilde{R}^{\prime}(s)+\widetilde{R}^{\prime \prime}(s) .
$$

В силу неравенства (8.1)

$$
\left\|\widetilde{R}^{\prime}(s)\right\|_{E}=\left\|R^{\prime}(s)\right\|_{E}=\mathscr{O}\left(s^{-3}\right) .
$$

Далее, из оценок (7.1) следует, что

$$
\left\|\widetilde{V}_{v} \widetilde{\Psi}(s)\right\|_{L^{2}}=\left\|V_{v} \Psi(s)\right\|_{L^{2}} \leqslant C\|\Psi(s)\|_{L_{-\beta}^{2}} \leqslant C\left(\bar{v}, d_{0}\right)(1+|s|)^{-3 / 2},
$$


так как потенциал $V_{v}$ имеет компактный носитель. Аналогично, из оценок (7.1) и (7.8) следует, что

$$
\|\tilde{N}(v, \widetilde{\Psi}(s))\|_{L^{2}}=\|N(v, \Psi(s))\|_{L^{2}} \leqslant C\left(\bar{v}, d_{0}\right)(1+|s|)^{-3 / 2} .
$$

Из двух последних неравенств получим $\left\|\widetilde{R}^{\prime \prime}(s)\right\|_{E}=\mathscr{O}\left(s^{-3 / 2}\right)$. Вместе с (8.6) это означает, что $\|\widetilde{R}(s)\|_{E}=\mathscr{O}\left(s^{-3 / 2}\right)$ и, следовательно, интегралы в $(8.5)$ сходятся в силу "унитарности" группы $W_{0}(t)$ в пространстве $E$. Асимптотика $(8.5)$ для $r_{+}(t)$ доказывается аналогично.

\section{Глава II. Стоячий солитон}

В этой главе мы доказываем асимптотическую устойчивость кинков в более сложной спектральной ситуации: при наличии дополнительного дискретного спектра линеаризованной динамики. Для простоты изложения мы рассматриваем "стоячий кинк", т. е. кинк с нулевой скоростью $v=0$, и его нечетные возмущения.

\section{9. Формулировка главного результата}

Итак, мы рассматриваем только нечетные решения $Y(-x, t)=Y(x, t)$. Пространство нечетных состояний инвариантно относительно динамической группы уравнения (2.3), так как согласно условию $\mathbf{U 1}$ потенциал $U(\psi)$ является четной функцией и, следовательно, функция $F(\psi)$ нечетна. Главным результатом является следующая теорема.

ТеОрема 9.1. Пустъ выполнены условия U1-U3, D2 и F, и пусть $Y(t)$ является решением задачи Коши (2.3) с нечетными начальными данными $Y_{0} \in \mathscr{E}$, достаточно близкими к кинку:

$$
Y_{0}=(s(x), 0)+X_{0}, \quad d_{0}:=\left\|X_{0}\right\|_{E_{\beta} \cap W} \ll 1,
$$

где $\beta>5 / 2$. Тогда справедлива следующая асимптотика:

$$
Y(x, t)=(s(x), 0)+W_{0}(t) \Phi_{ \pm}+r_{ \pm}(x, t), \quad t \rightarrow \pm \infty,
$$

где $\Phi_{ \pm} \in E$ - асимптотические состояния рассеяния, $W_{0}(t)$ - динамическая группа свободного уравнения Клейна-Гордона. Кроме того,

$$
\left\|r_{ \pm}(t)\right\|_{E}=\mathscr{O}\left(|t|^{-1 / 3}\right), \quad t \rightarrow \pm \infty
$$

\section{0. Линеаризация на солитоне}

Разлагая решения уравнения (2.3) в сумму $Y(t)=S+X(t)$, где $S=(s, 0)$, получим уравнение для $X(t)$ :

$$
\dot{X}(t)=A X(t)+\mathscr{N}(X(t)), \quad t \in \mathbb{R},
$$


где

$$
A=\left(\begin{array}{cc}
0 & 1 \\
-\Delta+m^{2}+V(x) & 0
\end{array}\right), \quad V(x)=-F^{\prime}(s(x))-m^{2}=U^{\prime \prime}(s(x))-m^{2} .
$$

Нелинейная часть $\mathscr{N}(X)$ выглядит следующим образом:

$$
\mathscr{N}(X)=\left(\begin{array}{c}
0 \\
N(\Psi)
\end{array}\right), \quad N(\Psi)=F(s+\Psi)-F(s)-F^{\prime}(s) \Psi .
$$

Непрерывный спектр оператора $A$ совпадает с интервалом $\Gamma:=(-i \infty,-i m] \cup$ $[i m, i \infty)$. Концевые точки $\pm i m$ непрерывного спектра не являются ни собственными значениями, ни резонансами оператора $A$ в силу условия U3. Найдем дискретный спектр оператора $A$. Для этого рассмотрим спектральное уравнение

$$
A\left(\begin{array}{l}
u_{1} \\
u_{2}
\end{array}\right)=\left(\begin{array}{cc}
0 & 1 \\
-H & 0
\end{array}\right)\left(\begin{array}{l}
u_{1} \\
u_{2}
\end{array}\right)=\Lambda\left(\begin{array}{l}
u_{1} \\
u_{2}
\end{array}\right)
$$

где $u=\left(u_{1}, u_{2}\right) \in L^{2} \oplus L^{2}$. Из первого уравнения найдем $u_{2}=\Lambda u_{1}$. Подставив во второе уравнение, получим $\left(H+\Lambda^{2}\right) u_{1}=0$. Из условия $\mathbf{D} 2$ следует, что $\Lambda^{2}$ может принимать только одно значение: $\Lambda^{2}=-\lambda_{1}$. Поэтому оператор $A$ на подпространстве нечетных функций имеет два чисто мнимых собственных значения $\Lambda= \pm i \mu$, где $\mu=\sqrt{\lambda_{1}}$. Соответствующие собственные функции имеют вид

$$
u=\left(\begin{array}{c}
u_{1} \\
u_{2}
\end{array}\right)=\left(\begin{array}{c}
\varphi_{\lambda_{1}} \\
i \mu \varphi_{\lambda_{1}}
\end{array}\right), \quad \bar{u}=\left(\begin{array}{c}
\varphi_{\lambda_{1}} \\
-i \mu \varphi_{\lambda_{1}}
\end{array}\right),
$$

где $\varphi_{\lambda_{1}}$ - собственная функция оператора $H$, соответствующая собственному значению $\lambda_{1}$. Заметим, что мы можем выбрать функцию $\varphi_{\lambda_{1}}$ вещественной, так как дифференциальный оператор $H$ имеет вещественные коэффициенты.

Убывание линеаризованной динамики. Рассмотрим линеаризованное уравнение

$$
\dot{X}(t)=A X(t), \quad t \in \mathbb{R} .
$$

Обозначим через $\langle\cdot, \cdot\rangle$ скалярное произведение в пространстве $L^{2}\left(\mathbb{R}, \mathbb{C}^{2}\right)$. Определим симплектический проектор $P^{\mathrm{d}}$ на собственное подпространство $\mathscr{X}^{\mathrm{d}}$, порожденное собственными функциями $u$ и $\bar{u}$ :

$$
P^{\mathrm{d}} X=\frac{\langle X, j u\rangle}{\langle u, j u\rangle} u+\frac{\langle X, j \bar{u}\rangle}{\langle\bar{u}, j \bar{u}\rangle} \bar{u}, \quad j=\left(\begin{array}{cc}
0 & -1 \\
1 & 0
\end{array}\right) .
$$

Заметим, что если функция $X$ вещественна, то ее проекция $P^{\mathrm{d}} X$ также вещественна. Обозначим через $\mathscr{X}^{\mathrm{c}}$ подпространство непрерывного спектра оператора $A$ и через $P^{\mathrm{c}}=1-P^{\mathrm{d}}$ проектор на это подпространство. Для оператора $A$ справедливы оценки, аналогичные оценкам (4.23) и (4.27) из первой части. А именно: для любого $\beta>5 / 2$

$$
\begin{array}{rlrl}
\left\|e^{A t} P^{\mathrm{c}} X\right\|_{E_{-\beta}} \leqslant C(1+t)^{-3 / 2}\|X\|_{E_{\beta}}, & & t \in \mathbb{R}, \\
\left\|\left(e^{A t} P^{\mathrm{c}} X\right)_{1}\right\|_{L^{\infty}} \leqslant C(1+t)^{-1 / 2}\left(\|X\|_{W}+\|X\|_{E_{\beta}}\right), & t \in \mathbb{R} .
\end{array}
$$


Мы будем использовать еще одну оценку, доказательство которой можно найти в [28]:

$$
\left\|e^{A t}(A \mp 2 i \mu-0)^{-1} P^{\mathrm{c}} X\right\|_{E_{-\beta}} \leqslant C(1+t)^{-3 / 2}\|X\|_{E_{\beta}}, \quad \beta>\frac{5}{2}, \quad t \in \mathbb{R} .
$$

\section{1. Асимптотическое разложение динамики}

Будем искать решение уравнения (10.1) в виде суммы $X(t):=w(t)+f(t)$, где функция $w(t)=z(t) u+\bar{z}(t) \bar{u}$ принадлежит пространству $\mathscr{X}^{\mathrm{d}}$, а функция $f(t)$ - пространству $\mathscr{X}^{\mathrm{c}}$. Выведем динамические уравнения для функций $z(t)$ и $f(t)$.

Применим проектор $P^{\mathrm{d}}$ к обеим частям уравнения (10.1):

$$
\dot{z} u+\dot{\bar{z}} \bar{u}=A w+P^{\mathrm{d}} \mathscr{N} .
$$

Так как $\langle\bar{u}, j u\rangle=0, A w=i \mu(z u-\bar{z} \bar{u})$ и $\left(P^{\mathrm{d}}\right)^{*} j=j P^{\mathrm{d}}$, то, умножая скалярно уравнение (11.1) на $j u$, получим

$$
(\dot{z}-i \mu z)\langle u, j u\rangle=\langle\mathscr{N}, j u\rangle .
$$

Применяя проектор $P^{\mathrm{c}}$ к обеим частям (10.1), получим уравнение для $f(t)$ :

$$
\dot{f}=A f+P^{\mathrm{c}} \mathscr{N} .
$$

ЗАмЕчАниЕ 11.1. Ниже мы докажем следующие асимптотики для функций $z(t)$ и $f(t)$ :

$$
\|f(t)\|_{E_{-\beta}} \sim t^{-1}, \quad z(t) \sim t^{-1 / 2}, \quad\left\|f_{1}(t)\right\|_{L^{\infty}} \sim t^{-1 / 2}, \quad t \rightarrow \infty .
$$

Используя эти асимптотики, разложим правую часть уравнения (11.2) до членов порядка $\mathscr{O}\left(t^{-3 / 2}\right)$, а правую часть уравнения $(11.3)$ до членов порядка $\mathscr{O}\left(t^{-1}\right)$ и затем установим асимптотики (11.4) с помощью метода мажорант.

Для начала разложим нелинейный член $N(x, \Psi)$, определенный в $(10.3)$, в ряд Тейлора

$$
N(x, \Psi)=N_{2}(x, \Psi)+N_{R}(x, \Psi)
$$

где

$$
N_{2}(x, \Psi)=\frac{F^{\prime \prime}(s(x))}{2} \Psi^{2}(x), \quad N_{R}(x, \Psi)=\frac{\Psi^{3}(x)}{3 !} \int_{0}^{1}(1-\rho)^{2} F^{\prime \prime \prime}(s(x)+\rho \Psi(x)) d \rho .
$$

По условию $\mathbf{U 2}$ функция $F^{\prime \prime \prime}(\psi)$ равна нулю в некоторой окрестности точек $\pm a$. Поэтому $N_{R}(x, \Psi)=0$ при больших значениях $x$ и, следовательно, справедлива следующая оценка:

$$
\begin{aligned}
\left\|N_{R}\right\|_{L_{\beta}^{2} \cap W_{0}^{1,1}}=\mathscr{R} & \left(|z|+\left\|f_{1}\right\|_{L^{\infty}}\right)\left(|z|^{3}+|z|^{2}\|f\|_{E_{-\beta}}\right. \\
& \left.+|z|\left\|f_{1}\right\|_{L^{\infty}}\|f\|_{E_{-\beta}}+\left\|f_{1}\right\|_{L^{\infty}}^{2}\|f\|_{E_{-\beta}}\right) .
\end{aligned}
$$

Определим также симметричные билинейную $\mathscr{N}_{2}\left[X_{1}, X_{2}\right]=\left(0, N_{2}\left[\Psi_{1}, \Psi_{2}\right]\right)$ и трилинейную $\mathscr{N}_{3}\left[X_{1}, X_{2}, X_{3}\right]=\left(0, N_{3}\left[\Psi_{1}, \Psi_{2}, \Psi_{3}\right]\right)$ формы, где

$$
N_{2}\left[\Psi_{1}, \Psi_{2}\right]=\frac{F^{\prime \prime}(s)}{2} \Psi_{1} \Psi_{2}, \quad N_{3}\left[\Psi_{1}, \Psi_{2}, \Psi_{3}\right]=\frac{F^{\prime \prime \prime}(s)}{6} \Psi_{1} \Psi_{2} \Psi_{3} .
$$


11.1. Асимптотическое разложение $\dot{z}$. Перепишем уравнение (11.2) следующим образом:

$$
\dot{z}-i \mu z=\frac{\langle\mathscr{N}, j u\rangle}{\langle u, j u\rangle}=\frac{\left\langle\mathscr{N}_{2}[w, w]+2 \mathscr{N}_{2}[w, f]+\mathscr{N}_{3}[w, w, w], j u\right\rangle}{\langle u, j u\rangle}+Z_{R},
$$

где

$$
\left|Z_{R}\right|=\mathscr{R}\left(|z|+\left\|f_{1}\right\|_{L^{\infty}}\right)\left(|z|^{2}+\|f\|_{E_{-\beta}}\right)^{2}
$$

Так как

$\mathscr{N}_{2}[w, w]=\left(z^{2}+2 z \bar{z}+\bar{z}^{2}\right) \mathscr{N}_{2}[u, u], \quad \mathscr{N}_{3}[w, w, w]=\left(z^{3}+3 z^{2} \bar{z}+3 z \bar{z}^{2}+\bar{z}^{3}\right) \mathscr{N}_{3}[u, u, u]$,

то из (11.8) следует, что

$$
\dot{z}=i \mu z+Z_{2}\left(z^{2}+2 z \bar{z}+\bar{z}^{2}\right)+Z_{3}\left(z^{3}+3 z^{2} \bar{z}+3 z \bar{z}^{2}+\bar{z}^{3}\right)+(z+\bar{z})\left\langle f, j Z_{1}^{\prime}\right\rangle+Z_{R},
$$

где

$$
Z_{2}=\frac{\left\langle\mathscr{N}_{2}[u, u], j u\right\rangle}{\langle u, j u\rangle}, \quad Z_{3}=\frac{\left\langle\mathscr{N}_{3}[u, u, u], j u\right\rangle}{\langle u, j u\rangle}, \quad Z_{1}^{\prime}=2 \frac{\mathscr{N}_{2}[u, u]}{\langle u, j u\rangle}
$$

согласно (11.7). Заметим, что из (10.4) следует, что число $\langle u, j u\rangle$ является чисто мнимым:

$$
\langle u, j u\rangle=2 i \mu\left|\varphi_{\lambda_{1}}\right|^{2}=i \delta, \quad \text { где } \delta=2 \mu\left|\varphi_{\lambda_{1}}\right|^{2}>0 .
$$

Следовательно, коэффициенты $Z_{2}, Z_{3}$ и $Z_{1}^{\prime}$ тоже чисто мнимые.

11.2. Асимптотическое разложение $\dot{f}$. Перепишем уравнение (11.3) следующим образом:

$$
\dot{f}=A f+P^{\mathrm{c}} \mathscr{N}=A f+P^{\mathrm{c}} \mathscr{N}_{2}[w, w]+F_{R}
$$

Остаточный член $F_{R}=F_{R}(x, t)$ имеет вид

$$
F_{R}=P^{\mathrm{c}}\left(\mathscr{N}_{R}-2 \mathscr{N}_{2}[f, w]-\mathscr{N}_{2}[f, f]\right)=\left(1-P^{\mathrm{d}}\right)\left(\mathscr{N}_{R}-2 \mathscr{N}_{2}[f, w]-\mathscr{N}_{2}[f, f]\right),
$$

где $\mathscr{N}_{R}=\left(0, N_{R}\right)$ и $N_{R}$ определено в (11.5). Из оценки (11.6) следует, что

$$
\left\|F_{R}\right\|_{E_{\beta} \cap W}=\mathscr{R}\left(|z|+\left\|f_{1}\right\|_{L^{\infty}}\right)\left(|z|^{3}+|z|\|f\|_{E_{-\beta}}+\left\|f_{1}\right\|_{L^{\infty}}\|f\|_{E_{-\beta}}\right) .
$$

\section{2. Нормальные формы Пуанкаре}

В этом разделе мы избавимся от "нерезонансных" членов в уравнениях (11.8) и (11.14) и получим так называемые "нормальные формы" Пуанкаре для этих уравнений. 
12.1. Нормальная форма для $\dot{f}$. Запишем уравнение (11.14) более подробно:

$$
\dot{f}=A f+\left(z^{2}+2 z \bar{z}+\bar{z}^{2}\right) F_{2}+F_{R}, \quad F_{2}=P^{\mathrm{c}} \mathscr{N}_{2}[u, u] .
$$

Выделим в этом уравнении члены порядка $z^{2} \sim t^{-1}$. Для этого представим функцию $f$ как сумму

$$
f=h+k+g,
$$

где

$$
k=a_{20} z^{2}+2 a_{11} z \bar{z}+a_{02} \bar{z}^{2}
$$

с некоторыми коэффициентами $a_{i j}(x)$, удовлетворяющими условию $a_{j i}(x)=$ $\bar{a}_{i j}(x)$, и

$$
g(t)=-e^{A t} k(0)
$$

Заметим, что $h(0)=f(0)$.

Лемма 12.1. Существуют функиии $a_{i j}$, принадлежсащие пространству $E_{-\beta}$, такие, что функиия $h=f-k-g$ удовлетворяет уравнению

$$
\dot{h}=A h+H_{R},
$$

где

$$
H_{R}=F_{R}+H_{I}, \quad H_{I}=\sum a_{i j}(x) \mathscr{R}\left(|z|+\left\|f_{1}\right\|_{L^{\infty}}\right)|z|\left(|z|+\|f\|_{E_{-\beta}}\right)^{2} .
$$

ДокАзАтЕльство. Подставляя равенства (12.3), (12.4) в уравнение (12.1), получим

$$
\begin{aligned}
\dot{h}=\dot{f} & -\left(2 a_{20} z+2 a_{11} \bar{z}\right) \dot{z}-\left(2 a_{11} z+2 a_{02} \bar{z}\right) \dot{\bar{z}}-\dot{g} \\
=A & +\left(z^{2}+2 z \bar{z}+\bar{z}^{2}\right) F_{2}+F_{R} \\
& -\left(2 a_{20} z+2 a_{11} \bar{z}\right)\left(i \mu z+\mathscr{R}\left(|z|+\|f\|_{L^{\infty}}\right)\left(|z|+\|f\|_{E_{-\beta}}\right)^{2}\right) \\
& -\left(2 a_{11} z+2 a_{02} \bar{z}\right)\left(-i \mu \bar{z}+\mathscr{R}\left(|z|+\left\|f_{1}\right\|_{L^{\infty}}\right)\left(|z|+\|f\|_{E_{-\beta}}\right)^{2}\right)-A g .
\end{aligned}
$$

С другой стороны, (12.5) означает, что

$$
\dot{h}=A\left(f-a_{20} z^{2}-2 a_{11} z \bar{z}-a_{02} \bar{z}^{2}-g\right)+H_{R} .
$$

Сравнивая коэффициенты при $z^{2}, z \bar{z}$ и $\bar{z}^{2}$ в правых частях равенств (12.6) и (12.7), получим

$$
F_{2}-2 i \mu a_{20}=-A a_{20}, \quad F_{2}=-A a_{11}, \quad F_{2}+2 i \mu a_{02}=-A a_{02} .
$$

Так как оператор $A: H^{s_{1}+2} \oplus H^{s_{2}} \rightarrow H^{s_{2}} \oplus H^{s_{1}}$ эллиптический и не имеет ядра в пространстве нечетных функций, то существует обратный непрерывный оператор $A^{-1}: H^{s_{2}} \oplus H^{s_{1}} \rightarrow H^{s_{1}+2} \oplus H^{s_{2}}$, и из второго уравнения (12.8) найдем

$$
a_{11}=-A^{-1} F_{2}
$$

где $F_{2}=P^{\mathrm{c}} \mathscr{N}_{2}[u, u] \in H^{s_{2}} \oplus H^{s_{1}}$ для любых $s_{1}, s_{2}>0$. Далее, найдем коэффициенты $a_{20}$ и $a_{02}$ из первого и третьего уравнений (12.8):

$$
a_{20}=-(A+2 i \mu-0)^{-1} F_{2}, \quad a_{02}=\bar{a}_{20}=-(A-2 i \mu-0)^{-1} F_{2} .
$$


Как будет показано ниже, такой выбор обратных операторов обеспечивает оценки (10.8). Так как точки $\pm 2 i \mu$ принадлежат непрерывному спектру, то, согласно принципу предельного поглощения (см. [1], [19]), эти обратные операторы существуют и действуют непрерывно из пространства $E_{\sigma}$ в пространство $E_{-\sigma}$ с любым $\sigma>1 / 2$. Лемма доказана.

Слагаемое $H_{I}$ в остаточном члене $H_{R}$ может быть записано как

$$
H_{I}=\sum_{n}(A-2 i \mu n-0)^{-1} C_{n}, \quad n \in\{-1,0,1\},
$$

где функции $C_{n} \in E^{\mathrm{c}}$ удовлетворяют оценке

$$
\left\|C_{n}\right\|_{E_{\beta}}=\mathscr{R}\left(|z|+\|f\|_{E_{-\beta}}\right)|z|\left(|z|+\|f\|_{E_{-\beta}}\right)^{2} .
$$

12.2. Нормальная форма для $\dot{z}$. Подставляя разложение (12.2) в уравнение (11.11) для $z$, получим

$$
\begin{aligned}
\dot{z}=i \mu z & +Z_{2}\left(z^{2}+2 z \bar{z}+\bar{z}^{2}\right)+Z_{3}\left(z^{3}+3 z^{2} \bar{z}+3 z \bar{z}^{2}+\bar{z}^{3}\right) \\
& +Z_{30}^{\prime} z^{3}+Z_{21}^{\prime} z^{2} \bar{z}+Z_{12}^{\prime} z \bar{z}^{2}+Z_{03}^{\prime} \bar{z}^{3}+\widetilde{Z}_{R},
\end{aligned}
$$

где

$$
\begin{array}{ll}
Z_{30}^{\prime}=\left\langle a_{20}, j Z_{1}^{\prime}\right\rangle, & Z_{21}^{\prime}=\left\langle a_{11}+a_{20}, j Z_{1}^{\prime}\right\rangle, \\
Z_{03}^{\prime}=\left\langle a_{02}, j Z_{1}^{\prime}\right\rangle, & Z_{12}^{\prime}=\left\langle a_{02}+a_{11}, j Z_{1}^{\prime}\right\rangle .
\end{array}
$$

Новый остаточный член $\widetilde{Z}_{R}$ имеет вид $Z_{R}+(z+\bar{z})\left\langle f-k, j Z_{1}^{\prime}\right\rangle$, где $Z_{R}$ удовлетворяет оценке (11.9). Так как $f-k=g+h$, то

$$
\left|\left\langle f-k, Z_{1}^{\prime}\right\rangle\right| \leqslant C\left(\|g\|_{E_{-\beta}}+\|h\|_{E_{-\beta}}\right) .
$$

Отсюда и из (11.9) вытекает, что

$$
\left|\widetilde{Z}_{R}\right|=\mathscr{R}_{1}\left(|z|+\|f\|_{L^{\infty}}\right)\left[\left(|z|^{2}+\|f\|_{E_{-\beta}}\right)^{2}+|z|\|g\|_{E_{-\beta}}+|z|\|h\|_{E_{-\beta}}\right] .
$$

Особую важную роль в уравнении (12.13) играют резонансные члены, содержащие $z^{2} \bar{z}=|z|^{2} z$. А именно, метод нормальных форм Пуанкаре позволяет исключить все полиномиальные члены в правой части в (12.13), кроме первого и резонансного.

Покажем, что при выполнении условия невырожденности $\mathbf{F}$ вещественная часть коэффициента $Z_{21}^{\prime}$ строго отрицательна. Из формул (11.12), (12.9) и (12.10) следует, что

$$
\begin{aligned}
Z_{21}^{\prime}= & -\left\langle A^{-1} P^{\mathrm{c}} \mathscr{N}_{2}[u, u], 2 j \frac{\mathscr{N}_{2}[u, u]}{\langle u, j u\rangle}\right\rangle \\
& -\left\langle(A-2 i \mu-0)^{-1} P^{\mathrm{c}} \mathscr{N}_{2}[u, u], 2 j \frac{\mathscr{N}_{2}[u, u]}{\langle u, j u\rangle}\right\rangle .
\end{aligned}
$$

Число $\left\langle A^{-1} P^{c} j \mathscr{N}_{2}[u, u], 2 \mathscr{N}_{2}[u, u]\right\rangle$ вещественно в силу самосопряженности оператора $A^{-1} P^{\mathrm{c}} j$, поэтому первое слагаемое в правой части равенства $(12.16)$ 
чисто мнимое. Следовательно,

$$
\begin{aligned}
\operatorname{Re} Z_{21}^{\prime} & =2 \operatorname{Re} \frac{\left\langle(A-2 i \mu-0)^{-1} P^{\mathrm{c}} \mathscr{N}_{2}[u, u], j \mathscr{N}_{2}[u, u]\right\rangle}{i \delta} \\
& =\frac{2}{\delta} \operatorname{Im}\left\langle R(2 i \mu+0) P^{\mathrm{c}} \mathscr{N}_{2}[u, u], j \mathscr{N}_{2}[u, u]\right\rangle,
\end{aligned}
$$

где $R(\lambda)=(A-\lambda)^{-1}, \operatorname{Re} \lambda>0,-$ резольвента оператора $A$. Так как проектор $P^{\mathrm{c}}$ коммутирует с резольвентой, то

$$
R(2 i \mu+0) P^{\mathrm{c}}=P^{\mathrm{c}} R(2 i \mu+0) P^{\mathrm{c}} .
$$

Кроме того, $\left(P^{\mathrm{c}}\right)^{*} j=j P^{\mathrm{c}}$. Следовательно,

$$
\operatorname{Re} Z_{21}^{\prime}=\frac{2}{\delta} \operatorname{Im}\langle R(2 i \mu+0) \alpha, j \alpha\rangle, \quad \alpha=P^{\mathrm{c}} \mathscr{N}_{2}[u, u] .
$$

Теперь воспользуемся следующим спектральным представлением (см. [7; формула (2.1.9)]):

$$
\begin{aligned}
& \langle R(2 i \mu+0) \alpha, j \alpha\rangle \\
& \quad=\frac{1}{i} \int_{m}^{\infty} \theta(\lambda) d \lambda\left(\frac{\langle\alpha, j u(i \lambda)\rangle\langle u(i \lambda), j \alpha\rangle}{i \lambda-2 i \mu-0}+\frac{\langle\alpha, j \bar{u}(i \lambda)\rangle\langle\bar{u}(i \lambda), j \alpha\rangle}{-i \lambda-2 i \mu-0}\right) \\
& \quad=\int_{m}^{\infty} \theta(\lambda) d \lambda\left(\frac{\langle u(i \lambda), j \alpha\rangle \overline{\langle u(i \lambda), j \alpha\rangle}}{\lambda-2 \mu+i 0}+\frac{\langle\bar{u}(i \lambda), j \alpha\rangle \overline{\langle(i \lambda), j \alpha\rangle}}{\lambda+2 \mu-i 0}\right)
\end{aligned}
$$

где $\theta(\lambda)=1 /\left(2 \pi N^{2}(\lambda) \sqrt{\lambda-m}\right)$ и $N(\lambda)$ - некоторый вещественный нормализующий множитель. Принимая во внимание равенство $1 /(\nu+i 0)=\operatorname{p.v} .(1 / \nu)-$ $i \pi \delta(\nu)$, где p.v. означает главное значение по Коши, получим

$$
\begin{aligned}
\langle R(2 i \mu+0) \alpha, j \alpha\rangle= & \int_{m}^{\infty} \theta(\lambda) d \lambda\left(\frac{|\langle u(i \lambda), j \alpha\rangle|^{2}}{\lambda-2 \mu}+\frac{|\langle\bar{u}(i \lambda), j \alpha\rangle|^{2}}{\lambda+2 \mu}\right) \\
& -i \pi \theta(2 \mu)|\langle u(2 i \mu), j \alpha\rangle|^{2} .
\end{aligned}
$$

Выражение под знаком интеграла вещественно, поэтому

$$
\operatorname{Im}\langle R(2 i \mu+0) \alpha, j \alpha\rangle=-\pi \theta(2 \mu)|\langle u(2 i \mu), j \alpha\rangle|^{2}<0,
$$

так как $\theta(2 \mu)>0$ и

$$
\begin{aligned}
\langle u(2 i \mu), j \alpha\rangle & =\left\langle u(2 i \mu), j P^{\mathrm{c}} \mathscr{N}_{2}[u, u]\right\rangle=\left\langle u(2 i \mu), j \mathscr{N}_{2}[u, u]\right\rangle \\
& =-\int u_{1}(2 i \mu)(x) N_{2}[u, u](x) d x \\
& =-\frac{1}{2} \int \varphi_{4 \lambda_{1}}(x) F^{\prime \prime}(s(x)) \varphi_{\lambda_{1}}^{2}(x) d x \neq 0
\end{aligned}
$$

в силу условия $\mathbf{F}$. В результате из (12.17) и (12.18) следует, что

$$
\operatorname{Re} Z_{21}^{\prime}<0 \text {. }
$$

Теперь применим метод нормальных форм Пуанкаре к уравнению (12.13). 
Лемма 12.2. Существует замена переменных

$$
z_{1}=z+c_{20} z^{2}+c_{11} z \bar{z}+c_{02} \bar{z}^{2}+c_{30} z^{3}+c_{12} z \bar{z}^{2}+c_{03} \bar{z}^{3}
$$

такая, что новая функиия $z_{1}(t)$ удовлетворяет уравнению

$$
\dot{z}_{1}=i \mu z_{1}+i K\left|z_{1}\right|^{2} z_{1}+\widehat{Z}_{R}
$$

где

$$
\operatorname{Re}(i K)=\operatorname{Re} Z_{21}^{\prime}<0 .
$$

Для остаточного члена $\widehat{Z}_{R}$ справедлива точно такая же оченка, как и для $\widetilde{Z}_{R}$ :

$$
\left|\widehat{Z}_{R}\right|=\mathscr{R}_{1}\left(|z|+\|f\|_{L^{\infty}}\right)\left[\left(|z|^{2}+\|f\|_{E_{-\beta}}\right)^{2}+|z|\|g\|_{E_{-\beta}}+|z|\|h\|_{E_{-\beta}}\right] .
$$

ДокАЗАТЕЛЬство. Подставим выражение (12.20) для функции $z_{1}$ в уравнение (12.13):

$$
\begin{aligned}
\dot{z}_{1}=(1+ & \left.2 c_{20} z+c_{11} \bar{z}+3 c_{30} z^{2}+c_{12} \bar{z}^{2}\right) \dot{z}+\left(c_{11} z+2 c_{02} \bar{z}+2 c_{12} z \bar{z}+3 c_{03} \bar{z}^{2}\right) \dot{\bar{z}} \\
=i \mu z & +Z_{2}\left(z^{2}+2 z \bar{z}+\bar{z}^{2}\right)+Z_{3}\left(z^{3}+3 z^{2} \bar{z}+3 z \bar{z}^{2}+\bar{z}^{3}\right) \\
& +Z_{30}^{\prime} z^{3}+Z_{21}^{\prime} z^{2} \bar{z}+Z_{12}^{\prime} z \bar{z}^{2}+Z_{03}^{\prime} \bar{z}^{3}+\widetilde{Z}_{R} \\
& +\left(2 c_{20} z+c_{11} \bar{z}\right)\left(i \mu z+Z_{2}\left(z^{2}+2 z \bar{z}+\bar{z}^{2}\right)+\mathscr{O}\left(|z|^{3}\right)+\widetilde{Z}_{R}\right) \\
& +\left(3 c_{30} z^{2}+c_{12} \bar{z}^{2}\right)\left(i \mu z+\mathscr{O}\left(|z|^{2}\right)+\widetilde{Z}_{R}\right) \\
& +\left(c_{11} z+2 c_{02} \bar{z}\right)\left(-i \mu \bar{z}+\bar{Z}_{2}\left(\bar{z}^{2}+2 z \bar{z}+z^{2}\right)+\mathscr{O}\left(|z|^{3}\right)+\widetilde{Z}_{R}\right) \\
& +\left(2 c_{12} z \bar{z}+3 c_{03} \bar{z}^{2}\right)\left(-i \mu \bar{z}+\mathscr{O}\left(|z|^{2}\right)+\widetilde{\bar{Z}}_{R}\right) .
\end{aligned}
$$

С другой стороны, подставляя равенство (12.20) в уравнение $(12.21)$, получим $\dot{z}_{1}=i \mu\left(z+c_{20} z^{2}+c_{11} z \bar{z}+c_{02} \bar{z}^{2}+c_{30} z^{3}+c_{12} z \bar{z}^{2}+c_{03} \bar{z}^{3}\right)+i K z^{2} \bar{z}+\mathscr{O}\left(|z|^{4}\right)+\widehat{Z}_{R}$.

Сравнивая коэффициенты при $z^{2}, z \bar{z}$ и $\bar{z}^{2}$ в правых частях равенств (12.24) и (12.25), мы, в частности, находим:

$$
c_{20}=\frac{i Z_{2}}{\mu}, \quad c_{11}=-\frac{2 i Z_{2}}{\mu}, \quad c_{02}=-\frac{3 i Z_{2}}{\mu} .
$$

Далее, сравнивая коэффициенты при $z^{2} \bar{z}$, получим

$$
i K=3 Z_{3}+Z_{21}^{\prime}+\left(4 c_{20}-c_{11}-2 c_{20}\right) Z_{2} .
$$

Так как коэффициенты $Z_{2}$ и $Z_{3}$, определенные в (11.12), чисто мнимые, то из последнего равенства следует неравенство (12.22). Оценка (12.23) для остаточного члена $\widehat{Z}_{R}$ легко проверяется. Лемма доказана.

Умножив (12.21) на $\bar{z}_{1}$ и взяв вещественную часть, получим уравнение для $y=\left|z_{1}\right|^{2}$ :

$$
\dot{y}=2 \operatorname{Re}(i K) y^{2}+Y_{R},
$$

где

$$
\left|Y_{R}\right|=\mathscr{R}_{1}\left(|z|+\left\|f_{1}\right\|_{L^{\infty}}\right)|z|\left[\left(|z|^{2}+\|f\|_{E_{-\beta}}\right)^{2}+|z|\|g\|_{E_{-\beta}}+|z|\|h\|_{E_{-\beta}}\right] .
$$




\section{3. Мажоранты}

В этом разделе мы определим мажоранты и получим для них равномерные оценки.

13.1. Начальные условия и оценка для $g$. Предварительно сформулируем наши предположения о малости начальных данных для функций $z, f$ и $h$. Согласно условию (9.1) мы можем считать, что начальные условия удовлетворяют следующим неравенствам:

$$
\begin{aligned}
|z(0)| & \leqslant \varepsilon^{1 / 2}, \\
\|f(0)\|_{E_{\beta}}=\|h(0)\|_{E_{\beta}} & \leqslant \varepsilon^{3 / 2} h_{0}, \\
\|f(0)\|_{E_{\beta} \cap W} & \leqslant \varepsilon^{1 / 2} f_{0},
\end{aligned}
$$

где $h_{0}$ и $f_{0}$ - некоторые фиксированные постоянные и $\varepsilon>0$ - малое число. Из уравнения (12.20) следует, что $\left|z_{1}\right|^{2} \leqslant|z|^{2}+\mathscr{R}(|z|)|z|^{3}$. Поэтому

$$
y_{0}=y(0)=\left|z_{1}(0)\right|^{2} \leqslant \varepsilon+C(|z(0)|) \varepsilon^{3 / 2} .
$$

Получим также оценку для $g(t)=-e^{A t} k(0)$, где $k(0)=a_{20} z^{2}(0)+a_{11} z(0) \bar{z}(0)+$ $a_{02} \bar{z}^{2}(0)$ и коэффициенты $a_{i j} \in E_{-\beta}$ определены в $(12.9),(12.10)$. Так как коэффициенты $a_{i j}$ принадлежат также $E^{\text {c }}$, то из (10.6) и (13.1) следует, что

$$
\|g(t)\|_{E_{-\beta}} \leqslant \frac{C|z(0)|^{2}}{(1+t)^{3 / 2}} \leqslant \frac{C \varepsilon}{(1+t)^{3 / 2}}, \quad \beta>\frac{5}{2} .
$$

13.2. Система мажорант. Определим следующие функции от $T \geqslant 0$ :

$$
\begin{aligned}
& \mathscr{M}_{1}(T)=\max _{0 \leqslant t \leqslant T}|z(t)|\left(\frac{\varepsilon}{1+\varepsilon t}\right)^{-1 / 2}, \\
& \mathscr{M}_{2}(T)=\max _{0 \leqslant t \leqslant T}\left\|f_{1}(t)\right\|_{L^{\infty}}\left(\frac{\varepsilon}{1+\varepsilon t}\right)^{-1 / 2} \log ^{-1}(2+\varepsilon t), \\
& \mathscr{M}_{3}(T)=\max _{0 \leqslant t \leqslant T}\|h(t)\|_{E_{-\beta}}\left(\frac{\varepsilon}{1+\varepsilon t}\right)^{-3 / 2} \log ^{-1}(2+\varepsilon t) .
\end{aligned}
$$

Обозначим $\mathscr{M}=\left(\mathscr{M}_{1}, \mathscr{M}_{2}, \mathscr{M}_{3}\right)$. Основная цель этого раздела - доказать равномерную ограниченность $\mathscr{M}(T)$ для достаточно малых $\varepsilon>0$.

13.3. Оценки остаточных членов. Сначала оценим остаточные члены через соответствующие мажоранты.

I. Оценим остаточный член $Y_{R}$, определенный в (12.29). Из равенства $f=$ $k+g+h$ и оценки (12.29) следует, что

$$
\begin{aligned}
\left|Y_{R}\right|= & \mathscr{R}_{2}\left(|z|+\left\|f_{1}\right\|_{L^{\infty}}\right)|z|\left[\left(|z|^{2}+\|g\|_{E_{-\beta}}+\|h\|_{E_{-\beta}}\right)^{2}+|z|\left(\|g\|_{E_{-\beta}}+\|h\|_{E_{-\beta}}\right)\right] \\
= & \mathscr{R}(\mathscr{M})\left(\frac{\varepsilon}{1+\varepsilon t}\right)^{1 / 2} \mathscr{M}_{1}\left[\left(\frac{\varepsilon}{1+\varepsilon t} \mathscr{M}_{1}^{2}+\frac{\varepsilon}{(1+t)^{3 / 2}}\right.\right. \\
& \left.+\left(\frac{\varepsilon}{1+\varepsilon t}\right)^{3 / 2} \log (2+\varepsilon t) \mathscr{M}_{3}\right)^{2}
\end{aligned}
$$




$$
\begin{aligned}
& \left.+\left(\frac{\varepsilon}{1+\varepsilon t}\right)^{1 / 2} \mathscr{M}_{1}\left(\frac{\varepsilon}{(1+t)^{3 / 2}}+\left(\frac{\varepsilon}{1+\varepsilon t}\right)^{3 / 2} \log (2+\varepsilon t) \mathscr{M}_{3}\right)\right] \\
= & \mathscr{R}(\mathscr{M}) \frac{\varepsilon^{5 / 2}}{(1+\varepsilon t)^{2} \sqrt{\varepsilon+\varepsilon t}} \log (2+\varepsilon t)(1+|\mathscr{M}|)^{5} .
\end{aligned}
$$

Поэтому

$$
\left|Y_{R}\right|=\mathscr{R}(\mathscr{M}) \frac{\varepsilon^{5 / 2}}{(1+\varepsilon t)^{2} \sqrt{\varepsilon t}} \log (2+\varepsilon t)(1+|\mathscr{M}|)^{5} .
$$

II. Теперь оценим остаточный член $F_{R}$ из уравнения (11.14). Из (12.2) и (11.16) следует, что

$$
\begin{aligned}
\left\|F_{R}\right\|_{E_{\beta} \cap W}= & \mathscr{R}\left(|z|+\left\|f_{1}\right\|_{L^{\infty}}\right)\left[|z|^{3}+\left(|z|+\left\|f_{1}\right\|_{L^{\infty}}\right)\left(|z|^{2}+\|g\|_{E_{-\beta}}+\|h\|_{E_{-\beta}}\right)\right] \\
= & \mathscr{R}(\mathscr{M})\left(\frac{\varepsilon}{1+\varepsilon t}\right)^{3 / 2}\left[\mathscr{M}_{1}^{3}+\left(\mathscr{M}_{1}+\log (2+\varepsilon t) \mathscr{M}_{2}\right)\right. \\
& \left.\times\left(\mathscr{M}_{1}^{2}+\frac{1}{(1+t)^{1 / 2}}+\left(\frac{\varepsilon}{1+\varepsilon t}\right)^{1 / 2} \log (2+\varepsilon t) \mathscr{M}_{3}\right)\right], \quad \beta>\frac{5}{2} .
\end{aligned}
$$

Следовательно, остаточный член $F_{R}$ удовлетворяет оценке

$\left\|F_{R}\right\|_{E_{\beta} \cap W}=\mathscr{R}(\mathscr{M})\left(\frac{\varepsilon}{1+\varepsilon t}\right)^{3 / 2} \log (2+\varepsilon t)\left(\left(\mathscr{M}_{1}^{2}+1\right)\left(\mathscr{M}_{1}+\mathscr{M}_{2}\right)+\varepsilon^{1 / 2}(1+|\mathscr{M}|)^{2}\right)$.

III. Далее оценим остаточный член $\widetilde{F}_{R}=P^{c} \mathscr{N}_{2}[w, w]+F_{R}$. Из (11.10) следует, что

$$
\left\|P^{\mathrm{c}} \mathscr{N}_{2}[w, w]\right\|_{E_{\beta} \cap W}=\mathscr{R}(\mathscr{M}) \frac{\varepsilon}{1+\varepsilon t} \mathscr{M}_{1}^{2} .
$$

Вместе с оценкой (13.10) это означает, что

$$
\left\|\widetilde{F}_{R}\right\|_{E_{\beta} \cap W}=\mathscr{R}(\mathscr{M}) \frac{\varepsilon}{1+\varepsilon t}\left(\mathscr{M}_{1}^{2}+\varepsilon^{1 / 2}(1+|\mathscr{M}|)^{3}\right) .
$$

IV. Наконец, оценим остаточный член $H_{R}=F_{R}+H_{I}$, где $H_{I}$ определено в $(12.11)$ и коэффициенты $C_{n}$ удовлетворяют оценке $(12.12)$. Оценим $C_{n}$ через мажоранты. Из (12.12) следует, что

$$
\begin{aligned}
\left\|C_{n}\right\|_{E_{\beta}}= & \mathscr{R}\left(|z|+\|f\|_{E_{-\beta}}\right)|z|\left(|z|+\|g\|_{E_{-\beta}}+\|h\|_{E_{-\beta}}\right)^{2} \\
= & \mathscr{R}(\mathscr{M})\left(\frac{\varepsilon}{1+\varepsilon t}\right)^{1 / 2} \mathscr{M}_{1}\left[\left(\frac{\varepsilon}{1+\varepsilon t}\right)^{1 / 2} \mathscr{M}_{1}+\frac{\varepsilon}{(1+t)^{3 / 2}}\right. \\
& \left.+\left(\frac{\varepsilon}{1+\varepsilon t}\right)^{3 / 2} \log (2+\varepsilon t) \cdot \mathscr{M}_{2}\right]^{2} .
\end{aligned}
$$

Поэтому

$$
\left\|C_{n}\right\|_{E_{\beta}}=\mathscr{R}(\mathscr{M})\left(\frac{\varepsilon}{1+\varepsilon t}\right)^{3 / 2}\left(\mathscr{M}_{1}^{3}+\varepsilon^{1 / 2}(1+|\mathscr{M}|)^{3}\right), \quad n=0, \pm 1 .
$$


13.4. Оценки для мажорант. Оценим решения динамических уравнений при помощи мажорант и, таким образом, получим соотношения между самими мажорантами.

I. Сначала получим оценку для решения $y(t)$ уравнения $(12.28)$, которое является уравнением Риккати. Аналогично предложению 5.6 работы [7] доказывается, что решение этого уравнения с начальной функцией, удовлетворяющей неравенству (13.4), и остаточным членом, удовлетворяющим оценке (13.9), оценивается как

$$
\left|y-\frac{y_{0}}{1+2 y_{0} \operatorname{Im} K t}\right| \leqslant \mathscr{R}(\mathscr{M}) \frac{\varepsilon^{5 / 2}}{(1+\varepsilon t)^{2} \sqrt{\varepsilon t}} \log (2+\varepsilon t)(1+|\mathscr{M}|)^{5} .
$$

Далее, из оценок (13.4) и (13.13) следует, что

$$
y \leqslant \mathscr{R}(\mathscr{M})\left[\frac{\varepsilon}{1+\varepsilon t}+\left(\frac{\varepsilon}{1+\varepsilon t}\right)^{3 / 2} \log (2+\varepsilon t)(1+|\mathscr{M}|)^{5}\right] .
$$

Так как $|z|^{2} \leqslant y+\mathscr{R}(|z|)|z|^{3}$, то

$$
|z|^{2} \leqslant \mathscr{R}(\mathscr{M})\left[\frac{\varepsilon}{1+\varepsilon t}+\left(\frac{\varepsilon}{1+\varepsilon t}\right)^{3 / 2} \log (2+\varepsilon t)(1+|\mathscr{M}|)^{5}+\left(\frac{\varepsilon}{1+\varepsilon t}\right)^{3 / 2} \mathscr{M}_{1}^{3}\right] .
$$

Принимая во внимание определение (13.6) первой мажоранты $\mathscr{M}_{1}$, получим

$$
\mathscr{M}_{1}^{2}=\mathscr{R}(\mathscr{M})\left(1+\varepsilon^{1 / 2}(1+|\mathscr{M}|)^{5}\right) .
$$

II. Далее рассмотрим уравнение (11.14) для $f$. Решение этого уравнения может быть представлено в виде

$$
f(t)=e^{A t} f(0)+\int_{0}^{t} e^{A(t-\tau)} \widetilde{F}_{R}(\tau) d \tau .
$$

Используя оценки (10.7), (13.3) и (13.11), получим

$$
\begin{aligned}
\left\|f_{1}\right\|_{L^{\infty}} & \leqslant \frac{C}{(1+t)^{1 / 2}}\|f(0)\|_{E_{\beta} \cap W}+\int_{0}^{t} \frac{C}{(1+(t-\tau))^{1 / 2}}\left\|\widetilde{F}_{R}(\tau)\right\|_{E_{\beta} \cap W} d \tau \\
& \leqslant C\left[f_{0}\left(\frac{\varepsilon}{1+t}\right)^{1 / 2}+\mathscr{R}(\mathscr{M})\left(\mathscr{M}_{1}^{2}+\varepsilon^{1 / 2}(1+|\mathscr{M}|)^{3}\right) \int_{0}^{t} \frac{d \tau}{(t-\tau)^{1 / 2}} \frac{\varepsilon}{1+\varepsilon \tau}\right] \\
& \leqslant C\left(\frac{\varepsilon}{1+\varepsilon t}\right)^{1 / 2} \log (2+\varepsilon t)\left[f_{0}+\mathscr{R}(\mathscr{M})\left(\mathscr{M}_{1}^{2}+\varepsilon^{1 / 2}(1+|\mathscr{M}|)^{3}\right)\right]
\end{aligned}
$$

Отсюда и из определения (13.7) второй мажоранты $\mathscr{M}_{2}$ следует, что

$$
\mathscr{M}_{2}=\mathscr{R}(\mathscr{M})\left(\mathscr{M}_{1}^{2}+\varepsilon^{1 / 2}(1+|\mathscr{M}|)^{3}\right) .
$$

III. Наконец, рассмотрим уравнение $(12.5)$ для $h$. Решение $h(t)$ этого уравнения выражается формулой

$$
h(t)=e^{A t} h(0)+\int_{0}^{t} e^{A(t-\tau)} H_{R}(\tau) d \tau .
$$


Используя оценки (10.6), (10.8), (13.2), (13.10) и (13.12), получим

$$
\begin{aligned}
\|h\|_{E_{-\beta}} \leqslant & \frac{C}{(1+t)^{3 / 2}}\|h(0)\|_{E_{\beta}}+\int_{0}^{t} \frac{C}{(1+(t-\tau))^{3 / 2}} \\
& \times\left(\left\|F_{R}(\tau)\right\|_{E_{\beta}}+\sum_{m}\left\|C_{m}(\tau)\right\|_{E_{\beta}}\right) d \tau \\
\leqslant C & {\left[h_{0}\left(\frac{\varepsilon}{1+t}\right)^{3 / 2}+\mathscr{R}(\mathscr{M})\left(\left(\mathscr{M}_{1}^{2}+1\right)\left(\mathscr{M}_{1}+\mathscr{M}_{2}\right)+\varepsilon^{1 / 2}(1+|\mathscr{M}|)^{2}\right)\right.} \\
& \times \int_{0}^{t} \frac{\log (2+\varepsilon \tau) d \tau}{(1+(t-\tau))^{3 / 2}}\left(\frac{\varepsilon}{1+\varepsilon \tau}\right)^{3 / 2} \\
& \left.+\sum_{m} \mathscr{R}(\mathscr{M})\left(\mathscr{M}_{1}^{3}+\varepsilon^{1 / 2}(1+|\mathscr{M}|)^{3}\right) \int_{0}^{t} \frac{d \tau}{(1+(t-\tau))^{3 / 2}}\left(\frac{\varepsilon}{1+\varepsilon \tau}\right)^{3 / 2}\right] .
\end{aligned}
$$

Следовательно,

$$
\begin{aligned}
\|h\|_{E_{-\beta}} \leqslant C & \left(\frac{\varepsilon}{1+\varepsilon t}\right)^{3 / 2} \log (2+\varepsilon t)\left[h_{0}+\mathscr{R}(\mathscr{M})\left(\left(\mathscr{M}_{1}^{2}+1\right)\left(\mathscr{M}_{1}+\mathscr{M}_{2}\right)\right.\right. \\
& \left.\left.+\varepsilon^{1 / 2}(1+|\mathscr{M}|)^{3}\right)\right] .
\end{aligned}
$$

В результате, используя определение (13.8), получим

$$
\mathscr{M}_{3}=\mathscr{R}(\mathscr{M})\left[\left(\mathscr{M}_{1}^{2}+1\right)\left(\mathscr{M}_{1}+\mathscr{M}_{2}\right)+\varepsilon^{1 / 2}(1+|\mathscr{M}|)^{3}\right] .
$$

13.5. Равномерные оценки для мажорант. Покажем, что при достаточно малых $\varepsilon$ все мажоранты $\mathscr{M}_{i}$ равномерно ограничены по $T$ и $\varepsilon$.

Комбинируя оценки (13.14), (13.15) и (13.17) для мажорант, получим следующее неравенство:

$$
\mathscr{M}^{2}=\mathscr{R}(\mathscr{M})\left[1+\left(\mathscr{M}_{1}^{4}+1\right)\left(\mathscr{M}_{1}^{2}+\mathscr{M}_{2}^{2}\right)+\varepsilon^{1 / 2}(1+|\mathscr{M}|)^{6}\right] .
$$

Заменяя $\mathscr{M}_{1}^{2}$ и $\mathscr{M}_{2}$ в правой части их оценками $(13.14)$ и (13.15), получим

$$
\mathscr{M}^{2}=\mathscr{R}(\mathscr{M})\left(1+\varepsilon^{1 / 2} F(\mathscr{M})\right)
$$

с некоторой непрерывной функцией $F(\mathscr{M})$. Отсюда следует равномерная ограниченность функции $\mathscr{M}(T)$, так как величина $\mathscr{M}(0)$ мала и функция $\mathscr{M}(T)$ непрерывна. В результате мы доказали, что для достаточно малых $\varepsilon$ существует постоянная величина $M$, не зависящая от $T$ и $\varepsilon$, такая, что $|\mathscr{M}(T)| \leqslant M$. Из этого неравенства и определений (13.6)-(13.8) мажорант $\mathscr{M}_{i}$ немедленно вытекают следующие оценки:

$$
\begin{aligned}
|z(t)| \leqslant M\left(\frac{\varepsilon}{1+\varepsilon t}\right)^{1 / 2}, & \left\|f_{1}(t)\right\|_{L^{\infty}} \leqslant M\left(\frac{\varepsilon}{1+\varepsilon t}\right)^{1 / 2} \log (1+\varepsilon t), \\
\|f(t)\|_{E_{-\beta}} \leqslant M\left(\frac{\varepsilon}{1+\varepsilon t}\right), & \|h(t)\|_{E_{-\beta}} \leqslant M\left(\frac{\varepsilon}{1+\varepsilon t}\right)^{3 / 2} \log (1+\varepsilon t),
\end{aligned}
$$

где $\beta>5 / 2$. Таким образом, мы доказали следующий результат. 
Теорема 13.1. Пусть выполнены условия теоремы 9.1. Тогда для достаточно малых $\varepsilon>0$ существуют функции $z(t) \in C^{1}(\mathbb{R})$ u $f(x, t) \in C(\mathbb{R}, E)$, а также константа $M>0$, такие, что при всех $t \geqslant 0$ решение уравнения (2.3) можно записать в виде

$$
Y(x, t)=S(x)+(z(t)+\bar{z}(t)) u+f(x, t) .
$$

Кроме того, для функиий z(t) и $f(x, t)$ справедливы оценки

$$
|z(t)| \leqslant M\left(\frac{\varepsilon}{1+\varepsilon t}\right)^{1 / 2}, \quad\|f(t)\|_{E_{-\beta}} \leqslant M\left(\frac{\varepsilon}{1+\varepsilon t}\right), \quad \beta>\frac{5}{2}, \quad t \geqslant 0 .
$$

\section{4. Долговременные асимптотики}

14.1. Долговременное поведение $z(t)$. Так как оценка $(12.23)$ для $\widehat{Z}_{R}$ отличается от оценки для $Y_{R}$ только множителем $|z|$, то из (13.9) и ограниченности мажорант следует, что

$$
\left|\widehat{Z}_{R}(t)\right| \leqslant C \varepsilon^{2}(1+\varepsilon t)^{-3 / 2}(\varepsilon t)^{-1 / 2} \log (2+\varepsilon t) .
$$

С другой стороны, учитывая, что $\left|z_{1}\right|^{2}=y$, из неравенства (13.13) получим

$$
\left.|| z_{1}(t)\right|^{2}-\frac{y_{0}}{1+2 \operatorname{Im} K y_{0} t} \mid \leqslant \frac{C \varepsilon^{5 / 2} \log (2+\varepsilon t)}{(1+\varepsilon t)^{2} \sqrt{\varepsilon t}} .
$$

Поэтому уравнение (12.21) можно записать в виде

$$
\dot{z}_{1}=i \mu z_{1}+i K \frac{y_{0}}{1+2 \operatorname{Im} K y_{0} t} z_{1}+Z_{1},
$$

где остаточный член $Z_{1}$ в силу (13.18) и (14.1) удовлетворяет оценке

$$
\left|Z_{1}(t)\right| \leqslant C \varepsilon^{2}(1+\varepsilon t)^{-3 / 2}(\varepsilon t)^{-1 / 2} \log (2+\varepsilon t) .
$$

Так как $y_{0}=\varepsilon+\mathscr{O}\left(\varepsilon^{3 / 2}\right)$ в силу (13.4), то мы можем заменить в уравнении $(14.2)$ константу $2 \operatorname{Im} K y_{0}$ константой $k \varepsilon$. Обозначим далее $\rho=\operatorname{Re} K / \operatorname{Im} K$. Тогда решение $z_{1}$ уравнения (14.2) может быть записано в следующем виде:

$$
\begin{aligned}
z_{1}(t) & =\frac{e^{i \mu t}}{(1+k \varepsilon t)^{1 / 2-i \rho}}\left[z_{1}(0)+\int_{0}^{t} e^{-i \mu s}(1+k \varepsilon s)^{1 / 2-i \rho} Z_{1}(s) d s\right] \\
& =z_{\infty} \frac{e^{i \mu t}}{(1+k \varepsilon t)^{1 / 2-i \rho}}+z_{R}(t),
\end{aligned}
$$

где

$$
\begin{aligned}
z_{\infty} & =z_{1}(0)+\int_{0}^{\infty} e^{-i \mu s}(1+k \varepsilon s)^{1 / 2-i \rho} Z_{1}(s) d s \\
z_{R}(t) & =-\int_{t}^{\infty} e^{i \mu t}\left(\frac{1+k \varepsilon s}{1+k \varepsilon t}\right)^{1 / 2-i \rho} Z_{1}(s) d s .
\end{aligned}
$$


Из оценки (14.3) остаточного члена $Z_{1}$ следует, что $\left|z_{R}(t)\right| \leqslant C \varepsilon(1+\varepsilon t)^{-1} \log (2+$ $\varepsilon t)$. Таким образом, для функции $z_{1}(t)$ справедлива следующая асимптотика:

$$
z_{1}(t)=z_{\infty} \frac{e^{i \mu t}}{(1+k \varepsilon t)^{1 / 2-i \rho}}+\mathscr{O}\left(t^{-1} \log t\right), \quad t \rightarrow+\infty .
$$

Так как $z=z_{1}+\mathscr{O}\left(t^{-1}\right)$ в силу (12.20) и (13.18), то из (14.4) немедленно следует асимптотика для $z(t)$ :

$$
z(t)=z_{\infty} \frac{e^{i \mu t}}{(1+k \varepsilon t)^{1 / 2-i \rho}}+\mathscr{O}\left(t^{-1} \log t\right), \quad t \rightarrow+\infty .
$$

14.2. Солитонная асимптотика. Докажем главную теорему 9.1. Согласно теореме 13.1 , решение $Y(x, y)$ уравнения $(2.3)$ записывается в виде

$$
Y(t)=S+w(t)+f(t), \quad \text { где } w(t)=z(t) u+\bar{z}(t) \bar{u},
$$

и для функций $z(t)$ и $f(t)$ справедливы оценки (13.20). Мы можем включить функцию $w(t)$ в остаточный член $r_{+}(t)$ асимптотики $(9.2)$, так как $z(t) \sim t^{-1 / 2}$ ввиду (14.5). Поэтому для доказательства этой асимптотики нам достаточно выделить дисперсионную волну $W_{0}(t) \Phi_{+}$из функции $f(t)$. Перепишем уравнение (11.14) в виде

$$
\dot{f}=A_{0} f+\mathscr{V} f+P^{\mathrm{c}} \mathscr{N}
$$

где

$$
A_{0}=\left(\begin{array}{cc}
0 & 1 \\
-\Delta+m^{2} & 0
\end{array}\right), \quad \mathscr{V}=\left(\begin{array}{cc}
0 & 0 \\
V & 0
\end{array}\right)
$$

и $\mathscr{N}$ определено в (10.3). Для решения $f(t)$ уравнения (14.7) напишем интегральное представление:

$$
\begin{aligned}
f(t) & =W_{0}(t) f(0)+\int_{0}^{t} W_{0}(t-\tau) Q(\tau) d \tau \\
& =W_{0}(t)\left(f(0)+\int_{0}^{\infty} W_{0}(-\tau) Q(\tau) d \tau\right)-\int_{t}^{\infty} W_{0}(t-\tau) Q(\tau) d \tau \\
& =W_{0}(t) \phi_{+}+r_{+}(t)
\end{aligned}
$$

где $Q(t)$ - векторная функция с координатами

$$
Q_{1}=\left(P^{\mathrm{c}} \mathscr{N}\right)_{1}=-\left(P^{\mathrm{d}} \mathscr{N}\right)_{1}, \quad Q_{2}=\left(P^{\mathrm{c}} \mathscr{N}\right)_{2}-V f_{1}=\left(P^{\mathrm{c}} \mathscr{N}_{2}[w, w]\right)_{2}+\left(F_{R}\right)_{2}-V f_{1} .
$$

Для получения асимптотики (9.2) достаточно проверить, что все интегралы в (14.8) сходятся в норме пространства $E$ и, кроме того,

$$
\left\|r_{+}(t)\right\|_{E}=\mathscr{O}\left(t^{-1 / 3}\right), \quad t \rightarrow \infty .
$$

Так как функция $\varphi_{\lambda_{1}}$ вещественна, то из определения (10.5) и равенства (11.13) следует, что

$$
Q_{1}=-\left(P^{\mathrm{d}} \mathscr{N}\right)_{1}=\frac{i}{\delta} \varphi_{\lambda_{1}}(\langle\mathscr{N}, j u\rangle-\langle\mathscr{N}, j \bar{u}\rangle)=0
$$


Рассмотрим функцию $Q_{2}(t)$. Из равенств $(10.5),(11.10)$ и $(11.12)$ получим $\left(P^{\mathrm{c}} \mathscr{N}_{2}[w, w]\right)_{2}=N_{2}[w, w]-\left(P^{\mathrm{d}} \mathscr{N}_{2}[w, w]\right)_{2}=\left(z^{2}+2 z \bar{z}+\bar{z}^{2}\right)\left(N_{2}[u, u]-2 i \mu u_{1} Z_{2}\right)$.

Поэтому, принимая во внимание равенства (12.2), (12.3), представим функцию $Q_{2}(t)$ следующим образом:

$$
Q_{2}=q_{20} z^{2}+2 q_{11} z \bar{z}+q_{02} \bar{z}^{2}+Q_{R},
$$

где $q_{i j}=N_{2}\left[u_{1}, u_{1}\right]-2 i Z_{2} \mu u_{1}-V\left(a_{i j}\right)_{1}, Q_{R}=\left(F_{R}\right)_{2}-V\left(f_{1}-k_{1}\right)$, а $\left(a_{i j}\right)_{1}$ и $k_{1}$ - первые компоненты векторных функций $a_{i j}$ и $k$. Согласно оценкам (11.15) и $(13.18)$,

$$
\left\|\left(F_{R}\right)_{2}\right\|_{L^{2}}=\mathscr{O}\left(t^{-3 / 2} \log t\right), \quad t \rightarrow \infty .
$$

Из равенства (12.2) и оценок (13.5) и (13.18) следует, что

$$
\left\|V\left(f_{1}-k_{1}\right)\right\|_{L^{2}}=\left\|V\left(g_{1}+h_{1}\right)\right\|_{L^{2}}=\mathscr{O}\left(t^{-3 / 2} \log t\right), \quad t \rightarrow \infty .
$$

Поэтому остаточный член $Q_{R}$ в $(14.11)$ имеет асимптотику

$$
\left\|Q_{R}\right\|_{L^{2}}=\mathscr{O}\left(t^{-3 / 2} \log t\right), \quad t \rightarrow \infty .
$$

Следовательно, интегралы в (14.8) с функцией $Q_{R}$ сходятся в пространстве $E$, и вклад этой функции в остаточный член $r_{+}(t)$ имеет порядок $\mathscr{O}\left(t^{-1 / 2} \log t\right)$.

Осталось оценить вклады в $r_{+}(t)$ от квадратичных членов в правой части равенства (14.11). Очевидно, что функции $q_{i j}(x)$ принадлежат $L^{2}$, но функции $z^{2}(t), \bar{z}^{2}(t)$ и $z(t) \bar{z}(t)$ убывают медленно, как $\mathscr{O}\left(t^{-1}\right)$. Поэтому мы не можем получить абсолютную сходимость интегралов. Однако мы можем определить такие интегралы как пределы. Например,

$$
\int_{t}^{\infty} W_{0}(t-\tau)\left(\begin{array}{c}
0 \\
q_{11}(\tau)
\end{array}\right) z \bar{z} d \tau:=\lim _{T \rightarrow \infty} \int_{t}^{T} W_{0}(t-\tau)\left(\begin{array}{c}
0 \\
q_{11}(\tau)
\end{array}\right) z \bar{z} d \tau .
$$

Докажем существование этих пределов в пространстве $E$.

Сначала рассмотрим интеграл (14.13). Из асимптотики (14.5) следует, что $z \bar{z} \sim(1+k \varepsilon t)^{-1}$. Покажем, что вклад интеграла $(14.13)$ в остаточный член $r_{+}(t)$ равен $\mathscr{O}\left(t^{-1}\right)$.

Лемма 14.1. Пусть $q(x) \in L^{2}(\mathbb{R})$. Тогда

$$
I(t):=\left\|\int_{t}^{\infty} W_{0}(-\tau)\left(\begin{array}{l}
0 \\
q
\end{array}\right) \frac{d \tau}{1+\tau}\right\|_{E}=\mathscr{O}\left(t^{-1}\right), \quad t \rightarrow \infty .
$$

ДоказАтельство. Обозначим $\omega=\omega(\xi)=\sqrt{\xi^{2}+m^{2}}$. Тогда

$$
I(t) \sim\left\|\int_{t}^{\infty}\left(\begin{array}{l}
-\sin (\omega \tau) \widehat{q}(\xi) \\
-\cos (\omega \tau) \widehat{q}(\xi)
\end{array}\right) \frac{d \tau}{1+\tau}\right\|_{L^{2} \oplus L^{2}} \leqslant \frac{C}{1+t}\left\|\frac{\widehat{q}(\xi)}{\omega(\xi)}\right\|_{L^{2}} \leqslant \frac{C_{1}}{1+t},
$$

так как из формулы интегрирования по частям следует, что

$$
\begin{aligned}
\left|\int_{t}^{\infty} \frac{e^{i \omega \tau}}{1+\tau} d \tau\right| & =\left|\int_{t}^{\infty} \frac{d e^{i \omega \tau}}{i \omega(1+\tau)} d \tau\right| \leqslant\left|\frac{e^{i \omega \tau}}{\omega(1+t)}\right|+\left|\int_{t}^{\infty} \frac{e^{i \omega \tau}}{\omega(1+\tau)^{2}} d \tau\right| \\
& \leqslant \frac{C}{\omega(1+t)} .
\end{aligned}
$$

Лемма доказана. 
Теперь рассмотрим интегралы (14.13) с функциями $q_{20}(x) z^{2}$ и $q_{02}(x) \bar{z}^{2}$ вместо функции $q_{11}(x) z \bar{z}$ и покажем, что вклад этих интегралов в остаточный член $r_{+}(t)$ равен $\mathscr{O}\left(t^{-1 / 3}\right)$. Из асимптотики (14.5) следует, что

$$
z^{2} \sim \frac{e^{2 i \mu \tau}}{(1+k \varepsilon t)^{1-2 i \rho}}, \quad \bar{z}^{2} \sim \frac{e^{-2 i \mu \tau}}{(1+k \varepsilon t)^{1+2 i \rho}} .
$$

Кроме того, нетрудно проверить, что $q_{02}, q_{20} \in L^{1}(\mathbb{R})$. Поэтому остается доказать следующую лемму.

Лемма 14.2. Пустъ $q(x) \in L^{2}(\mathbb{R}) \cap L^{1}(\mathbb{R})$. Тогда

$$
I_{ \pm}(t):=\left\|\int_{t}^{\infty} W_{0}(-\tau)\left(\begin{array}{l}
0 \\
q
\end{array}\right) \frac{e^{ \pm 2 i \mu \tau} d \tau}{(1+\tau)^{1 \mp 2 i \rho}}\right\|_{E}=\mathscr{O}\left(t^{-1 / 3}\right), \quad t \rightarrow \infty .
$$

ДокАЗАТЕльство. Мы рассмотрим только интеграл $I_{-}(t)$, содержащий экспоненту $e^{-2 i \mu \tau}$. Интеграл с экспонентой $e^{+2 i \mu \tau}$ рассматривается аналогично. Кроме того, для простоты доказательства мы опустим множитель $(1+t)^{2 i \rho}$. Аналогично (14.15) получим

$$
I_{-}(t) \sim\left\|\int_{t}^{\infty}\left(\begin{array}{l}
-\sin (\omega \tau) \widehat{q}(\xi) \\
-\cos (\omega \tau) \widehat{q}(\xi)
\end{array}\right) \frac{e^{-2 i \mu \tau} d \tau}{1+\tau}\right\|_{L^{2} \oplus L^{2}} .
$$

Представив $\sin \omega \tau$ и $\cos \omega \tau$ как линейную комбинацию экспонент $e^{i \omega \tau}$ и $e^{-i \omega \tau}$, получим два интеграла с экспонентами $e^{-i(\omega+2 \mu) \tau}$ и $e^{i(\omega-2 \mu) \tau}$ соответственно. Интеграл, содержащий “нерезонансный” множитель $e^{-i(\omega+2 \mu) \tau}$, оценивается аналогично (14.15)-(14.16), и его вклад в остаточный член $r_{+}(t)$ равен $\mathscr{O}\left(t^{-1}\right)$. Осталось показать, что

$$
J(t)=\left\|\int_{t}^{\infty} \frac{e^{i(\omega-2 \mu) \tau} \widehat{q}(\xi) d \tau}{1+\tau}\right\|_{L^{2}}=\mathscr{O}\left(t^{-1 / 3}\right) .
$$

Для некоторого фиксированного $0<\alpha<1$ определим функцию

$$
\chi_{\tau}(\xi)= \begin{cases}1, & |\omega(\xi)-2 \mu| \leqslant(1+\tau)^{-\alpha} \\ 0, & |\omega(\xi)-2 \mu|>(1+\tau)^{-\alpha}\end{cases}
$$

Тогда

$$
\begin{aligned}
J(t) & \leqslant\left\|\int_{t}^{\infty} \frac{e^{i(2 \omega-\mu) \tau} \chi_{\tau}(\xi) \widehat{q}(\xi) d \tau}{1+\tau}\right\|_{L^{2}}+\left\|\int_{t}^{\infty} \frac{e^{i(2 \omega-\mu) \tau}\left(1-\chi_{\tau}(\xi)\right) \widehat{q}(\xi) d \tau}{1+\tau}\right\|_{L^{2}} \\
& =J_{1}(t)+J_{2}(t) .
\end{aligned}
$$

Так как функция $\widehat{q}(\xi)$ ограничена и $\left\|\chi_{\tau}\right\|_{L^{2}} \leqslant(1+\tau)^{-\alpha / 2}$, то имеем место оценка $J_{1}(t) \leqslant\|\widehat{q}\|_{L^{\infty}}(1+t)^{-\alpha / 2}$. С другой стороны, интегрируя по частям, получим

$$
\begin{aligned}
J_{2}(t) & =\left\|\int_{t}^{\infty} \frac{\left(1-\chi_{\tau}(\xi)\right) \widehat{q}(\xi) d e^{i(2 \omega-\mu) \tau}}{(2 \omega-\mu)(1+\tau)}\right\|_{L^{2}} \\
& \leqslant \frac{C(1+t)^{\alpha}}{1+t}\|\widehat{q}\|_{L^{2}}+C \int_{t}^{\infty} \frac{(1+\tau)^{\alpha} d \tau}{(1+\tau)^{2}}\|\widehat{q}\|_{L^{2}} \leqslant \frac{C\|\widehat{q}\|_{L^{2}}}{(1+t)^{1-\alpha}} .
\end{aligned}
$$

Приравнивая показатели степени: $\alpha / 2=1-\alpha$, получим $\alpha=2 / 3$. Лемма доказана. 


\section{Глава III. Примеры нелинейных потенциалов}

В этой главе мы построим примеры нелинейных потенциалов, удовлетворяющих спектральным условиям первой и второй глав. Сначала рассмотрим кусочно параболические потенциалы, склеенные в двух точках, а затем аппроксимируем их гладкими функциями.

\section{5. Кусочно параболические потенциалы}

Рассмотрим уравнение (1.1)

$$
\ddot{\psi}(x, t)=\psi^{\prime \prime}(x, t)-U_{0}^{\prime}(\psi(x, t)), \quad x \in \mathbb{R},
$$

с кусочно параболическим потенциалом

$$
U_{0}(\psi)= \begin{cases}\left(1-b \psi^{2}\right) / 2, & |\psi| \leqslant \gamma \\ d(\psi \mp 1)^{2} / 2, & \pm \psi \geqslant \gamma\end{cases}
$$

где $b, d>0$ и $0<\gamma<1$ - некоторые константы. Найдем условия на $b$ и $d$, при которых $U_{0}(\psi) \in C^{1}(\mathbb{R})$. Приравнивая значения функции $U_{0}$ и ее производной в точке $\gamma$, получим

$$
b=\frac{1}{\gamma}, \quad d=\frac{1}{1-\gamma}, \quad 0<\gamma<1 .
$$

Заметим, что вторая производная $U_{0}^{\prime \prime}(\psi)$ является кусочно постоянной функцией с разрывами в точках $\psi= \pm \gamma$. Рассмотрим стационарное уравнение:

$$
s_{0}^{\prime \prime}(x)=U_{0}^{\prime}\left(s_{0}(x)\right)= \begin{cases}-b s_{0}(x), & 0<s_{0}(x) \leqslant \gamma \\ d\left(s_{0}(x)-1\right), & s_{0}(x)>\gamma\end{cases}
$$

Найдем нечетное ненулевое решение (кинк) этого уравнения:

$$
s_{0}(x)= \begin{cases}C \sin \sqrt{b} x, & 0<x \leqslant q, \\ A e^{-\sqrt{d} x}+1, & x>q,\end{cases}
$$

где $C>\gamma, A<0, q=(1 / \sqrt{b}) \arcsin (\gamma / C)$. Приравнивая значения функции $s_{0}(x)$ и ее производной в точке $x=q$ и используя равенства (15.3) для $b$ и $d$, получим

$$
C=\sqrt{\gamma}, \quad A=(\gamma-1) e^{\sqrt{\gamma /(1-\gamma)} \arcsin \sqrt{\gamma}}, \quad q=\sqrt{\gamma} \arcsin \sqrt{\gamma}
$$

15.1. Линеаризованное уравнение. Линеаризуем уравнение (15.1) вблизи кинка $s_{0}(x)$, представив решение $\psi(t)$ этого уравнения в виде суммы $\psi(t)=$ $s_{0}+\phi(t)$. Подставив это разложение в уравнение $(15.1)$, получим

$$
\ddot{\phi}(x, t)=\phi^{\prime \prime}(x, t)-U_{0}^{\prime}\left(s_{0}(x)+\phi(x, t)\right)+U_{0}^{\prime}\left(s_{0}(x)\right) .
$$


Принимая во внимание равенство (15.2), запишем последнее уравнение в виде

$$
\ddot{\phi}(t)=-H_{0} \phi(t)+\mathscr{N}(\phi(t)), \quad t \in \mathbb{R},
$$

где

$$
H_{0}=-\frac{d^{2}}{d x^{2}}+W_{0}(x), \quad W_{0}(x)=U_{0}^{\prime \prime}\left(s_{0}(x)\right)= \begin{cases}-b, & |x| \leqslant q \\ d, & |x|>q\end{cases}
$$

Непрерывный спектр оператора $H_{0}$ совпадает с $[d, \infty)$, а дискретный спектр принадлежит интервалу $[0, d]$. Собственная функция $\varphi(x)$, соответствующая собственному значению $\lambda$, удовлетворяет уравнению

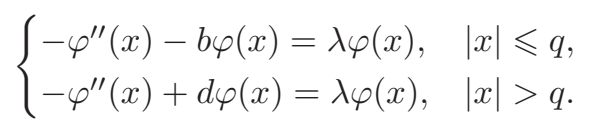

Собственное значение $\lambda_{0}=0$ является основным состоянием. Ему соответствует четная положительная собственная функция $\varphi_{0}(x)=s_{0}^{\prime}(x)$. Следовательно, собственная функция $\varphi_{1}(x)$, соответствующая следующему собственному значению $\lambda_{1}>0$ (если оно существует), должна быть нечетной.

15.2. Нечетные собственные функции. Из уравнения (15.8) следует, что нечетные собственные функции имеют вид

$$
\varphi(x)= \begin{cases}B \sin \beta x, & |x| \leqslant q, \\ A(\operatorname{sgn} x) e^{-\alpha|x|}, & |x|>q,\end{cases}
$$

где $\alpha=\sqrt{d-\lambda}>0$ и $\beta=\sqrt{b+\lambda}>0$. Приравнивая значения функции $\varphi(x)$ и ее производной слева и справа от точки $x=q$, получим уравнения

$$
A e^{-\alpha q}=B \sin \beta q, \quad-A \alpha e^{-\alpha q}=B \beta \cos \beta q,
$$

где

$$
\alpha^{2}+\beta^{2}=b+d
$$

Система (15.10) имеет ненулевое решение только в том случае, если ее определитель равен нулю, т. е.

$$
-\alpha=\beta \cot \beta q \text {. }
$$

Умножим обе части уравнения (15.12) на $q$ и обозначим $\xi=\beta q, \eta=\alpha q$. Принимая во внимание соотношение (15.11), получим следующую систему уравнений:

$$
-\eta=\xi \cot \xi, \quad \xi^{2}+\eta^{2}=R^{2}
$$

где через $R=q \sqrt{b+d}$ мы обозначили радиус окружности на плоскости $(\xi, \eta)$. Подставляя сюда выражения для $b, d$ и $q$ через параметр $\gamma$ из равенств $(15.3)$ и (15.6), получим

$$
R=\frac{q}{\sqrt{\gamma(1-\gamma)}}=\frac{\arcsin \sqrt{\gamma}}{\sqrt{1-\gamma}}
$$




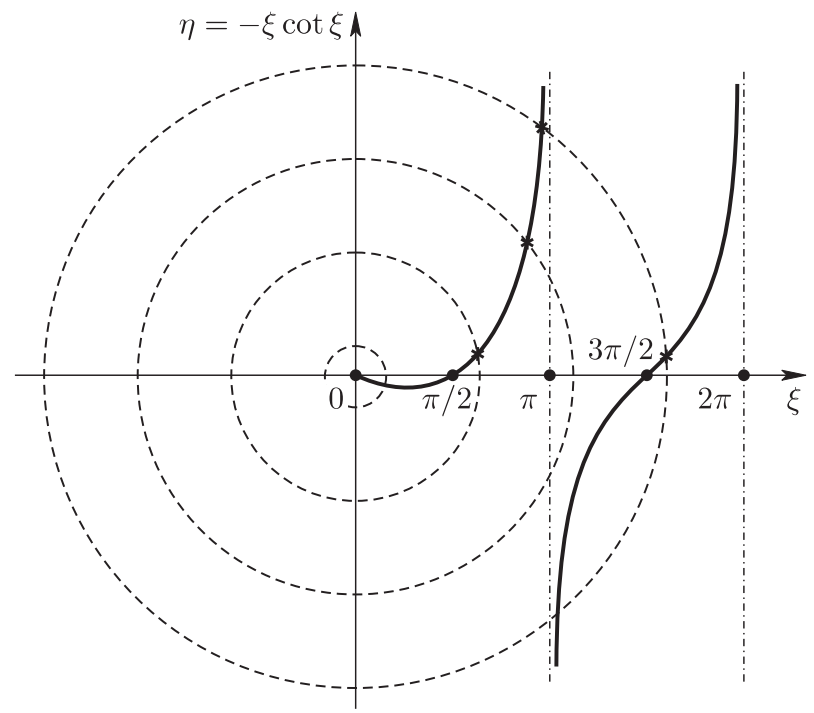

Рис. 5. Графическое решение системы (15.13)

Решение системы (15.13) может быть найдено графически (см. рис. 5). Учитывая, что $\eta>0$, получим следующий результат:

$R \in(0, \pi / 2]: \quad$ система (15.13) не имеет решений,

$R \in(\pi / 2,3 \pi / 2]: \quad$ система (15.13) имеет единственное решение,

$R \in(3 \pi / 2,5 \pi / 2]: \quad$ система (15.13) имеет два решения,

Обозначим через $\gamma_{k}, k \in \mathbb{N}$, решение уравнения

$$
\frac{\arcsin \sqrt{\gamma_{k}}}{\sqrt{1-\gamma_{k}}}=\frac{k \pi}{2}, \quad k \in \mathbb{N}
$$

Решая это уравнение численно, получим

$$
\gamma_{1} \sim 0.64643, \gamma_{2} \sim 0.8579, \gamma_{3} \sim 0.92472, \gamma_{4} \sim 0.95359, \gamma_{5} \sim 0.96856, \ldots
$$

Из (15.15) следует, что

$\gamma \in\left(0, \gamma_{1}\right]: \quad$ нет ненулевых нечетных собственных функций, $\gamma \in\left(\gamma_{1}, \gamma_{3}\right]: \quad$ существует одна линейно независимая нечетная собственная функция,

$\gamma \in\left(\gamma_{3}, \gamma_{5}\right]: \quad$ существуют две линейно независимые нечетные собственные функции, 


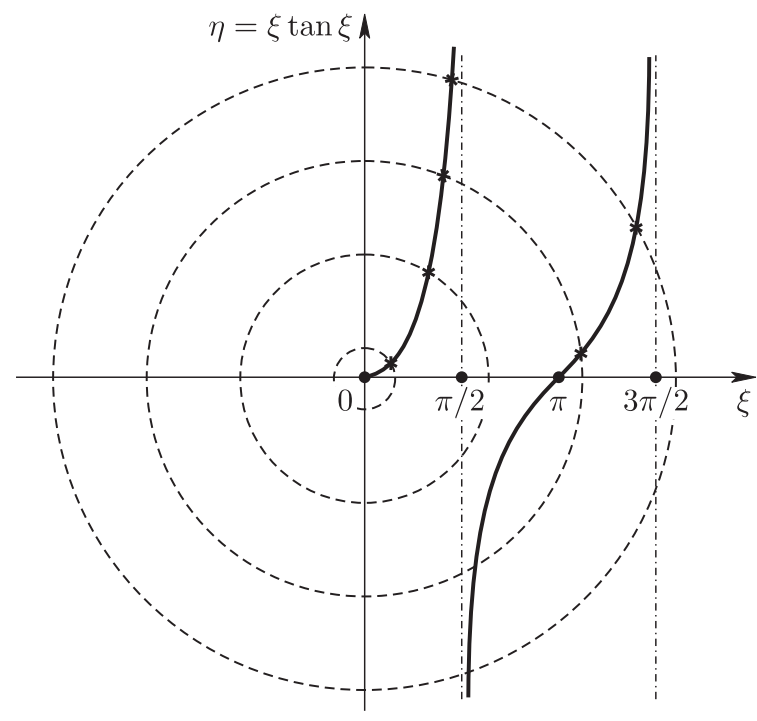

Рис. 6. Графическое решение системы (15.21)

В частности, при $\gamma \in\left(\gamma_{1}, \gamma_{3}\right]$ у нас есть одна нечетная собственная функция и ей соответствует собственное значение

$$
\lambda_{1}=\lambda_{1}(\gamma)=\beta^{2}-b=\frac{\xi^{2}}{q^{2}}-b=\frac{1}{\gamma}\left(\frac{\xi^{2}}{\arcsin ^{2} \sqrt{\gamma}}-1\right)=\frac{1}{\gamma}\left(\frac{\sin ^{2} \xi}{1-\gamma}-1\right)
$$

где $\xi$ - решение уравнения

$$
\frac{\xi^{2}}{\sin ^{2} \xi}=\frac{\arcsin ^{2} \sqrt{\gamma}}{1-\gamma}
$$

15.3. Четные собственные функции. Из уравнения (15.8) следует, что четные собственные функции имеют вид

$$
\varphi(x)= \begin{cases}B \cos \beta x, & |x| \leqslant q \\ A e^{-\alpha|x|}, & |x|>q\end{cases}
$$

Аналогично (15.13) получим следующие уравнения для параметров $\xi=\beta q$ и $\eta=\alpha q$ :

$$
\eta=\xi \tan \xi, \quad \xi^{2}+\eta^{2}=R^{2}
$$

Решение этой системы также найдем графически (см. рис. 6). Получим:

$$
\begin{aligned}
& R \in(0, \pi]: \quad \text { система (15.21) имеет одно решение, } \\
& R \in(\pi, 2 \pi]: \text { система (15.21) имеет два решения, }
\end{aligned}
$$


Заметим, что для любого $\gamma \in(0,1)$ функция $\xi=\arcsin \sqrt{\gamma} \in(0, \pi / 2)$ является решением уравнения (15.21). Это решение соответствует собственному значению $\lambda=0$ и первой четной собственной функции. Итак,

$$
\begin{aligned}
\text { при } \gamma \in\left(0, \gamma_{2}\right]: & \text { существует одна линейно независимая четная } \\
\text { при } \gamma \in\left(\gamma_{2}, \gamma_{4}\right]: & \begin{array}{l}
\text { собственная функция, } \\
\text { собственные функции, }
\end{array}
\end{aligned}
$$

Вывод: при $\gamma \in\left(0, \gamma_{1}\right]$ существует одно собственное значение $\lambda_{0}=0$; при $\gamma \in\left(\gamma_{1}, \gamma_{2}\right]$ существуют два собственных значения $\lambda_{0}=0$ и $0<\lambda_{1}<d$ и т. д. (см. рис. 7).

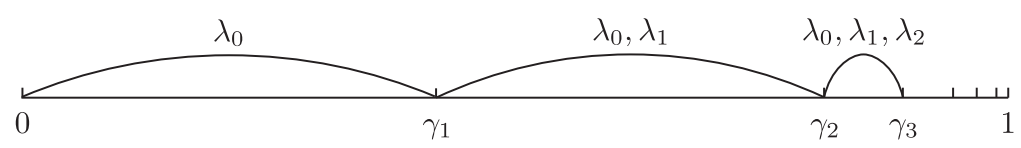

Рис. 7. Дискретный спектр

15.4. Спектральные условия. Очевидно, что для потенциала $U_{0}$ выполнены условия $\mathbf{U} \mathbf{1}$ и $\mathbf{U 2}$, за исключением гладкости в точках $\psi= \pm \gamma$. Из (15.9) и (15.20) следует, что резонанс возможен только при $\alpha=0$ и, следовательно, при $\gamma=\gamma_{k}, k \in \mathbb{N}$. Поэтому условие $\mathbf{U 3}$ выполнено при $\gamma \in(0,1) \backslash\left\{\gamma_{k}\right\}$. Кроме того, при всех $\gamma \in\left(0, \gamma_{1}\right]$ выполнено условие D1. Следовательно, при $\gamma \in\left(0, \gamma_{1}\right)$ выполнены все спектральные условия первой главы, за исключением гладкости потенциала в точках $\pm \gamma$.

Для того чтобы выполнялись условия второй главы, достаточно найти значения $\gamma \in\left(\gamma_{1}, \gamma_{2}\right)$, при которых выполнены условия (1.7) и $\mathbf{F}$, так как мы уже доказали выше, что при таких $\gamma$ оператор $H_{0}$ имеет ровно два собственных значения: $\lambda_{0}=0$ и $\lambda_{1} \in(0, d)$.

Лемма 15.1. Условие (1.7) выполнено при всех $\gamma \in\left(\gamma_{1}, \gamma_{2}\right)$. Условие $\mathbf{F}$ въполнено при всех $\gamma \in\left(\gamma_{1}, \gamma_{2}\right)$, за исключением единственной точки $\gamma_{*}$.

ДокАзАтельство. 1) Согласно (15.18), (15.19), неравенство (1.7) с $m^{2}=d$ эквивалентно неравенству

$$
\frac{4}{\gamma}\left(\frac{\sin ^{2} \xi(\gamma)}{1-\gamma}-1\right)>\frac{1}{1-\gamma},
$$

где $\xi(\gamma) \in(\pi / 2, \pi)$ - решение уравнения (15.19). После простых преобразований получим $4 \cos ^{2} \xi(\gamma)<3 \gamma$. Следовательно, достаточно проверить, что для всех $\gamma \in\left(\gamma_{1}, \gamma_{2}\right)$

$$
\frac{\pi}{2}<\xi(\gamma)<\pi-\arccos \frac{\sqrt{3 \gamma}}{2}
$$

Так как функция $\xi / \sin \xi$ монотонно возрастает на интервале $(\pi / 2, \pi)$, то неравенства (15.23) эквивалентны неравенствам

$$
\frac{\pi}{2}<\frac{\arcsin \sqrt{\gamma}}{\sqrt{1-\gamma}}<\frac{2(\pi-\arccos (\sqrt{3 \gamma} / 2))}{\sqrt{4-3 \gamma}}
$$


В результате получим, что (1.7) выполнено при $\gamma_{1}<\gamma<\alpha$, где $\alpha$ - решение уравнения

$$
\frac{\arcsin \sqrt{\alpha}}{\sqrt{1-\alpha}}=\frac{2(\pi-\arccos (\sqrt{3 \alpha} / 2))}{\sqrt{4-3 \alpha}} .
$$

Приближенные вычисления показывают, что $\alpha \sim 0.921485>\gamma_{2}$, следовательно, неравенство (1.7) выполнено при всех $\gamma \in\left(\gamma_{1}, \gamma_{2}\right)$.

2) Запишем условие $\mathbf{F}$ в виде

$$
\int U_{0}^{\prime \prime \prime}\left(s_{0}(x)\right) \varphi_{4 \lambda_{1}}(x) \varphi_{\lambda_{1}}^{2}(x) d x=\int \frac{d}{d x} U_{0}^{\prime \prime}\left(s_{0}(x)\right) \frac{\varphi_{4 \lambda_{1}}(x) \varphi_{\lambda_{1}}^{2}(x)}{s_{0}^{\prime}(x)} d x \neq 0 .
$$

Так как в силу (15.7)

$$
\frac{d}{d x} U_{0}^{\prime \prime}\left(s_{0}(x)\right)=(b+d) \delta(x-q)-(b+d) \delta(x+q),
$$

то (15.24) означает, что $\varphi_{4 \lambda_{1}}(q) \varphi_{\lambda_{1}}^{2}(q) \neq 0$. Из формулы $(15.9)$ следует, что $\varphi_{\lambda_{1}}(q)=A e^{-\alpha q} \neq 0$. Покажем, что равенство $\varphi_{4 \lambda_{1}}(q)=0$ имеет место только при одном значении $\gamma \in\left(\gamma_{1}, \gamma_{2}\right)$. Функция $\varphi_{4 \lambda_{1}}$ является нечетным решением уравнения

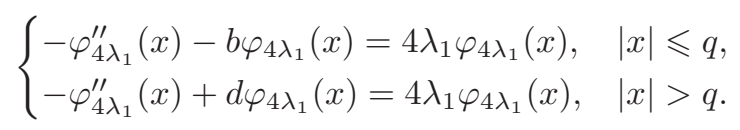

Поэтому $\varphi_{4 \lambda_{1}}(q)=\sin \beta q$, где $\beta=\sqrt{b+4 \lambda_{1}}>0$. Следовательно, равенство $\varphi_{4 \lambda_{1}}(q)=0$ выполнено только при условии $\beta q=k \pi, k \in \mathbb{N}$, что эквивалентно условию

$$
\sqrt{1+4 \gamma \lambda_{1}(\gamma)} \arcsin \sqrt{\gamma}=k \pi, \quad k \in \mathbb{N} .
$$

Подставляя сюда выражение для $\lambda_{1}(\gamma)$ из (15.18)-(15.19), получим

$$
\frac{\arcsin \sqrt{\gamma}}{\sqrt{1-\gamma}} \sqrt{4 \sin ^{2} \xi-3(1-\gamma)}=k \pi, \quad \frac{\xi^{2}}{\sin ^{2} \xi}=\frac{\arcsin ^{2} \sqrt{\gamma}}{1-\gamma} .
$$

Так как

$$
0<\frac{\arcsin \sqrt{\gamma}}{\sqrt{1-\gamma}} \sqrt{4 \sin ^{2} \xi-3(1-\gamma)}<2 \pi \quad \text { при } \gamma \in\left(\gamma_{1}, \gamma_{2}\right),
$$

то $k=1$. Обозначим $\theta=\arcsin \sqrt{\gamma}$. Тогда система (15.25) эквивалентна системе

$$
4 \xi^{2}-3 \theta^{2}=\pi^{2}, \quad \frac{\sin \xi}{\xi}=\frac{\cos \theta}{\theta} .
$$

Найдем решение графически. Выразим $\theta$ через $\xi$ из первого и второго уравнения и рассмотрим соответствующие функции $\theta_{1}(\xi)$ и $\theta_{2}(\xi)$. Функция $\theta_{1}(\xi):=$ $\sqrt{\left(4 \xi^{2}-\pi^{2}\right) / 3}$ возрастает на интервале $\left(\xi\left(\gamma_{1}\right), \xi\left(\gamma_{2}\right)\right)$. Кроме того,

$$
\theta_{1}^{\prime}(\xi)=\frac{1}{\sqrt{3}} \frac{4 \xi}{\sqrt{4 \xi^{2}-\pi^{2}}}>\frac{1}{\sqrt{3}} \frac{4(\pi / 2)}{\sqrt{4(3 \pi / 4)^{2}-\pi^{2}}}=\frac{4}{\sqrt{15}}>1, \quad \xi\left(\gamma_{1}\right)<\xi<\xi\left(\gamma_{2}\right)
$$




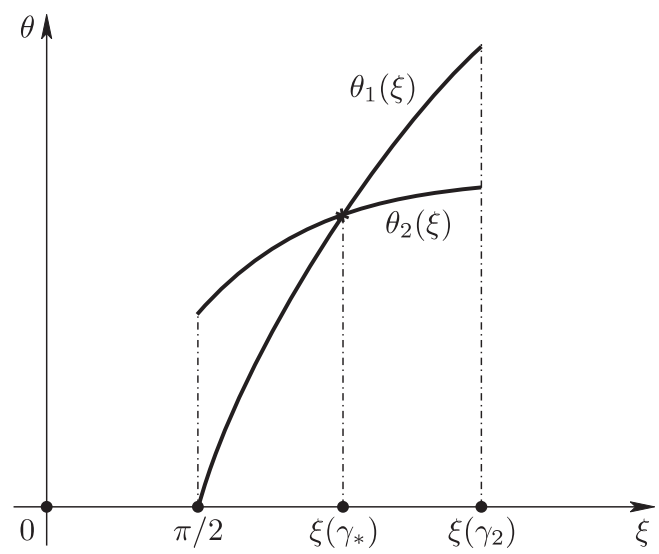

Рис. 8. Функции $\theta_{1}$ и $\theta_{2}$

так как $\xi\left(\gamma_{1}\right)=\pi / 2$ и $\xi\left(\gamma_{2}\right) \sim 2.3137<3 \pi / 4$. Вторая функция $\theta_{2}(\xi)$ неявно задается уравнением $(\sin \xi) / \xi=(\cos \theta) / \theta$. Найдем ее производную:

$$
\theta_{2}^{\prime}(\xi)=\frac{\sin \xi-\xi \cos \xi}{\xi^{2}} \frac{\theta^{2}}{\cos \theta+\theta \sin \theta}>0, \quad \frac{\pi}{2}<\xi<\xi\left(\gamma_{2}\right) .
$$

Кроме того, из (15.26) следует, что

$$
\theta_{2}^{\prime}(\xi)=\frac{\theta}{\xi} \frac{(\sin \xi) / \xi-\cos \xi}{(\cos \theta) / \theta+\sin \theta}<\frac{(\sin \xi) / \xi-\cos \xi}{(\sin \xi) / \xi+\sin \theta}<1, \quad \frac{\pi}{2}<\xi<\xi\left(\gamma_{2}\right),
$$

так как $|\cos \xi|<\left|\cos \xi\left(\gamma_{2}\right)\right|<\sqrt{2} / 2$ и $\sin \theta=\sqrt{\gamma}>\sqrt{\gamma}{ }_{1}>\sqrt{2} / 2$ в силу (15.17). Далее,

$$
\theta_{2}\left(\frac{\pi}{2}\right)>\theta_{1}\left(\frac{\pi}{2}\right)=0, \quad \theta_{2}\left(\xi\left(\gamma_{2}\right)\right) \sim 1.1843<\theta_{1}\left(\xi\left(\gamma_{2}\right)\right) \sim 1.9616 .
$$

Из (15.27)-(15.30) следует, что $\theta_{1}(\xi)=\theta_{2}(\xi)$ только для одного значения $\xi\left(\gamma_{*}\right) \in$ $\left(\pi / 2, \xi\left(\gamma_{2}\right)\right)$ (см. рис. 8). Решая численно систему (15.26), получим $\gamma_{*} \sim 0.7925$. Таким образом, условие $\mathbf{F}$ справедливо для всех $\gamma \in\left(\gamma_{1}, \gamma_{2}\right)$, кроме одной точки $\gamma_{*}$.

Итак: при $\gamma \in\left(\gamma_{1}, \gamma_{*}\right) \cup\left(\gamma_{*}, \gamma_{2}\right)$ выполнены все условия второй главы, за исключением условия гладкости потенциала в точках $\pm \gamma$.

\section{6. Гладкие аппроксимации}

Построим аппроксимации потенциала $U_{0}$ гладкими потенциалами. Рассмотрим четную функцию $h(\psi) \in C_{0}^{\infty}(\mathbb{R})$ такую, что $h(\psi) \geqslant 0, \operatorname{supp} h \subset[-1,1]$ и $\int h(\psi) d \psi=1$. Для любого $\varepsilon \in(0, \gamma)$ положим

$$
\widetilde{U}_{\varepsilon}(\psi):=\frac{1}{\varepsilon} \int h\left(\frac{\psi-\psi^{\prime}}{\varepsilon}\right) U_{0}\left(\psi^{\prime}\right) d \psi^{\prime}
$$


Функция $\widetilde{U}_{\varepsilon}(\psi) \geqslant 0$ является гладкой четной функцией, симметричной относительно точек $\psi= \pm 1$ в некоторой окрестности этих точек. Кроме того,

$$
\widetilde{U}_{\varepsilon}(\psi)-U_{0}(\psi)= \begin{cases}\mu_{\varepsilon}>0, & |\psi| \geqslant \gamma+\varepsilon \\ -\nu_{\varepsilon}<0, & |\psi| \leqslant \gamma-\varepsilon\end{cases}
$$

где $\mu_{\varepsilon}, \nu_{\varepsilon}=\mathscr{O}\left(\varepsilon^{2}\right)$. Положим $U_{\varepsilon}(\psi)=\widetilde{U}_{\varepsilon}(\psi)-\mu_{\varepsilon}$. Тогда

$$
U_{\varepsilon}(\psi)= \begin{cases}U_{0}(\psi), & |\psi| \geqslant \gamma+\varepsilon \\ U_{0}(\psi)-\mu_{\varepsilon}-\nu_{\varepsilon}, & |\psi| \leqslant \gamma-\varepsilon\end{cases}
$$

Очевидно, что $\sup \left|U_{\varepsilon}(\psi)-U_{0}(\psi)\right| \leqslant C \varepsilon$ с некоторой константой $C>0$. Кроме того, $U_{\varepsilon}^{\prime \prime \prime}(\psi) \leqslant 0$ при $\psi \leqslant 0$. Соответствующий кинк является нечетным решением уравнения $s_{\varepsilon}^{\prime \prime}(x)-U_{\varepsilon}^{\prime}\left(s_{\varepsilon}(x)\right)=0$. Проинтегрировав это уравнение, получим

$$
\int_{0}^{s_{\varepsilon}(x)} \frac{d s}{\sqrt{2 U_{\varepsilon}\left(s_{\varepsilon}\right)}}=x, \quad x \in \mathbb{R} .
$$

Поэтому $s_{\varepsilon}(x)$ является монотонно возрастающей функцией, $s_{\varepsilon}(x) \rightarrow \pm 1$ при $x \rightarrow \pm \infty$. Из (16.2) следует, что $\left|s_{\varepsilon}(x)-s_{0}(x)\right| \leqslant C_{1} \varepsilon$. Значит, ||$s_{\varepsilon}(x)|-\gamma| \geqslant \varepsilon$ при ||$x|-q| \geqslant \delta$, где

$$
\delta \rightarrow 0, \quad \varepsilon \rightarrow 0
$$

Следовательно, $W_{\varepsilon}(x):=U_{\varepsilon}^{\prime \prime}\left(s_{\varepsilon}(x)\right)=W_{0}(x)$ при ||$x|-q| \geqslant \delta$ и

$$
\left|W_{\varepsilon}(x)-W_{0}(x)\right| \leqslant b+d, \quad x \in \mathbb{R} .
$$

Обозначим $w_{\varepsilon}(x)=W_{\varepsilon}(x)-W_{0}(x)$. Из (16.4) и (16.5) следует, что

$$
\left\|w_{\varepsilon}\right\|_{L^{2}(\mathbb{R})} \rightarrow 0, \quad \varepsilon \rightarrow 0 .
$$

Очевидно, что для потенциалов $U_{\varepsilon}$ выполнены условия U1 и U2. Проверим выполнение условия U3. Согласно теореме 7.2 из [31], условие отсутствия резонанса в концевой точке непрерывного спектра эквивалентно ограниченности резольвенты в этой точке. Резольвенты $R_{0}(\omega)$ и $R_{\varepsilon}(\omega)$ операторов $H_{0}$ и $H_{\varepsilon}:=-d^{2} / d x^{2}+W_{\varepsilon}(x)$ связаны соотношением

$$
R_{\varepsilon}(\omega)=R_{0}(\omega)\left(1+w_{\varepsilon} R_{0}(\omega)\right)^{-1}
$$

из которого в силу (16.6) следует, что для достаточно малых $\varepsilon>0$ и всех $\gamma \in(0,1) \backslash\left\{\gamma_{k}\right\}$ потенциалы $U_{\varepsilon}(\psi)$ удовлетворяют условию $\mathbf{U} \mathbf{3}$.

Из (16.6) и (16.7) следует также, что собственные значения оператора $H_{\varepsilon}$ сходятся к собственным значениям оператора $H_{0}$ при $\varepsilon \rightarrow 0$. Следовательно,

i) при всех $\gamma \in\left(0, \gamma_{1}\right)$ и достаточно малых $\varepsilon$ оператор $H_{\varepsilon}$ имеет только одно нулевое собственное значение, т. е. потенциалы $U_{\varepsilon}$ удовлетворяют спектральному условию D1; 
ii) при $\gamma \in\left(\gamma_{1}, \gamma_{2}\right)$ и достаточно малых $\varepsilon$ оператор $H_{\varepsilon}$ имеет два собственных значения $\lambda_{0}=0$ и $0<\lambda_{1}(\varepsilon)<d$, причем $4 \lambda_{1}(\varepsilon)>d$, т. е. потенциалы $U_{\varepsilon}$ удовлетворяют спектральному условию D2.

Легко проверить, что при малых $\varepsilon$ и всех $\gamma \in\left(\gamma_{1}, \gamma_{2}\right) \backslash\left\{\gamma_{*}\right\}$ условие $\mathbf{F}$ тоже выполнено.

\section{Список литературы}

[1] S. Agmon, "Spectral properties of Schrödinger operators and scattering theory", Ann. Scuola Norm. Sup. Pisa Cl. Sci. (4), 2:2 (1975), 151-218.

[2] N. Boussaid, "Stable directions for small nonlinear Dirac standing waves", Comm. Math. Phys., 268:3 (2006), 757-817.

[3] N. Boussaid, S. Cuccagna, "On stability of standing waves of nonlinear Dirac equations", Comm. Partial Differential Equations, 37:6 (2012), 1001-1056; arXiv: 1103.4452v3.

[4] V.S. Buslaev, A. I. Komech, E. A. Kopylova, D. Stuart, "On asymptotic stability of solitary waves in Schrödinger equation coupled to nonlinear oscillator", Comm. Partial Differential Equations, 33:4-6 (2008), 669-705.

[5] В.С. Буслаев, Г. С. Перельман, "Рассеяние лля нелинейного уравнения Шрёдингера: состояния, близкие к солитону", Алгебра и анализ, 4:6 (1992), 63-102; англ. пер.: V.S. Buslaev, G. S. Perelman, "Scattering for the nonlinear Schrödinger equation: states close to a soliton", St. Petersburg Math. J., 4:6 (1993), 1111-1142.

[6] V.S. Buslaev, G.S. Perelman, "On the stability of solitary waves for nonlinear Schrödinger equations", Nonlinear evolution equations, Amer. Math. Soc. Transl. Ser. 2, 164, Amer. Math. Soc., Providence, RI, 1995, 75-98.

[7] V.S. Buslaev, C. Sulem, "On asymptotic stability of solitary waves for nonlinear Schrödinger equations", Ann. Inst. H. Poincaré Anal. Non Linéaire, 20:3 (2003), 419-475.

[8] S. Cuccagna, "Stabilization of solutions to nonlinear Schrödinger equations", Comm. Pure Appl. Math., 54:9 (2001), 1110-1145.

[9] S. Cuccagna, "On asymptotic stability in 3D of kinks for the $\phi^{4}$ model", Trans. Amer. Math. Soc., 360:5 (2008), 2581-2614.

[10] W. Eckhaus, A. van Harten, The inverse scattering transformation and the theory of solitons. An introduction, North-Holland Math. Stud., 50, North-Holland Publishing Co., Amsterdam-New York, 1981, xi+222 pp.

[11] A. S. Fokas, V. E. Zakharov (eds.), Important developments in soliton theory, Springer Ser. Nonlinear Dynam., Springer-Verlag, Berlin, 1993, x+559 pp.

[12] C.S. Gardner, J. M. Greene, M.D. Kruskal, R. M. Miura, "Method for solving the Korteweg-de Vries equation", Phys. Rev. Lett., 19 (1967), 1095-1097.

[13] W. Heisenberg, "Der derzeitige Stand der nichtlinearen Spinortheorie der Elementarteilchen", Acta Phys. Austriaca, 14 (1961), 328-339.

[14] W. Heisenberg, Introduction to the unified field theory of elementary particles, Interscience Publishers, London-New York-Sydney, 1966, ix+177 pp.

[15] V. Imaykin, A. Komech, H. Spohn, "Scattering asymptotics for a charged particle coupled to the Maxwell field", J. Math. Phys., 52:4 (2011), 042701, 33 pp.

[16] V. Imaikin, A. Komech, B. Vainberg, "On scattering of solitons for the Klein-Gordon equation coupled to a particle", Comm. Math. Phys., 268:2 (2006), 321-367.

[17] E. Kirr, A. Zarnesku, "On the asymptotic stability of bound states in 2D cubic Schrödinger equation", Comm. Math. Phys., 272:2 (2007), 443-468. 
[18] A. Komech, E. Kopylova, "Scattering of solitons for Schrödinger equation coupled to a particle", Russ. J. Math. Phys., 13:2 (2006), 158-187; arXiv: math/0609649.

[19] A.I. Komech, E. A. Kopylova, "Weighted energy decay for 1D Klein-Gordon equation", Comm. Partial Differential Equations, 35:2 (2010), 353-374.

[20] A. I. Komech, E. A. Kopylova, H. Spohn, "Scattering of solitons for Dirac equation coupled to a particle", J. Math. Anal. Appl., 383:2 (2011), 265-290; arXiv: 1012.3109.

[21] A. Komech, E. Kopylova, D. Stuart, "On asymptotic stability of solitons in a nonlinear Schrödinger equation", Commun. Pure Appl. Anal., 11:3 (2012), 1063-1079; arXiv: 0807.1878.

[22] A.I. Komech, N. J. Mauser, A. P. Vinnichenko, "Attraction to solitons in relativistic nonlinear wave equations", Russ. J. Math. Phys., 11:3 (2004), 289-307.

[23] E. Kopylova, "On the asymptotic stability of solitary waves in the discrete Schrödinger equation coupled to a nonlinear oscillator", Nonlinear Anal., 71:7-8 (2009), 3031-3046; arXiv: 0805.3403.

[24] Е.А. Копылова, “Дисперсионные оценки для уравнений Шрёдингера и КлейнаГордона", УМН, 65:1(391) (2010), 97-144; англ. пер.: Е. A. Kopylova, "Dispersive estimates for the Schrödinger and Klein-Gordon equation", Russian Math. Surveys, 65:1 (2010), 95-142.

[25] E. A. Kopylova, "On asymptotic stability of solitary waves in discrete Klein-Gordon equation coupled to nonlinear oscillator", Appl. Anal., 89:9 (2010), 1467-1492.

[26] E. Kopylova, "On long-time decay for modified Klein-Gordon equation", Commun. Math. Anal., Conference 03 (2011), 137-152; arXiv: 1009.2649.

[27] E. A. Kopylova, A. I. Komech, "On asymptotic stability of moving kink for relativistic Ginzburg-Landau equation", Comm. Math. Phys., 302:1 (2011), 225-252; arXiv: 0910.5538.

[28] E. Kopylova, A. I. Komech, "On asymptotic stability of kink for relativistic Ginzburg-Landau equation", Arch. Ration. Mech. Anal., 202:1 (2011), 213-245; arXiv: 0910.5539.

[29] Ж.-Л. Лионс, Некоторые методы решения нелинейных краевых задач, Мир, М., 1972, 587 c.; пер. с фр.: J.-L. Lions, Quelques méthodes de résolution des problèmes aux limites non linéaires, Dunod, Paris; Gauthier-Villars, Paris, 1969, xx+554 pp.

[30] J. R. Miller, M. I. Weinstein, "Asymptotic stability of solitary waves for the regularized long-wave equation", Comm. Pure Appl. Math., 49:4 (1996), 399-441.

[31] M. Murata, "Asymptotic expansions in time for solutions of Schrödinger-type equations", J. Funct. Anal., 49:1 (1982), 10-56.

[32] R. L. Pego, M. I. Weinstein, "Asymptotic stability of solitary waves", Comm. Math. Phys., 164:2 (1994), 305-349.

[33] D. E. Pelinovsky, A. A. Stefanov, "Asymptotic stability of small gap solitons in nonlinear Dirac equations", J. Math. Phys., 53:7 (2012), 073705, 27 pp.; arXiv: 1008.4514.

[34] C.-A. Pillet, C. E. Wayne, "Invariant manifolds for a class of dispersive, Hamiltonian, partial differential equations", J. Differential Equations, 141:2 (1997), 310-326.

[35] M. Reed, Abstract non-linear wave equations, Lecture Notes in Math., 507, Springer-Verlag, Berlin-New York, 1976, vi+128 pp.

[36] М. Рид, Б. Саймон, Методъ современной математической физики, т. 3: Теоpия рассеяния, Мир, М., 1982, 445 с.; пер. с англ.: М. Reed, B. Simon, Methods of modern mathematical physics, v. III: Scattering theory, Academic Press, New York-London, 1979, xv+463 pp.

[37] I. Rodnianski, W. Schlag, A. Soffer, "Dispersive analysis of charge transfer models", Comm. Pure Appl. Math., 58:2 (2005), 149-216.

[38] A. Soffer, M. I. Weinstein, "Multichannel nonlinear scattering for nonintegrable equations", Comm. Math. Phys., 133:1 (1990), 119-146. 
[39] A. Soffer, M. I. Weinstein, "Multichannel nonlinear scattering for nonintegrable equations. II. The case of anisotropic potentials and data", J. Differential Equations, 98:2 (1992), 376-390.

[40] A. Soffer, M. I. Weinstein, "Resonances, radiation damping and instability in Hamiltonian nonlinear wave equations", Invent. Math., 136:1 (1999), 9-74.

[41] W. A. Strauss, "Nonlinear invariant wave equations", Invariant wave equations, Proceedings of the "Ettore Majorana" International School of Mathematical Physics (Erice, 1977), Lecture Notes in Phys., 73, Springer, Berlin-New York, 1978, 197-249.

[42] Tai-Peng Tsai, Horng-Tzer Yau, "Asymptotic dynamics of nonlinear Schrödinger equations: resonance-dominated and dispersion-dominated solutions", Comm. Pure Appl. Math., 55:2 (2002), 153-216.

[43] Tai-Peng Tsai, "Asymptotic dynamics of nonlinear Schrödinger equations with many bound states", J. Differential Equations, 192:1 (2003), 225-282.

[44] M. Weinstein, "Modulational stability of ground states of nonlinear Schrödinger equations", SIAM J. Math. Anal., 16:3 (1985), 472-491.

[45] N. J. Zabusky, M. D. Kruskal, "Interaction of "solitons" in a collisionless plasma and the recurrence of initial states", Phys. Rev. Lett., 15 (1965), 240-243.

Е. А. Копылова (Е. А. Kоруlova)

Институт проблем передачи информации РАН;

University of Vienna, Vienna, Austria

E-mail: elena.kopylova@univie.ac.at
Поступила в редакцию

06.02 .2013 